\title{
Cryo-EM Structures of Respiratory bc1-cbb3 type ClII2CIV Supercomplex and Electronic Communication Between the Complexes
}

\section{Stefan Steimle}

University of Pennsylvania

\section{Trevor Van Eeuwen}

University of Pennsylvania https://orcid.org/0000-0003-0549-1702

Yavuz Ozturk

University of Pennsylvania

Hee Jong Kim

University of Pennsylvania https://orcid.org/0000-0002-0823-5301

Merav Braitbard

Hebrew University

Nur Selamoglu

University of Pennsylvania

Benjamin Garcia

University of Pennsylvania

Dina Schneitman-Duhovny

Hebrew University

Kenji Murakami

UPenn https://orcid.org/0000-0002-2017-5575

Fevzi Daldal ( $\square$ fdaldal@sas.upenn.edu )

University of Pennsylvania

\section{Article}

Keywords: Cryo-EM Structures, bc1, aa3-type CIV, cyt cy

Posted Date: July 29th, 2020

DOI: https://doi.org/10.21203/rs.3.rs-48203/v1

License: (1) (1) This work is licensed under a Creative Commons Attribution 4.0 International License.

Read Full License 
Version of Record: A version of this preprint was published at Nature Communications on February 10th, 2021. See the published version at https://doi.org/10.1038/s41467-021-21051-4. 
*Corresponding authors: Fevzi Daldal: fdaldal@sas.upenn.edu

${ }^{1}$ Department of Biology, University of Pennsylvania, Philadelphia, PA, 19104; ${ }^{2}$ Biochemistry and Molecular Biophysics Graduate Group, Perelman School of Medicine, University of Pennsylvania, Philadelphia, PA 19104; \#Institute of Biochemistry and Molecular Biology, Faculty of Medicine, Albert-Ludwigs University of Freiburg, 79104 Freiburg, Germany; ${ }^{3}$ School of Computer Science and Engineering, Institute of Life Sciences, The Hebrew University of Jerusalem, Jerusalem, 9190401, Israel and ${ }^{4}$ Department of Biochemistry and Biophysics, Perelman School of Medicine, University of Pennsylvania, Philadelphia, PA 19104

Running title: Bacterial respiratory cytochrome $b c_{1}-c b b_{3}$ supercomplex

Key words: cytochrome $b c_{1}$ or Complex III; Cytochrome $c b b_{3}$ oxidase or Complex IV; respiratory supercomplex; electron carrier cytochrome $c$ : membrane-anchored cytochrome $c_{y}$; soluble cytochrome $c_{2}$; Rhodobacter capsulatus; respiratory electron transport chain

\author{
Phone: +1 215 898-4394 \\ Kenji Murakami: kenjim@pennmedicine.upenn.edu \\ Phone: +1 215 573-1125
}




\section{Abbreviations}

$46 \mathrm{Q}$, quinone; $\mathrm{QH}_{2}$, Quinol or hydroquinone; Complex III, $\mathrm{CIII}_{2}$ or cytochrome $b c_{1}$, ubiquinol47 cytochrome $c$ oxidoreductase; Complex IV or CIV, $c b b_{3}$-type cytochrome $c$ oxidase; cyt, cytochrome;

48 cyt $c_{2}$, cytochrome $c_{2}$, soluble cytochrome $c$; cyt $c_{\mathrm{y}}$, cytochrome $c_{\mathrm{y}}$, membrane-anchored cytochrome

$49 \quad c$; cyt S- $c_{\mathrm{y}}$, cytochrome $c_{\mathrm{y}}$ without its membrane anchor; SC, super-complex; MS, mass spectrometry;

50 XL-MS, cross-linking mass spectrometry; XL, cross-links; TMBZ, 3,3' ,5,5'-tetramethyl-benzidine;

$51 \mathrm{DBH}_{2}$, 2,3-dimethoxy-5-methyl-6-decyl-1,4-benzoquinone; FeS, Rieske iron-sulfur protein; FeS-ED,

52 membrane-extrinsic domain of FeS protein; b position, location of the [2Fe-2S] cluster near heme $b_{\mathrm{L}}$;

$53 \mathrm{c}$ position, location of the $[2 \mathrm{Fe}-2 \mathrm{~S}]$ cluster near heme $c_{1}$; cryo-EM, cryogenic electron microscopy;

54 BN-PAGE and SDS-PAGE, blue native and sodium dodecylsulfate polyacrylamide gel

55 electrophoresis; C-ter, C-terminus; N-ter, N-terminus; SEC, size exclusion chromatography; TMH,

56 transmembrane helix; DSBU, disuccinimidyl dibutyric urea; DMTMM, 4-(4,6-dimethoxy-1,3,5-

57 triazin-2-yl)-4-methyl-morpholinium chloride; heme-Fe, heme-iron; $\mathrm{E}_{\mathrm{m}}$, redox midpoint potential;

58 heme $c_{\mathrm{p} 1}, \mathrm{~N}$-ter $c$-type heme 1 of CcoP; heme $c_{\mathrm{p} 2}$, C-ter $c$-type heme 2 of CcoP; heme $c_{\mathrm{o}}, c$-type heme

59 of CcoO; RMSD, root-mean-square deviation; DDM, n-dodecyl $\beta$-D-maltoside. 


\section{Abstract}

61 Respiratory electron transport complexes are organized as individual entities or combined as large

62 super-complexes (SC). The Gram-negative bacteria deploy a mitochondrial-like cytochrome (cyt)

$63 b c_{1}\left(\right.$ Complex III, $\left.\mathrm{CIII}_{2}\right)$, and may have specific $c b b_{3}$-type cyt $c$ oxidases (Complex IV, CIV) instead

64 of the canonical $a a_{3}$-type CIV. Electron transfer between these complexes is mediated by soluble $\left(c_{2}\right)$

65 and membrane-anchored $\left(c_{\mathrm{y}}\right)$ cyts. Here, we report the first structure of a $b c_{1}-c b b_{3}$ type SC $\left(\mathrm{CIII}{ }_{2} \mathrm{CIV}\right.$,

$665.2 \AA$ resolution) and three conformers of native $\mathrm{CIII}_{2}$ (3.3 $\AA$ resolution) of functional relevance. The

67 SC contains all catalytic subunits and cofactors as well as two extra transmembrane helices attributed

68 to cyt $c_{\mathrm{y}}$ and the assembly factor $\mathrm{CcoH}$. The cyt $c_{\mathrm{y}}$ is integral to $\mathrm{SC}$, its cyt domain is mobile and

69 conveys electrons to CIV differently than cyt $c_{2}$. For the first time, this work establishes the structural

70 characteristics of membrane-confined and membrane-external electron transport pathways of SCs in

71 Gram-negative bacteria. 


\section{Introduction}

73 Respiratory chains couple exergonic electron transport from nutrients to the terminal acceptor oxygen

$74\left(\mathrm{O}_{2}\right)$, and generate a proton motive force used for ATP synthesis. Complex I (NADH dehydrogenase)

75 and Complex II (succinate dehydrogenase) are the entry points into the chain of reducing equivalents,

76 and reduce quinone $(\mathrm{Q})$ to hydroquinone $\left(\mathrm{QH}_{2}\right)$. Complex III (cytochrome (cyt) $b c_{1}$ or $\mathrm{CIII}_{2}$ ) oxidizes

$77 \mathrm{QH}_{2}$ to reduce cyt $c$, which is then oxidized by Complex IV (cyt $c$ oxidase or CIV) converting oxygen

78 to water ${ }^{1}$ (Fig. 1A).

79 Respiratory complexes are evolutionarily conserved, but bacterial enzymes are structurally

80 simpler, consisting mainly of the catalytic subunits. $\mathrm{CIII}_{2}$ is a dimer with each monomer comprised

81 of three subunits: the Rieske FeS (FeS) protein with a [2Fe-2S] cluster, cyt $b$ with hemes $b_{\mathrm{H}}$ and $b_{\mathrm{L}}$,

82 and cyt $c_{1}$ with heme $c_{1}$ cofactors (Fig. 1A,B). The FeS protein external domain (FeS-ED) is mobile

83 between the $\mathbf{b}$ (close to heme $b_{\mathrm{L}}$ ) and $\mathbf{c}$ (close to heme $c_{1}$ ) positions ${ }^{2,3}$. Some bacterial species such as

84 Rhodobacter capsulatus ${ }^{4}$ and pathogens like Helicobacter pylori, Campylobacter jejuni ${ }^{5}$ and

85 Neisseria ${ }^{6}$ contain only a high oxygen affinity $c b b_{3}$-type CIV to support their micro-aerophilic

86 growth. The $c b b_{3}$-type CIV is a monomer comprised of four subunits: CcoN with heme $b$ and heme

$87 b_{3}-\mathrm{Cu}$ binuclear center, $\mathrm{CcoO}$ with heme $c_{\mathrm{o}}, \mathrm{CcoQ}$, and $\mathrm{CcoP}$ with hemes $c_{\mathrm{p} 1}$ and $c_{\mathrm{p} 2}$ cofactors ${ }^{7}$ (Fig.

$88 \mathbf{1 A}, \mathbf{B})$.

89 Many Gram-negative bacteria contain both soluble and membrane-anchored (via transmembrane

90 domains or fatty acids) cyts $c$ as electron carriers (e.g., Rhodobacter capsulatus cyt $c_{\mathrm{y}}{ }^{8}$, Paracoccus

91 denitrificans cyt $c_{552}{ }^{9}$, and Helicobacterium gestii cyt $\left.c_{553}{ }^{10}\right)$. Conversely, Gram-positive bacteria

92 have no freely diffusing electron carrier, but contain cyt $c$ domains fused to their $\mathrm{CIII}_{2}$ such as in

93 Mycobacterium smegmatis ${ }^{11}$, or CIV such as in Bacillus subtilis ${ }^{12}$. In $R$. capsulatus, both the

94 diffusible cyt $c_{2}$ and the membrane-anchored cyt $c_{\mathrm{y}}$ are electron carriers from $\mathrm{CIII}_{2}$ to $\mathrm{CIV}$ in

95 respiration ${ }^{13}$. 
96 In recent years, the co-occurrence of individual complexes together with multi-enzyme super97 complexes (SCs) in energy-transducing membranes has become evident ${ }^{14,15}$. However, the role of 98 this heterogeneity is debated ${ }^{16,17}$. SCs may enhance catalytic efficiency through substrate/product 99 channeling, or minimize production of harmful reactive oxygen species to decrease cellular distress $100{ }^{18}$. The structures of mitochondrial SCs, such as $\mathrm{CICIII}_{2} \mathrm{CIV}$ or its smaller variants $\mathrm{CICIII}_{2}$ and $101 \mathrm{CIII}_{2} \mathrm{CIV}^{19}$ are well-known ${ }^{20,21}$. Some bacterial SCs (e.g., $P$. denitrificans ${ }^{22}$ or $C$. glutamicum ${ }^{23}$ ) 102 have also been characterized biochemically, but only the structure of the Gram-positive M. smegmatis $103 \mathrm{SC}\left(\mathrm{CIII}_{2} \mathrm{CIV}_{2}\right)$ is known ${ }^{24,25}$.

104 As of yet, no respiratory SC structure has been determined for Gram-negative bacteria, the 105 evolutionary precursors of mitochondria. Moreover, SCs containing $c b b_{3}$-type ancient forms of CIV 106 with primordial respiratory features remain unknown ${ }^{26}$. Such structural studies have been hampered 107 due to unstable interactions between $\mathrm{CIII}_{2}$ and $\mathrm{CIV}$, hence their trace amounts in nature. We have 108 overcome this hurdle using a genetic approach, yielding large amounts of SCs from the Gram109 negative facultative phototroph $R$. capsulatus. Here, we report the first cryo-EM structure of a $b c_{1^{-}}$

$110 c b b_{3}$ type $\mathrm{SC}\left(\mathrm{CIII}_{2} \mathrm{CIV}\right.$, at $5.2 \AA$ resolution), as well as three structural conformers of native $\mathrm{CIII}_{2}$ (at $1113.3-4.2 \AA$ resolution). Moreover, we show that the membrane-bound cyt $c_{\mathrm{y}}$ and the diffusible cyt $c_{2}$ 112 interact differently with CIV for electron transfer. For the first time, this work establishes the 113 structural features of $\mathrm{CIII}_{2} \mathrm{CIV}$ and its distinct respiratory electron transport pathways (membrane114 confined and membrane-peripheral) in Gram-negative bacteria. 


\section{Results}

116 Isolation, and characterization of functional fused SCs. Earlier studies have indicated that in $R$.

117 capsulatus $\mathrm{CIII}_{2}, \mathrm{CIV}$, and cyt $c_{\mathrm{y}}$ are in close proximity to each other ${ }^{27}$. Analyses of membranes from

118 this species indicated barely detectable entities around $\sim 450 \mathrm{kDa} \mathrm{M}_{\mathrm{r}}$, larger than the masses of CIV

119 monomers or $\mathrm{CIII}_{2}$ dimers (Fig. S1A,B). However, these entities were of low abundance and highly

120 unstable, rendering their study difficult.

121 During the assembly processes of $\mathrm{CIII}_{2}$ and CIV, cyt $c_{1}$ interacts with cyt $b$ to form a cyt $b$ - $c_{1}$ 122 subcomplex ${ }^{28}$, and CcoP associates with CcoNOQ subcomplex to yield an active CIV ${ }^{29}$. We surmised

123 that translationally fusing the $\mathrm{C}$-terminus (C-ter) of cyt $c_{1}$ to the $\mathrm{N}$-terminus (N-ter) of $\mathrm{CcoP}$ (on the 124 inner ( $n$ ) side of the membrane) as a cyt $c_{1}$-CcoP fusion might produce a stable bipartite $b c_{1}-c b b_{3}$ type 125 SC (left panels of Fig. 1C,D). Also, adding the 69-residue linker (L) and the 100-residue cyt domain 126 of $c_{\mathrm{y}}$ to the C-ter of cyt $c_{1}-\mathrm{CcoP}$ (on the outer $(p)$ side of the membrane) as a cyt $c_{1}-\mathrm{CcoP}-c_{\mathrm{y}}$ protein 127 might yield a tripartite SC with its attached electron carrier (right panels of Fig. 1C,D). This approach 128 (Supplemental Information, Methods) was successful, and yielded constructs (Fig. S1B and Table 129 S1) that complemented a mutant lacking $\mathrm{CIII}_{2}$ and $\mathrm{CIV}$ for photosynthesis-proficiency (i.e., $\mathrm{CIII}_{2}$ 130 activity) and CIV activity (Fig. S1C).

131 The His-tagged bipartite and Flag-tagged tripartite SCs were purified by tag-affinity and size 132 exclusion chromatography (SEC) (SI, Methods) (Fig. S1D,E). Analyses of isolated proteins showed 133 that they contained large entities of $\mathrm{M}_{\mathrm{r}} \sim 450 \mathrm{kDa}$ range (Fig. S1D,E fractions A-1 and B-1 in insets) 134 that had the cyt $c_{1}-\mathrm{CcoP}(\sim 65 \mathrm{kDa})$ or cyt $c_{1}-\mathrm{CcoP}-c_{\mathrm{y}}$ fusions $(\sim 80 \mathrm{kDa})(\mathbf{F i g} . \mathbf{S 1 F})$, and all protein 135 bands were identified by mass spectrometry (MS) as the subunits of $\mathrm{CIII}_{2}$ and CIV (Table S2). The 136 fusion proteins contained covalently-attached heme cofactor(s) as revealed by a $c$-type cyt specific 137 staining (Fig. S1G). CcoQ $\left(\mathrm{M}_{\mathrm{r}} \sim 7 \mathrm{kDa}\right)$ of CIV was absent in both SC preparations. 
Purified SCs were characterized for their cyts $b$ and $c$ contents. The optical redox difference

139 spectra were distinct from those of $\mathrm{CIII}_{2}{ }^{30}$ or CIV ${ }^{31}$, and the tripartite SC contained more $c$-type cyt

140 than the bipartite SC, due to the additional cyt domain of $c_{\mathrm{y}}$ (Fig. S1H). Both SC preparations were

141 active, exhibiting $\mathrm{QH}_{2}$ :cyt $c$ reductase (specific to $\mathrm{CIII}_{2}$ ) and cyt $c$ reductase (specific to CIV)

142 activities (Fig. S1I,J). Moreover, the tripartite $\mathrm{SC}$ also had $\mathrm{QH}_{2}: \mathrm{O}_{2}$ reductase (coupled CIII ${ }_{2}+\mathrm{CIV}$ )

143 activity without addition of horse heart cyt $c$ or $R$. capsulatus cyt $c_{2}$ (Fig.S1K). Thus, the cyt domain

144 of $c_{\mathrm{y}}$ fused to cyt $c_{1}$-CcoP transferred electrons from $\mathrm{CIII}_{2}$ to $\mathrm{CIV}$.

146 Structures of the tripartite SCs. Cryo-EM analysis of the tripartite SC preparations (Fig. S1E,

147 fraction B-1) showed that the initial 3D classes were mainly of two different sizes (Fig. S2, Box 1,

148 left). The size $(\sim 180 \AA$ length) and shape of the smaller particles suggested that these may correspond

149 to a dimeric $\mathrm{CIII}_{2}$ associated with a single CIV. Focused classification and processing of the subclass

150 containing $\sim 62,000$ particles with the highest initial resolution and best discernable features, led to a

151 tripartite $\mathrm{CIII}_{2} \mathrm{CIV}$ map (SC-1A, EMD-22228) at $6.1 \AA$ resolution (Fig. S2A, SI Methods), while

152 another dataset yielded a lower resolution map (SC-1B, EMD-22230) at 7.2 ̊ (Fig. S2B) (Table S3).

153 The larger particles ( 250Å length, Fig. S2, Box 1, left) represented a dimeric $\mathrm{CIII}_{2}$ flanked by two

$154 \mathrm{CIV}$, as expected based on two $c_{1}-\mathrm{CcoP}-c_{\mathrm{y}}$ subunits per $\mathrm{CIII}_{2}$. However these particles were rare

$155(\sim 5,000)$ and their map (SC-1C) could not be refined beyond $\sim 10 \AA$ resolution $($ Fig. S2C).

156 A homology model of $R$. capsulatus $c b b_{3}$-type CIV was built using the highly homologous $P$.

157 stutzeri structure (PDB: 3 MK7; $3.2 \AA ̊$ resolution) as a template (SI, Methods, Table S3). The available

$158 \mathrm{CIII}_{2}$ model (PDB: 1ZRT; 3.5Å resolution) was further refined (PDB: 6XI0; 3.3Å resolution) using 159 our cryo-EM data (Table S3). These models were fitted as rigid bodies into the maps SC1-A and SC-

160 1B (Fig. S3A), with correlation coefficients $\mathrm{CC}_{\mathrm{box}}$ of 0.75 and $\mathrm{CC}_{\mathrm{box}}$ of 0.71 , respectively (Table S3).

161 The $[2 \mathrm{Fe}-2 \mathrm{~S}]$ clusters of the FeS proteins of $\mathrm{CIII}_{2}$ could be recognized closer to $\mathrm{b}$ (heme $b_{\mathrm{L}}$ ) than to c 
162 (heme $c_{1}$ ) positions, but had lower occupancy and resolution likely due to conformational

163 heterogeneity. The heterogeneity of the FeS-ED of $\mathrm{CIII}_{2}$ in monomer B (away from CIV) was more 164 pronounced than that in monomer A (adjacent to CIV) (Fig. S3B). Lower resolutions of the CIII ${ }_{2} \mathrm{FeS}_{-}$ 165 ED portions were anticipated due to their mobility ${ }^{2,3}$.

166 Superimposition of the $\mathrm{CIII}_{2}$ portions of SC-1A and SC-1B maps showed that CIV was in different 167 orientations in different maps (Fig. S3C). The two extreme locations of CIV with respect to CIII 168 were displaced from each other by a translation of $\sim 3 \AA$ and a rotation of $\sim 37$ degrees (Fig. S3D, E;

169 SC-1A in red, and SC-1B in blue). Other subclasses identified in 3D classifications showed CIV in

170 various orientations between those seen in $\mathrm{SC}-1 \mathrm{~A}$ and $\mathrm{SC}-2 \mathrm{~B}$ maps. This $\mathrm{CIII}_{2} \mathrm{CIV}$ interface 171 flexibility is attributed to the limited interaction between the CcoP (N-ter TMH) of CIV and the cyt $172 b$ (TMH7) of $\mathrm{CIII}_{2}$ (Fig. 2, top view). In the interface regions of SC-1A and SC-1B maps, additional 173 weaker features that are not readily attributable to $\mathrm{CIII}_{2}$ and $\mathrm{CIV}$ structures were also observed. 174 However, no membrane-external feature that might correspond to cyt domain of $c_{\mathrm{y}}$, which is an 175 integral part of the cyt $c_{1}-\mathrm{CcoP}-c_{\mathrm{y}}$ subunit, could be discerned in these maps.

177 Structure of bipartite SC supplemented with cyt $c_{\mathbf{y}}$. The bipartite SC preparations (Fig. S1D, 178 fraction A-1) were supplemented with either purified full-length cyt $c_{\mathrm{y}}$, or its soluble variant lacking 179 the $\mathrm{TMH}$ (i.e., cyt S- $\left.c_{\mathrm{y}}\right)^{32}$, to yield the bipartite $\mathrm{SC}+c_{\mathrm{y}}$ and $\mathrm{SC}+\mathrm{S}-c_{\mathrm{y}}$ samples. Following $\mathrm{SEC}$, the 180 elution fractions showed that only the intact cyt $c_{\mathrm{y}}$, but not the cyt $\mathrm{S}-c_{\mathrm{y}}$, remained associated with the 181 SC (Fig. S4A). Thus, the cyt $c$ domain of $c_{\mathrm{y}}$ does not bind tightly to, and its TMH is required for 182 association with, the SC.

183 The cryo-EM analyses of the bipartite $\mathrm{SC}+c_{\mathrm{y}}$ yielded a map (SC-2A, EMD-22227) at 5.2A 184 resolution (Fig. S5A,B), with local resolutions ranging from 4.3-8.0Å (Fig. S6A,C). The homology 185 model of CIV and the refined model of $\mathrm{CIII}_{2}$ (PDB: 6XI0) were fitted as rigid bodies into SC-2A 
186 (Fig. 2A), with a correlation coefficient $\mathrm{CC}_{\mathrm{box}}$ of 0.74 (Table S3). Comparison of SC-2A (bipartite

$187 \mathrm{CIII}_{2} \mathrm{CIV}$ ) with SC-1A (tripartite $\mathrm{CIII}_{2} \mathrm{CIV}$ ) showed that these maps were highly similar, with RMSD

188 of $1.6 \AA$. Hence, they are collectively referred to as $\mathrm{CIII}_{2} \mathrm{CIV}$, irrespective of their bipartite or tripartite

189 origins.

190 The dimensions $(\sim 155 \times 60 \times 90 \AA, \mathrm{LxWxH})$ of the slightly curved $\mathrm{CIII}_{2} \mathrm{CIV}$ structure were

191 consistent with a $\mathrm{CIII}_{2}$ dimer associated with one CIV. On SC-2A map at $5.2 \AA$ resolution, some large

192 aromatic side chains could be readily discerned (Fig. 2B). Of the TMHs seen, 34 were accounted for

193 by two FeS proteins, two cyts $b$ and two cyts $c_{1}$ (2, 16 and 2 TMHs per dimer, respectively), and

194 single $\mathrm{CcoN}, \mathrm{CcoO}$ and $\mathrm{CcoP}$ (12,1 and $1 \mathrm{TMHs}$, respectively) (Fig. 2C). The features corresponding

195 to the heme cofactors of $\mathrm{CIII}_{2} \mathrm{CIV}$ were attributed to hemes $b_{\mathrm{H}}$ and $b_{\mathrm{L}}$ of cyt $b$, heme $c_{1}$ of cyt $c_{1}$, and

196 to hemes $b$ and $b_{3}$ of $\mathrm{CcoN}$, heme $c_{\mathrm{o}}$ of $\mathrm{CcoO}$ and hemes $c_{\mathrm{p} 1}$ and $c_{\mathrm{p} 2}$ of CcoP proteins. Like the tripartite

197 maps, the $[2 \mathrm{Fe}-2 \mathrm{~S}]$ clusters of $\mathrm{CIII}_{2}$ had lower resolution and were located closer to heme $b_{\mathrm{L}}$ (b

198 position). An additional TMH was observed at the distal end of CIV (Fig. 2A, rotated 180 degrees in

199 Fig. 2D) close to CcoN TMH3 and TMH4 (Fig. 2C). Due to its location, this TMH (depicted in Fig.

2002 as an ab initio model of CcoN Arg25-Leu48 residues) was tentatively attributed to the extra N-ter

201 TMH (i.e., TMH0) of CcoN, specific to R. capsulatus.

202 The interface of $\mathrm{CIII}_{2} \mathrm{CIV}$ is delimited by CcoN TMH8 and TMH9, CcoP TMH, cyt $b$ TMH5 and

203 TMH7, and cyt $c_{1}$ TMH of monomer A, with the closest interaction being between CcoP TMH and

204 cyt $b$ TMH7 (Fig. 2A,C). Two interacting TMHs of unknown identities (Fig. 2D, in red and blue),

205 and an inter-complex connection are seen at the interface. This connection is at the $n$ face of the

206 membrane, near the TMHs of cyt $c_{1}$ and CcoP (Fig. 2E, Lys $257_{c 1}$ and Thr $13_{\text {CcoP }}$ ), and tentatively

207 attributed to their covalent junction, linking $\mathrm{CIII}_{2}$ to $\mathrm{CIV}$. 
210 based approach predicting the residue-residue contacts in protein-protein interactions, RaptorX-

211 ComplexContact ${ }^{33}$, was used to identify the TMHs at the $\mathrm{CIII}_{2} \mathrm{CIV}$ interface. The single TMH

212 containing CIV-related proteins (i.e., $\mathrm{CcoQ}$ subunit, $\mathrm{CcoS}$ and $\mathrm{CcoH}$ assembly factors ${ }^{29}$ and cyt $c_{\mathrm{y}}{ }^{8}$ )

213 were analyzed against the subunits of $\mathrm{CIII}_{2}$ and CIV. Significant predictions of interacting residue

214 pairs (confidence value $>0.5$ ) were found only between CcoN (primarily TMH9) and the putative N-

215 term TMH of $\mathrm{CcoH}$ (Fig. S7A,B). An ab initio model of CcoH TMH was docked via PatchDock ${ }^{34}$

216 onto CIV using the predicted residue-residue contacts as distance restraints (15 ̊̊ threshold) (SI,

217 Methods). The top scoring models converged to a single cluster at the location of the unknown TMH,

218 next to CcoN TMH9 (Fig. S7C). Examination of the interactions between CcoH TMH and CcoN

219 TMH9 showed multiple co-evolutionarily conserved residues in close contacts (Fig. S7B,D). Earlier

220 studies had indicated that $\mathrm{CcoH}$ can be cross-linked by disuccinimidyl suberate (spacer length $\sim 11 \AA$ )

221 to $\mathrm{CcoP}$ and $\mathrm{CcoN}^{35}$. Thus, the $\mathrm{TMH}$ close to $\mathrm{CcoN}$ TMH9 (Fig. 2E, blue TMH) was tentatively 222 assigned to the assembly factor $\mathrm{CcoH}$.

223 An important difference between the maps SC-2A (bipartite $\mathrm{CIII}_{2} \mathrm{CIV}+c_{\mathrm{y}}$ ) and SC-1A (tripartite

$224 \mathrm{CIII}_{2} \mathrm{CIV}$ ) was seen at the interface. The unidentified TMHs were barely visible in SC-1 A, but highly

225 enhanced in SC-2A (Fig. 2E), indicating higher occupancy. The observation that only the native cyt

$226 c_{\mathrm{y}}$ binds to bipartite SC via its TMH suggested that the TMH (red in Fig. 2E) next to CcoH TMH

227 (blue in Fig. 2E) may correspond to the membrane-anchor of cyt $c_{\mathrm{y}}$. This explanation is supported by

228 fact that the bipartite $\mathrm{CIII}_{2} \mathrm{CIV}+c_{\mathrm{y}}$ was supplemented with full-length cyt $c_{\mathrm{y}}$ while the tripartite SC

229 contained only the fused cyt domain of $c_{y}$. Indeed, landmark densities corresponding to the helix-

230 breaking Gly11 and the correctly spaced bulky sides chains of Phe15 and Tyr21 of cyt $c_{\mathrm{y}} \mathrm{TMH}\left(\mathrm{NH}_{2^{-}}\right.$ 
We noted that some $\mathrm{CIII}_{2} \mathrm{CIV}+c_{\mathrm{y}}$ subclasses exhibited a weak feature on the $p$ side of the

233 membrane that may reflect the cyt domain of $c_{\mathrm{y}}$ (Fig. S5G, SC-2B). However, this feature could not

234 be refined to high resolution, consistent with the weak binding of cyt domain of $c_{\mathrm{y}}$ to $\mathrm{CIII}_{2} \mathrm{CIV}$.

235 Moreover, the predominant conformation of CIV in the bipartite $\mathrm{CIII}_{2} \mathrm{CIV}+c_{\mathrm{y}}($ Fig. S5A,B, SC-2A)

236 shifted towards that seen in map SC-1 A of tripartite SC (Fig. S2A), with no major class corresponding

237 to SC-1B (Fig. S2B). This suggested that local interactions between the CcoH and cyt $c_{\mathrm{y}}$ TMHs and

238 CIV decreased the interface flexibility of $\mathrm{CIII}_{2} \mathrm{CIV}$ (Fig. 2E).

240 Cryo-EM structures of $\boldsymbol{R}$. capsulatus native $\mathbf{C I I I}_{2}$. The bipartite $\mathrm{SC}+c_{\mathrm{y}}$ samples contained large 241 amounts of smaller particles ( 110Å length) (Fig. S2, Box 2) that were the size of CIII ${ }_{2}$ (Fig. S5C,D). 242 Analyses of these particles using $\mathrm{C} 2$ symmetry led to the map $\mathrm{CIII}_{2}$ at $3.3 \AA$ resolution for native $\mathrm{CIII}_{2}$ 243 (Fig. S5E), with local resolutions ranging from 3.0 to $4.0 \AA ̊$ (Fig. S6B,D) (Table S3). The FeS-ED

244 parts showed lower occupancy and resolution compared to the rest of the map, indicating 245 conformational heterogeneity. When similar analyses were carried out without imposing C2 246 symmetry, three distinct maps were obtained for $\mathrm{CIII}_{2}\left(\mathrm{CIII}_{2} \mathrm{c}-\mathrm{c}, \mathrm{CIII}_{2} \mathrm{~b}-\mathrm{c}\right.$ and $\mathrm{CIII}_{2} \mathrm{~b}-\mathrm{b}$ at 3.8, 4.2 247 and 3.5 ̊ resolutions, respectively) (Fig. S5F). These maps were superimposable with respect to cyt $248 b$ and cyt $c_{1}$ subunits, except for the FeS-ED portions. The $\mathrm{CIII}_{2}$ structures depicted by the $\mathrm{CIII}_{2} \mathrm{~b}-\mathrm{b}$ 249 (Fig. 3A-C) and $\mathrm{CIII}_{2} \mathrm{c}-\mathrm{c}$ (Fig. 3D) maps exhibited overall C2 symmetry, but in the former the FeS250 EDs were located in b, whereas in the latter in c position ${ }^{3}$. Notably, the third structure $\left(\mathrm{CIII}_{2} \mathrm{~b}-\mathrm{c}\right)$ was 251 asymmetric, with the FeS-ED of one monomer being in c, and the other one in b positions (Fig. 3E). 252 Such asymmetric structures of native $\mathrm{CIII}_{2}$ have not been observed frequently, although they are 253 proposed to occur during $\mathrm{QH}_{2}$ oxidation ${ }^{36-38}$. 
255 Interactions of cyt $\boldsymbol{c}_{\mathbf{2}}$ and cyt $\boldsymbol{c}_{\mathbf{y}}$ with $\mathrm{CIII}_{\mathbf{2}} \mathbf{C I V}$. The interaction interfaces between $\mathrm{CIII}_{2} \mathrm{CIV}$ and

256 its physiological electron carriers were analyzed by cross-linking mass spectrometry (XL-MS) ${ }^{39,40}$.

257 The co-crystal structure (PDB: $3 \mathrm{CX} 5$ ) of yeast $\mathrm{CIII}_{2}$ with its electron carrier iso- 1 cyt $c^{41}$ was used

258 as a template (homology between yeast cyt $c_{1}$ and $R$. capsulatus cyt $c_{1}: 31 \%$ identity and $58 \%$

259 similarity; iso-cyt $c$ and cyt $c_{2}: 25 \%$ identity and $56 \%$ similarity) to model the binding of cyt $c_{2}$ on

260 bacterial $\mathrm{CIII}_{2}$. The $R$. capsulatus cyt $c_{1}$ (PDB: 6XI0) and cyt $c_{2}$ (PDB: 1C2N) structures were

261 superimposed with their yeast counterparts on the co-crystal structure, and a model with a single cyt

$262 c_{2}$ docked to a monomer of $\mathrm{CIII}_{2}$ was obtained (Fig. S4C). To experimentally verify this model, the

263 protein cross-linker 4-(4,6-dimethoxy-1,3,5-triazin-2-yl)-4-methyl-morpholinium chloride

264 (DMTMM) was used (SI, Methods). The intra-subunit cross-links (XLs) within CIII ${ }_{2}$ CIV provided

265 controls, and high-confidence XLs were identified using both FindXL ${ }^{42}$ and MeroX ${ }^{43}$ search engines

266 (Table S4). The three XLs between cyt $c_{1}$ and cyt $c_{2}$ provided distance restraints ( 30Å for DMTMM)

267 for docking cyt $c_{2}$ to $\mathrm{CIII}_{2}$ (Table S4) (Fig. S4D), and the docking models clustered at a single region

268 per monomer of $\mathrm{CIII}_{2}$ (Fig. 4A, right). This region overlapped with the binding site of cyt $c_{2}$ defined

269 by the model obtained by alignment to the yeast co-crystal structure (Fig. S4E). The distance from

270 cyt $c_{2}$ heme-Fe to cyt $c_{1}$ heme-Fe was $\sim 16.8 \AA$ for the co-crystal derived model, while comparable

271 distances were between $\sim 13.8-20.4 \AA$ with the docking models. Thus, docking via Patchdock

272 integrating XL-MS mediated distance restraints defined reliably, but with limited accuracy, the

273 interaction region of cyt $c_{2}$ on $\mathrm{CIII}_{2}$.

274 No information about the binding of cyt $c_{2}$ and $c b b_{3}$-type CIV was available. This binding was

275 first shown biochemically (Fig. S4B), and then subjected to the XL-MS with DMTMM approach.

276 The XLs between the proteins ( 1 between cyt $c_{2}$ and CcoP, and 8 between cyt $c_{2}$ and CcoO) provided

277 distance restraints for docking cyt $c_{2}$ to CIV (Table S4). The docking models clustered in a single

278 region of CIV (Fig. $4 \mathrm{~A}$, left), closer to heme $c_{\mathrm{p} 2}\left(c_{2}\right.$ heme-Fe to $c_{\mathrm{p} 2}$ heme-Fe: $\sim 15.2$ to $\left.35.6 \AA\right)$ than 
279 heme $c_{\mathrm{p} 1}\left(c_{2}\right.$ heme-Fe to $c_{\mathrm{p} 1}$ heme-Fe: $\sim 23.0$ to $\left.42.0 \AA\right)$ of CcoP subunit (Fig. 5). The two cyt $c_{2}$ binding 280 regions on $\mathrm{CIII}_{2} \mathrm{CIV}$ are highly distant from each other (closest $c_{2}$ heme-Fe on $\mathrm{CIII}_{2}$ to that on $\mathrm{CIV}$ is $\sim 69 \AA ̊)$ (Fig. 5A).

282 Next, the binding interactions between cyt domain of $c_{\mathrm{y}}$ and $\mathrm{CIII}_{2} \mathrm{CIV}$ were sought using 283 DMTMM and disuccinimidyl dibutyric urea (DSBU) as cross-linkers. Similar to DMTMM, DSBU 284 yielded multiple intra-subunit XLs within the subunits of $\mathrm{CIII}_{2} \mathrm{CIV}$ as experimental controls (Table 285 S4). Six XLs (five cyt $c_{\mathrm{y}}$ to cyt $c_{1}$ and one cyt $c_{\mathrm{y}}$ to FeS protein) were found with DMTMM, four XLs 286 (only cyt $c_{\mathrm{y}}$ to FeS protein) with DSBU (Table S4), and none were observed between cyt $c_{\mathrm{y}}$ and CIV, 287 suggesting that cyt domain of $c_{\mathrm{y}}$ is closer to $\mathrm{CIII}_{2}$ in $\mathrm{CIII}_{2} \mathrm{CIV}$. In this case, PatchDock generated two 288 binding clusters for cyt domain of $c_{\mathrm{y}}$ on each $\mathrm{CIII}_{2}$ monomer of SC. One of the clusters was on cyt $c_{1}$ 289 and overlapped with cyt $c_{2}$ cluster (Fig. 4B), whereas the other one was between cyt $c_{1}$ and the FeS-

290 ED near $\mathrm{CIII}_{2}$ inter-monomer space (Fig. S4F-H). The two clusters 1 and 2 are best visible in a top 291 view (Fig. S4H), and the distances between cyt $c_{\mathrm{y}}$ heme-Fe and cyt $c_{1}$ heme-Fe of $\mathrm{CIII}_{2}$ monomer A 292 are between 13.8 to $47.1 \AA$ (Fig. 5). To further support these binding locations, we searched in our 293 cryo-EM datasets 3D classes that contained extra features corresponding to cyt domain of $c_{y}$. A minor 294 class ( 18,000 particles) (Fig. S5G) showing an extra feature attributable to cyt domain of $c_{\mathrm{y}}$ between 295 CIV and CIII was found (Fig. S4F,G).

296 Similar dockings of cyt domain of $c_{\mathrm{y}}$ were repeated using the different conformers of native $\mathrm{CIII}_{2}$ 297 (Fig. 3C-E, CIII 2 b-b, c-c and b-c). When the FeS-EDs are in c position ( $\left.\mathrm{CIII}_{2} \mathrm{c}-\mathrm{c}\right)$, the docking 298 models gathered as a single cluster on cyt $c_{1}$, slightly displaced towards the FeS-ED of the same 299 monomer (Fig. S8A-C). However, when the FeS-EDs are in b position $\left(\mathrm{CIII}_{2}\right.$ b-b), such models were 300 more spread out (Fig. S8D-F). The third model with one FeS-ED in c and the other in b positions 301 yielded the expected clustering pattern depending on the local FeS-ED conformation. In $\mathrm{CIII}_{2} \mathrm{CIV}$ 302 both FeS-EDs being in the b position, we assumed that cyt domain of $c_{\mathrm{y}}$ docks similarly to that seen 
303 with $\mathrm{CIII}_{2} \mathrm{~b}-\mathrm{b}$, and that the spreading of docking is due to the variable conformations of the FeS-EDs

304 in SC (Fig. S8). In addition, since heme $c_{1}$ and not the FeS protein is the electron exit site of CIII

$305{ }^{44,45}$, we imply that the cluster on cyt $c_{1}$ is the productive binding region of cyt domain of $c_{\mathrm{y}}$.

306 Examination of all pertinent distances between the cofactors of $\mathrm{CIII}_{2} \mathrm{CIV}$ (Fig. 5A) indicates that

307 the binding region of cyt domain of $c_{\mathrm{y}}$ near heme $c_{1}$ is far from CIV. The large distance $(\sim 50.8 \AA)$

308 separating cyt $c_{1}$ heme-Fe of $\mathrm{CIII}_{2}$ monomer A from $\mathrm{CcoP} c_{\mathrm{p} 1}$ heme-Fe (the closest compared with

309 heme $c_{\mathrm{p} 2}$ ) makes it impossible to define a position for cyt $c_{y}$ close enough to heme $c_{1}$ reducing it, and

310 heme $c_{\mathrm{p} 1}$ oxidizing it, while transferring electrons from $\mathrm{CIII}_{2}$ to $\mathrm{CIV}$. The distance constraint, the

311 inability to resolve the cyt domain of cyt $c_{\mathrm{y}}$ by cryo-EM, and the frequent $\mathrm{XLs}$ to $\mathrm{CIII}_{2}$ led us to

312 conclude that cyt $c_{\mathrm{y}}$ must oscillate within $\mathrm{CIII}_{2} \mathrm{CIV}$ during $\mathrm{QH}_{2}: \mathrm{O}_{2}$ oxidoreduction (Fig. 6). 


\section{Discussion}

314 Prior to this work, no structural information was available on any $c b b_{3}$-type CIV containing SC, or

315 its interactions with its physiological electron carriers. Here, we describe the first cryo-EM structures

316 of $\mathrm{CIII}_{2} \mathrm{CIV}$, a $b c_{1}-c b b_{3}$ type respiratory SC from the Gram-negative, facultative phototroph $R$.

317 capsulatus. We define the likely binding regions of cyt $c_{2}$ and cyt $c_{\mathrm{y}}$ to $\mathrm{CIII}_{2} \mathrm{CIV}$, and also report three

318 structural conformers of native $\mathrm{CIII}_{2}$ at high resolution. Although $3 \mathrm{D}$ structures of bacterial $b c_{1}$-type

$319 \mathrm{CIII}_{2}$ are available, native $\mathrm{CIII}_{2}$ heterodimers have not been reported. Similarly, only one structure

320 was available for $c b b_{3}$-type $\mathrm{CIV}{ }^{46}$. Members of this subfamily of heme-Cu: $\mathrm{O}_{2}$ reductases are

321 widespread among bacteria and essential for microaerobic processes, including anaerobic

322 photosynthesis and bacterial infection ${ }^{4}$. Unlike the obligate $\mathrm{CIII}_{2} \mathrm{CIV}_{2} \mathrm{SC}_{\text {of }}$ Actinobacteria, which

323 is rigid ${ }^{24,25}$, the facultative $\mathrm{CIII}_{2} \mathrm{CIV}$ of $R$. capsulatus is naturally flexible, limiting its structural

324 resolution. In such species, the dual function of $\mathrm{CIII}_{2}$, interacting with the photochemical reaction

325 center and CIV, may require this natural flexibility to allow swift metabolic adaptations.

326 Isolation of $\mathrm{CIII}_{2} \mathrm{CIV}$ was only possible using a genetically modified strain carrying a translational

327 fusion between $\mathrm{CIII}_{2}$ and $\mathrm{CIV}$ (SI, Methods). The fusion approach yielded compositionally

328 heterogeneous preparations, containing mixtures of $\mathrm{CIII}_{2} \mathrm{CIV}_{2}, \mathrm{CIII}_{2} \mathrm{CIV}$ and $\mathrm{CIII}_{2}$ particles, possibly

329 due to subunit sub-stoichiometry, incomplete assembly, or high susceptibility to degradation.

330 Insertion of different spacers at the cyt $c_{1}$-CcoP fusion junction, overexpression of the subunits and

331 related assembly components could not overcome this heterogeneity (SI, Methods). Consequently,

332 structural studies required extensive data collections, limited structural resolution, but allowed 333 analyses of fragmented particles.

334 Structures of $\mathbf{C I I I}_{2} \mathbf{C I V}$. The structures of the tripartite $\mathrm{CIII}_{2} \mathrm{CIV}$ or bipartite $\mathrm{CIII}_{2} \mathrm{CIV}+c_{\mathrm{y}}$ were

335 highly similar at sub-nanometer resolution ( 5.2 to $7.2 \AA)$. Their flexibility was due to limited protein-

336 protein interactions at their interface, where the TMHs of cyt $c_{\mathrm{y}}$ and $\mathrm{CcoH}$ are located (Fig. 2). 
337 Previously, neither the exact location nor the mobility of cyt $c_{\mathrm{y}}$ between $\mathrm{CIII}_{2}$ and CIV were known.

338 The SC structure shows that the N-terminal TMH of cyt $c_{\mathrm{y}}$ is locked at the interface, allowing mobility

339 of its cyt domain within $\mathrm{CIII}_{2} \mathrm{CIV}$ (Fig. 6). Although the linker region of cyt $c_{\mathrm{y}}$ remains unresolved,

340 it is long enough to reach both $\mathrm{CIII}_{2}$ and CIV. Earlier studies with $R$. capsulatus cyt $c_{\mathrm{y}}$ had shown that

341 a full-length linker (69 residues) is needed for rapid $(<\sim 50 \mu \mathrm{sec})$ electron transfer from $\mathrm{CIII}_{2}$ to the

342 photosynthetic reaction center ${ }^{47}$ whereas a shorter linker ( 45 -residues) is fully proficient for

343 respiratory electron transfer to $\mathrm{CIV}^{48}$.

344 Structures of bacterial native $\mathbf{C I I I}_{2}$. In native $\mathrm{CIII}_{2}$ conformers, different positions of the [2Fe-2S]

345 cluster bearing FeS-EDs were observed. Crystallographic structures have often depicted bacterial

$346 \mathrm{CIII}_{2}$ as symmetrical homodimers ${ }^{3,49,50}$. These structures were obtained in the presence of inhibitors

347 constraining FeS-EDs near heme $b_{\mathrm{L}}$ or used mutants stabilizing it on cyt $b$ surface. Alternatively, they

348 contained crystal contacts restricting the FeS-ED movement ${ }^{51}$. To our knowledge, no native

349 heterodimeric $\mathrm{CIII}_{2}$ structure of bacterial origin with different conformations of its FeS-EDs has been

350 reported. Only recent cryo-EM structures of mitochondrial SCs have shown different maps for $\mathrm{CIII}_{2}$

351 FeS-EDs ${ }^{17,52}$. Thus, native $\mathrm{CIII}_{2}$ is not always a symmetric homodimer, and the FeS-ED of each

352 monomer is free to move independently from the other. The Q-cycle models describe the mechanism

353 of $\mathrm{CIII}_{2}$ catalysis by two turnovers of a given monomer, with the different positions of the FeS-ED

354 protein attributed to different catalytic steps ${ }^{3,44,45}$. Emerging asymmetric structures of bacterial and

355 mitochondrial native $\mathrm{CIII}_{2}$ obtained by cryo-EM in the absence of inhibitors or mutations, combined

356 with the inter-monomer electron transfer between the hemes $b_{\mathrm{L}}$ of the monomers ${ }^{53,54}$, provide a

357 glimpse into plausible "heterodimeric Q cycle" mechanism(s) ${ }^{37,38}$. Accordingly, the FeS-EDs of CIII 2

358 monomers might cycle between homo- and hetero-dimeric conformations during the Q-cycle.

359 Electronic communication between CIII $_{2}$ CIV partners. This work defined hitherto unknown

360 structural interactions between the cyt $c_{2}$ or cyt $c_{\mathrm{y}}$ and $\mathrm{CIII}_{2} \mathrm{CIV}$ (Fig. 6). The $\mathrm{CIII}_{2} \mathrm{CIV}$ structure 
361 indicates that the distances separating heme $c_{1}$ of $\mathrm{CIII}_{2}$ monomer $\mathrm{A}$ and hemes $c_{\mathrm{p} 1}$ or $c_{\mathrm{p} 2}$ of CIV are 362 too large (Fig.5) for direct microsecond scale electronic communication ${ }^{55}$ to sustain the turnover rate 363 of $\mathrm{CIII}_{2} \mathrm{CIV}$. Even when $\mathrm{CIII}_{2}$ and CIV form a SC, an electron carrier is still required for $\mathrm{QH}_{2}: \mathrm{O}_{2}$ 364 oxidoreduction. The binding location of cyt $c_{2}$ on CIV defined here, the redox midpoint potentials $365\left(\mathrm{E}_{\mathrm{m}}\right)$ of the cofactors, and the distances separating them (Fig. 5A) suggest that cyt $c_{2}$ would confer 366 electrons to the closer heme $c_{\mathrm{p} 2}$, rather than the more distant heme $c_{\mathrm{p} 1}$, of CcoP. This will then initiate 367 canonical electron transfer via heme $c_{\mathrm{p} 1}$, heme $c_{\mathrm{o}}$ and heme $b$ to heme $b_{3}-\mathrm{Cu}_{\mathrm{B}}$ site for $\mathrm{O}_{2}$ reduction ${ }^{56,57}$ 368 (Fig. 6). For purified R. capsulatus proteins, the $\mathrm{E}_{\mathrm{m}}$ values of cyt $c_{2}$, cyt $c_{1}{ }^{30}$ and heme $c_{\mathrm{o}}$ of CcoO are 369 known ${ }^{31}$. The $\mathrm{E}_{\mathrm{m}}$ values of $R$. capsulatus $\mathrm{CcoP}$ hemes $c_{\mathrm{p} 1}$ and $c_{\mathrm{p} 2}$ are unknown, but based on similar $370 \quad \mathrm{E}_{\mathrm{m}}$ values of heme $c_{\mathrm{o}}$ for $B$. japonicum and $R$. capsulatus, they are expected to be similar ${ }^{58}$.

371 The likely interaction region of cyt $c_{\mathrm{y}}$ on cyt $c_{1}$ of $\mathrm{CIII}_{2}$, which is close to that of cyt $c_{2}$ is now 372 known, but that on CIV remains less well defined. The distance separating the redox centers is a major 373 factor that controls the rate of electron transfer ${ }^{55}$. The binding region of cyt domain of $c_{\mathrm{y}}$ on $\mathrm{CIII}_{2}$ 374 suggests that reduced cyt $c_{\mathrm{y}}$, upon its movement to CIV, might preferentially convey electrons to the 375 closer heme $c_{\mathrm{p} 1}$ than heme $c_{\mathrm{p} 2}$ of $\mathrm{CcoP}$ (Fig. 6). If so, under physiological conditions, heme $c_{\mathrm{p} 1}$ would 376 be the primary receiver of electrons derived from $\mathrm{QH}_{2}$ oxidation by $\mathrm{CIII}_{2}$, forming a fully membrane377 confined electronic wiring within $\mathrm{CIII}_{2} \mathrm{CIV}$. In contrast, cyt $c_{2}$ carries electrons from heme $c_{1}$ to heme $378 c_{\mathrm{p} 2}$ via free diffusion. This membrane-external pathway might accommodate electrons not only from $379 \mathrm{QH}_{2}$ but also from other donors distinct from $\mathrm{CIII}_{2}$. As such, reduction of cyt $c_{2}$ during methylamine 380 oxidation ${ }^{59}$, or degradation of sulfur containing amino acids, converting toxic sulfite $\left(\mathrm{SO}_{3}{ }^{2-}\right)$ to sulfate $381\left(\mathrm{SO}_{4}{ }^{-2}\right)$ by sulfate oxidase ${ }^{60}$ might provide electrons to $\mathrm{CIV}$, contributing to cellular energy 382 production.

383 In summary, the architecture of $\mathrm{CIII}_{2} \mathrm{CIV}$ along with its dynamics and interactions with its 384 physiological redox partners established salient structural features of two distinct respiratory electron 
385 transport pathways (membrane-confined and membrane-external) that operate between $\mathrm{CIII}_{2}$ and CIV 386 in Gram-negative bacteria for the first time. 


\section{Acknowledgments}

388 This work was supported partly by the NIH grants, GM 38237 to FD, GM123233 to KM, GM110174 389 and AI118891 to BAG, T32-GM008275 to TV, T32-GM071339 to HJK, and partly by the Division

390 of Chemical Sciences, Geosciences and Biosciences, Office of Basic Energy Sciences of Department

391 of Energy grant DE-FG02-91ER20052 to FD, by ISF 1466/18, BSF 2016070, and Ministry of Science

392 and technology 80802 grants to DS. YO was supported by the grant GRK2202-23577276/RTG from

393 DFG, Germany. Data analysis was partly supported by the NIH grant S10OD023592.

394 We thank Drs. S. Saif Hasan, Brian G. Pierce and Christian Presley at the Institute for Bioscience

395 and Biotechnology Research, University of Maryland, for insightful discussions and invaluable help

396 they provided during this study. SS and FD thank Vivian Kitainda for her assistance with protein

397 purification and $\mathrm{O}_{2}$ consumption measurements.

398 This research was supported in part by the NCI, National Cryo-EM Facility at the Frederick

399 National Laboratory for Cancer Research under contract HSSN261200800001E. The authors thank

400 Ulrich Baxa, Thomas Edwards and Adam Wier for their support and helpful discussions. Some cryo-

401 EM data were also obtained at the University of Massachusetts Cryo-EM Core Facility, and we thank

402 Drs. Chen Xu, KangKang Song and Kyounghwan Lee for their support. Cryo-EM sample screening 403 and optimization was performed at the Electron Microscopy Resource Laboratory at the Perelman 404 School of Medicine, University of Pennsylvania, and we thank Dr. Sudheer Molugu for his support. 


\section{Data deposition}

406 The following $R$. capsulatus structures and the corresponding cryo-EM maps are deposited to PDB 407 and EMDB: $\mathrm{CIII}_{2}$ (PDB: 6XI0; EMD-22189); $\mathrm{CIII}_{2}$ c-c (PDB: 6XKT; EMD-22224); $\mathrm{CIII}_{2}$ b-c (PDB:

408 6XKU; EMD-22225); CIII 2 b-b (PDB: 6XKV; EMD-22226); SC-2A (PDB: 6XKW; EMD-22227);

409 SC-1A (PDB: 6XKX; EMD-22228) and SC-1B (PDB: 6XKZ; EMD-22230). The raw XL-MS data

410 deposited to PRIDE repository (http://www.ebi.ac.uk/pride/archive/) with the dataset identifier $411 \quad$ PXD020038.

\section{Authors Contributions}

414 All authors have given approval to the final version of the manuscript. SS, TvE, YO, HJK, MB, NS, 415 BAG, DSD, KM and FD all contributed to aspects of the experiments, analyzed data, wrote and edited 416 the manuscript. SS purified, characterized and prepared samples, SS and TvE processed data and built 417 models, YO performed genetic constructs, MB and DSD conducted computer modeling, HJK, NS 418 and BAG obtained MS data, and KM and FD managed the overall project and supervised the study. 419 


\section{Methods}

\section{$421 \quad$ Bacterial strains and growth conditions}

422 Bacterial strains, plasmids and primers used are listed in Table S1. LB medium supplemented as 423 appropriate with ampicillin, gentamicin, kanamycin or tetracycline at $100,12,50$ or $12.5 \mu \mathrm{g} / \mathrm{mL}$, 424 respectively, was used for growing E. coli strains at $37{ }^{\circ} \mathrm{C}$. $R$. capsulatus strains were grown 425 chemoheterotrophically under semi-aerobic/dark conditions at $35^{\circ} \mathrm{C}$ on enriched (MPYE) medium, 426 supplemented as needed with gentamicin, kanamycin, spectinomycin, or tetracycline at 3, 10, 10 or $4272.5 \mu \mathrm{g} / \mathrm{mL}$, respectively. Colonies were stained for $c b b_{3}$-type CIV activity by incubating plates with 428 a 1:1 (v/v) mixture of $35 \mathrm{mM}$ 1-naphtol and $30 \mathrm{mM} \mathrm{N}, \mathrm{N}$-dimethyl-1,4-phenylenediamine (NADI429 staining) ${ }^{61}$.

\section{$431 \quad$ Molecular genetic techniques}

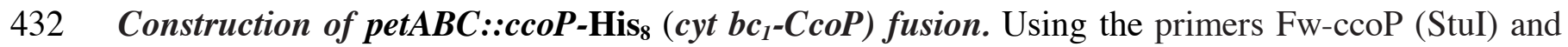

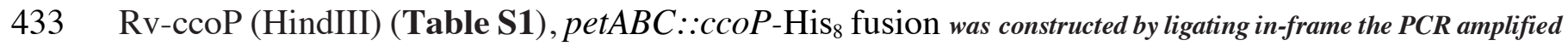
$4341.13 \mathrm{~kb}$ StuI-HindIII fragment containing $c c o P$ (with a C-terminal His $\mathrm{s}_{8}$ tag) to the 3' end of petABC, after 435 elimination of the stop codon of petC and start codon of ccoP on StuI-HindIII digested plasmid pMTSI, to yield 436 pYO60 (Table S1). The $3.85 \mathrm{~kb}$ BamHI fragment of pYO60 carrying petABC::ccoP fusion was 437 transferred to the plasmids pBSII and pRK415 using the same sites, yielding pYO63 and pYO76, 438 respectively. The linker-spaced versions of petABC::ccoP fusion (petABC::L2-ccoP, petABC::L3439 cсoP and petABC::L4-ccoP) were also constructed by exchanging the StuI-HindIII fragment of pYO63 440 carrying petABC-ccoP with the PCR amplified linker added versions, yielding pYO77, pYO78 and 441 pYO80, respectively. L2, L3 and L4 linkers were introduced via the primers F-L2, F-L3 and F-L4

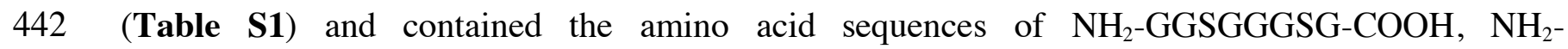


$444 \mathrm{XbaI}$ fragments carrying these linkers added versions were cloned to pRK415, yielding pYO81, 445 pYO82 and pYO83, respectively. All constructs were subsequently verified by DNA sequencing. As the protein yields and enzymatic activities of all fusion super-complexes (SCs) were similar, only pYO76, which has no linker (i.e., native-like fusion), 447 was used for subsequent work.

448 Construction of petABC::ccoP::cycY-Flag (cyt $\boldsymbol{b c}_{1}-$ CcoP-cyt $\boldsymbol{c}_{y}$-Flag) fusion. Using the primers

449 Fw-cp-cy (BalI) and Rv-cp-cy (BglII+HinIII), the C-terminally Flag tagged cycY lacking its $\mathrm{N}$ 450 terminal TMH anchor (i.e., amino acid residues 1-30) was PCR amplified. The 619 bp long PCR 451 fragment containing $c y c Y$-Flag without its anchor was digested with BalI-HinIII and exchanged with 452 its counterpart that encompasses the 3 ' end of $c c o P$ on pYO63 to yield the petABC::ccoP ::cycY-Flag 453 fusion carried by pYO91. The $4.5 \mathrm{~kb} \mathrm{KpnI}-\mathrm{XbaI}$ fragment of pYO91 containing this fusion was 454 cloned into pRK415 using the same sites to yield pYO92 (Table S1).

455 Chromosomal inactivation of $\mathrm{CIII}_{2}\left(\mathbf{c y t} \boldsymbol{b c}_{1}\right)$ and $\mathrm{CIV}\left(\boldsymbol{c b} \boldsymbol{b}_{3}\right.$-type Cox $)$ structural genes, and 456 construction of strains producing bipartite and tripartite fusion SCs. The $\triangle(p e t A B C: \because g m)$ 457 deletion-insertion allele carried by pYO34 ${ }^{32}$ was transferred using the gene transfer agent (GTA) into 458 the chromosome of $R$. capsulatus strain MG1 (ccoP::kan) to yield YO12, providing a mutant 459 background lacking both $\mathrm{CIII}_{2}$ and the $\mathrm{CcoP}$ subunit of CIV. A similar strain, M7G-CBC1 that lacks 460 both $\mathrm{CIII}_{2}$ and the $\mathrm{CcoP}$ subunit but overproduces the $\mathrm{CcoN}$ and $\mathrm{CcoO}$ subunits of $\mathrm{CIV}^{31}$, was also 461 used. The plasmids pYO76 and pYO92 encoding the bipartite and tripartite SCs, respectively, were 462 conjugated into YO12 and M7G-CBC1 using triparental mating to yield pYO76/YO12 and 463 pYO92/M7G-CBC1 strains as in ${ }^{61}$ for protein purification. 
466 Protein purification. $R$. capsulatus cells $(\sim 25-30 \mathrm{~g}$ from $8 \mathrm{~L}$ media) were resuspended in a final

467 volume of 35-40 mL of buffer A (50 mM Tris- $\mathrm{mCl}, \mathrm{pH} 8.0,100 \mathrm{mM} \mathrm{NaCl}, 20 \mathrm{mM}$ EDTA, $1 \mathrm{mM}$

468 aminocaproic acid and $1 \mathrm{mM}$ 4-(2-Aminoethyl) benzenesulfonyl fluoride hydrochloride (AEBSF)

469 supplemented with Pierce Protease Inhibitor (Thermo Scientific, 1 mini tablet per $30 \mathrm{~mL}$ ). Cells were

470 disrupted by three passages through a French Pressure Cell (SLM Aminco) at 13,000 psi in the

471 presence of 5-10 mg of DNase I (GoldBio), and cell debris removed by centrifugation at 27,000 x $\mathrm{g}$

472 for $30 \mathrm{~min}^{31}$. Chromatophore membranes were sedimented by centrifugation at 190,000 x $\mathrm{g}$ for $2 \mathrm{~h}$

473 and resuspended in buffer A. The protein concentration was determined using the Pierce Protein BCA

474 assay kit (Thermo Scientific), the suspension was supplemented with $10 \%$ glycerol and adjusted to a

475 protein concentration of $10 \mathrm{mg} / \mathrm{mL}$ with buffer A. Membrane proteins were solubilized with $2 \% \mathrm{n}-$

476 dodecyl-ß3-D-maltoside (DDM, Anatrace) for $15 \mathrm{~min}$ on ice, and non-solubilized materials

477 sedimented by centrifugation at $100,000 \mathrm{~g}$ for $15 \mathrm{~min}$. The supernatant was subjected to

478 chromatography, as appropriate.

479 The His-tagged bipartite SC was purified by anion exchange chromatography on Bio-Gel A-50

480 (Bio-Rad), followed by affinity chromatography on Ni-Sepharose High Performance (GE

481 Healthcare). Solubilized proteins were loaded onto a $40 \mathrm{~mL}$ Bio-Gel column equilibrated with Tris

482 buffer (50 mM Tris-HCl, $\mathrm{pH} 8.0,100 \mathrm{mM} \mathrm{NaCl}, 1 \%$ glycerol and $0.01 \%$ DDM) and washed with

483 three column volumes (CV) of the buffer. After another washing step with $3 \mathrm{CV}$ of Tris buffer

484 containing $150 \mathrm{mM} \mathrm{NaCl}$ to eliminate weakly bound proteins elution was carried out with a linear,

485 five $\mathrm{CV}$ gradient from $150-400 \mathrm{mM} \mathrm{NaCl}$ in Tris buffer. Fractions containing both cyt $c$ reductase 486 and cyt $c$ oxidase activities were combined and loaded onto a $7 \mathrm{~mL} \mathrm{Ni-Sepharose} \mathrm{column} \mathrm{equilibrated}$ 487 with the binding buffer $(50 \mathrm{mM}$ Tris- $\mathrm{HCl}, \mathrm{pH} 8.0,500 \mathrm{mM} \mathrm{NaCl}, 20 \mathrm{mM}$ imidazole, $1 \%$ glycerol and $4880.01 \%$ DDM). After washing with $30 \mathrm{~mL}$ of binding buffer, the column was eluted with a linear 100 $489 \mathrm{~mL}$ gradient from 20-260 mM imidazole. 
491 gel (Bimake). The supernatant containing solubilized proteins was loaded onto a $1 \mathrm{~mL}$ column 492 equilibrated with TBS buffer (50 mM Tris-HCl, pH 7.4, $150 \mathrm{mM} \mathrm{NaCl,} 0.01 \%$ DDM and $1 \mathrm{mM}$ 493 AEBSF), washed with $10 \mathrm{ml}$ TBS and eluted with $5 \mathrm{~mL}$ of Flag (DYKDDDDK) peptide $(100 \mu \mathrm{g} / \mathrm{mL})$ 494 (Sigma) in TBS buffer.

495 Eluted proteins were concentrated (Amicon Ultra-15, $30 \mathrm{kDa}$ MWCO) (Millipore) to a final 496 volume of 150-200 $\mu \mathrm{L}$ and loaded onto a Superose 6 Increase 10/300 GL (GE Healthcare) size 497 exclusion column equilibrated with TBS buffer, and eluted with the same buffer at a flow rate of 0.4 $498 \mathrm{~mL} / \mathrm{min}$. Peak fractions were combined and concentrated, and PD MiniTrap G-25 columns (GE 499 Healthcare) were used for buffer exchanges, as needed.

$500 \quad$ Protein analyses. Native PAGE was performed using 4-13\% gels ${ }^{62}$, and SDS-PAGE used $12.5 \%$ 501 or $18 \%$ gels ${ }^{63}$. Gels were stained with Coomassie Brilliant Blue (Bio-Rad) or colloidal silver ${ }^{64}$. 502 Immunoblot analyses were performed using polyclonal antibodies specific of $R$. capsulatus cyt $b^{28}$ 503 and monoclonal anti-rabbit-IgG-alkaline phosphatase (Sigma) used at 1:10.000 dilution. CIV in-gel 504 activity was revealed by incubating native gels with $0.5 \mathrm{mg} / \mathrm{ml} \mathrm{3,3}$ '-diaminobenzidine in $50 \mathrm{mM} \mathrm{Na}$ 505 phosphate, $\mathrm{pH} 7.2^{65}$. The $c$-type cyts were revealed by their heme peroxidase activity, using $3,3^{\prime}, 5,5^{\prime}-$ 506 tetramethylbenzidine (TMBZ) and hydrogen peroxide $\left(\mathrm{H}_{2} \mathrm{O}_{2}\right)^{66}$. Briefly, gels were washed with 0.25 507 M Na-acetate buffer ( $\mathrm{pH}$ 5) and incubated with $6.3 \mathrm{mM}$ TMBZ in 30\% methanol, $0.25 \mathrm{M} \mathrm{Na}$-acetate, $508 \mathrm{pH}$ 5.0. After $1 \mathrm{~h}$ incubation, $0.4 \% \mathrm{H}_{2} \mathrm{O}_{2}$ were added to reveal the peroxidase activity of $c$-type cyts. 509 Reduced minus oxidized spectra were recorded in $50 \mathrm{mM}$ MOPS, pH 7.0 using a Cary 60 UV510 Vis spectrophotometer (Agilent). Usually, $50 \mu \mathrm{g}$ of purified protein was first fully oxidized by adding 511 a grain of $\mathrm{K}_{3}\left[\mathrm{Fe}(\mathrm{CN})_{6}\right]$ and then gradually reduced with a few grains of sodium ascorbate or sodium 512 dithionite, as appropriate. Protein concentrations were determined with a NanoDrop 2000c (Thermo 513 Scientific) using the $\mathrm{A}_{280}$ method ${ }^{67}$. 
514 Enzyme activities. Cyt $c$ reductase (cyt $b c_{1}$ ) activity was determined as described ${ }^{68}$. Briefly, 10

$515 \mathrm{mM}$ stock solution of 2,3-dimethoxy-5-methyl-6-decyl-1,4-benzoquinone (DB) (Sigma) in DMSO

516 was reduced to $\mathrm{DBH}_{2}$ with a few grains of sodium borohydride and excess borohydride was quenched

517 by adding $\mathrm{HCl}$ to a final $\mathrm{pH}$ of 6.0 . For assays, $40 \mu \mathrm{M} \mathrm{DBH} \mathrm{DH}_{2}$ were added to $25 \mu \mathrm{M}$ horse heart cyt $c$

518 (Sigma) in $500 \mu \mathrm{L}$ assay buffer (40 mM sodium phosphate, $\mathrm{pH} 7.4,20 \mathrm{mM}$ sodium malonate, 0.5

$519 \mathrm{mM}$ EDTA, $0.5 \mathrm{mM} \mathrm{KCN}$ and $0.01 \%$ DDM) in a stirred cuvette, and the non-enzymatic rate of cyt $c$

520 reduction was recorded at $550 \mathrm{~nm}$ for $1 \mathrm{~min}$ (Cary $60 \mathrm{UV}$-Vis spectrophotometer, Agilent). The

521 reaction was initiated by adding $1-2 \mu \mathrm{g}$ of purified protein and cyt $c$ reduction was monitored. The

522 enzymatic activity was inhibited by adding $1 \mu \mathrm{M}$ stigmatellin (Fluka), a specific inhibitor of cyt $b c_{1^{-}}$

523 type $\mathrm{CIII}_{2}$.

524 The CIV (cyt $c b b_{3}$-Cox) activity was determined as described ${ }^{69}$. Reduced horse heart cyt $c$ 525 (Sigma) was prepared by incubating a 1-2 $\mathrm{mM}$ stock solution with $10 \mathrm{mM}$ sodium dithionite for 15 $526 \mathrm{~min}$ at room temperature, excess dithionite was removed by gel filtration using a PD-10 desalting 527 column (GE Healthcare), and the final concentration of reduced cyt $c$ was calculated based on its 528 absorption at $550 \mathrm{~nm}$ and an $\varepsilon=20 \mathrm{mM}^{-1} \mathrm{~cm}^{-1} .20 \mu \mathrm{M}$ reduced cyt $c$ in $500 \mu \mathrm{L}$ assay buffer $(10 \mathrm{mM}$

529 Tris- $\mathrm{HCl}, \mathrm{pH} 7.0,100 \mathrm{mM} \mathrm{KCl}$ ) were prepared in a stirred cuvette (Cary $60 \mathrm{UV}$-Vis

530 spectrophotometer), and the baseline was recorded for $1 \mathrm{~min}$. The reaction was started by adding 1-2

$531 \mu \mathrm{g}$ of purified protein, and cyt $c$ oxidation monitored at $550 \mathrm{~nm}$. The enzymatic activity was inhibited

532 by addition of $250 \mu \mathrm{M} \mathrm{KCN}$ as an inhibitor of $c b b_{3}$-Cox.

$533 \mathrm{DBH}_{2}$ dependent oxygen consumption was monitored using a mini Clark-type oxygen electrode 534 (Instech Laboratories, PA) at $25^{\circ} \mathrm{C}$. The assay was performed in the same buffer as that used for cyt $535 c$ reductase activity $(40 \mathrm{mM}$ sodium phosphate, $\mathrm{pH} 7.4,20 \mathrm{mM}$ sodium malonate, $0.5 \mathrm{mM}$ EDTA and $5360.01 \% \mathrm{DDM}$ ), but without $\mathrm{KCN}$. A baseline was recorded after adding $100 \mu \mathrm{M} \mathrm{DBH} \mathrm{D}_{2}$ to $1 \mathrm{~mL}$ of 537 assay buffer, and the reaction started by adding $30 \mu \mathrm{g}$ of purified protein. As needed, purified protein 
was pre-incubated with a 1:1 (w/w) mixture of E. coli polar lipids (Avanti), which improved the

539 activity. $20 \mu \mathrm{M}$ horse heart cyt $c$ (Sigma), $250 \mu \mathrm{M} \mathrm{KCN}$ or $1 \mu \mathrm{M}$ of stigmatellin were used as

540 specificity controls.

541 Identification of protein subunits by mass spectrometry. Protein bands from gels were excised,

542 and after reduction (dithiothreitol; Sigma) and alkylation (iodoacetamide; Bio-Rad) subjected to in-

543 gel trypsin digestion (Promega, Sequencing Grade Modified Trypsin) overnight at $37^{\circ} \mathrm{C}$. Peptides

544 eluted from the gel samples were dried, desalted using ZipTips (Millipore U-C18 P10, Millipore),

545 lyophilized and stored at $-80{ }^{\circ} \mathrm{C}$. They were resuspended in $10 \mu \mathrm{L} 5 \%$ acetonitrile/0.1\% formic acid

546 prior to MS, using either a LCQ Deca XP+ ion trap, or a Q-Exactive Quadrupole-Orbitrap mass

547 spectrometer (both from Thermo Fisher Scientific) ${ }^{70}$. The LCQ Deca XP+ mass spectrometer was

548 coupled to a Thermo-Dionex LC Packings Ultimate Nano HPLC system controlled by Thermo

549 Xcalibur version 2.0 software. Peptides were separated on a $15 \mathrm{~cm} \mathrm{C18}$ nanocolumn (Thermo-

550 Dionex, NAN-75-15-03-PM) using a 45-min linear gradient from $4 \%$ to $40 \%$ buffer B (100\%

551 acetonitrile, $0.1 \%$ formic acid), followed by a 7 -min gradient from $40 \%$ to $80 \%$ buffer B and 8 -min

552 wash with $80 \% \mathrm{~B}$ (constant flow rate $150 \mathrm{~nL} \cdot \mathrm{min}^{-1}$ ). MS/MS data were acquired in data-dependent

553 analysis mode with dynamic exclusion enabled (repeat count: 3, exclusion duration: 3 min). Full MS

554 survey scans (mass range 300-2000 m/z) were followed by MS/MS fragmentation (normalized

555 collision energy 35) of the top 3 most intense ions. The Q-Exactive Quadrupole-Orbitrap mass

556 spectrometer was coupled to an Easy-nLC ${ }^{\mathrm{Tм}} 1000$ nano HPLC (Thermo Fisher Scientific), and

557 samples were loaded in buffer A ( $0.1 \%$ formic acid $)$ onto a $20-\mathrm{cm}-$ long fused silica capillary column

558 (75 $\mu \mathrm{m}$ ID), packed with reversed-phase Repro-Sil Pur C18-AQ $3 \mu \mathrm{m}$ resin (Dr. Maisch GmbH,

559 Ammerbuch, Germany). Peptides were eluted using a 45-min linear gradient from $4 \%$ to $40 \%$ buffer

560 B (100\% acetonitrile, $0.1 \%$ formic acid), followed by a 7 -min gradient from $40 \%$ to $80 \%$ buffer B

561 and 8-min wash with $80 \% \mathrm{~B}$ (constant flow rate $300 \mathrm{~nL} \cdot \mathrm{min}^{-1}$ ). The Q-Exactive was operated in data- 
562 dependent acquisition mode with dynamic exclusion enabled (repeat count: 1, exclusion duration: 20

563 s). Full MS survey scans (mass range $300-1600 \mathrm{~m} / \mathrm{z}$ ) at high resolution $(70000$ at $200 \mathrm{~m} / \mathrm{z}$ ) were

564 followed by MS/MS fragmentation of the top 15 most intense ions with higher energy collisional 565 dissociation at a normalized collision energy of 22 (resolution 17500 at $200 \mathrm{~m} / \mathrm{z}$ ). Dual lock mass 566 calibration was enabled with 371.101233 and $445.120024 \mathrm{~m} / \mathrm{z}$ background ions.

567 MS spectra were searched against the $R$. capsulatus protein database (https://www.uniprot.org, 568 last modified 01/15/2020; Rhodobacter capsulatus (strain ATCC BAA-309 / NBRC 16581 /

569 SB1003)) using Proteome Discoverer 1.4 (Thermo Fisher Scientific) with Sequest-HT search engine.

570 Search parameters were set to full trypsin digestion, with maxima of three missed cleavages and three

571 modifications per peptide. Oxidation of methionine (+16 Da) and carbamidomethylation of cysteine

572 (+57 Da) were selected as dynamic modifications. Precursor and fragment ion tolerances were set to

$5732 \mathrm{Da}$ and $1 \mathrm{Da}$, respectively, for the LCQ DecaXP+, and $10 \mathrm{ppm}$ and 0.6 Da, respectively, for the Q-

574 Exactive data. False discovery rates by target-decoy search (FDR) were set to 0.01 and $\mathrm{X}_{\text {corr }}$ filter

575 based on charge (z) were: $>2$ for $z=2 ;>2.5$ for $z=3$; and $>2.6$ for $z=4{ }^{70}$. All identifications are

576 listed in Table S2.

577

578 Binding of cyt $c_{y}$, soluble variant of cyt $c_{y}\left(c y t S-c_{y}\right)$ and cyt $c_{2}$ to purified proteins

579 Cyt $c_{\mathbf{y}}$ and cyt S- $\boldsymbol{c}_{\mathbf{y}}$. Binding of purified cyt $c_{\mathrm{y}}{ }^{71}$, or a variant of it containing only the soluble cyt 580 domain (cyt S- $c_{y}$ ) (residues 99-199) to the bipartite SC was assayed by mixing a 5-10 fold molar 581 excess of purified cyt $c$ with $125 \mu \mathrm{g}$ of purified SC in a final volume of $150 \mu \mathrm{L}$ TBS buffer $(50 \mathrm{mM}$ 582 Tris- $\mathrm{HCl}, \mathrm{pH} 7.4,150 \mathrm{mM} \mathrm{NaCl}$ and $0.01 \% \mathrm{DDM}$ ). The mixture was separated by chromatography 583 using Superose 6 Increase size exclusion column, and elution fractions were analyzed by SDS-PAGE 584 followed by silver staining. Cyt S- $c_{\mathrm{y}}$ was purified from the $R$. capsulatus strain pYO135/FJ2-R4 585 (Table S1) grown under photosynthetic conditions to maximize its yield ${ }^{32}$. Cells were washed with 
$20 \mathrm{mM}$ Tris- $\mathrm{HCl}, \mathrm{pH} 8.0$ and resuspended 1:5 (w/v) in the same buffer supplemented with $50 \mathrm{mM}$

$587 \mathrm{NaCl}$. Polymyxin B sulfate $(1 \mathrm{mg} / \mathrm{mL})$ was added, and the cell suspension incubated for $75 \mathrm{~min}$ on 588 ice with gentle stirring. After centrifugation for $20 \mathrm{~min}$ at $10,000 \mathrm{~g}$ followed by $3 \mathrm{~h}$ at 150,000 $\mathrm{g}$, the 589 supernatant was collected and concentrated to a final volume of $2 \mathrm{ml}$ (Amicon Ultra-15 $3 \mathrm{kDa}$ 590 MWCO) (Millipore). Aliquots of $300 \mu \mathrm{L}$ were loaded onto Superose 6 Increase (GE Healthcare) 591 equilibrated in $30 \mathrm{mM}$ Tris- $\mathrm{HCl}, \mathrm{pH} 8.0$ and eluted in the same buffer. Fractions eluting after $20 \mathrm{~mL}$ 592 were combined and concentrated using Amicon Ultra-15 3 kDa MWCO filters (Millipore).

593 Cyt $c_{2}$. Binding of cyt $c_{2}$ to $c b b_{3}$-type CIV was determined by mixing 2-fold molar excess of 594 purified $R$. capsulatus cyt $c_{2}{ }^{72}$ with $300 \mu$ g of purified CIV ${ }^{31}$ under low-salt conditions (20 mM Tris$595 \mathrm{HCl}, \mathrm{pH} 7.4,1 \mathrm{mM} \mathrm{NaCl}$ and $0.01 \% \mathrm{DDM})$. The mixture (250 $\mu \mathrm{L}$ total volume) was loaded onto a

596 Superose 6 Increase (GE Healthcare) sizing column equilibrated with the same buffer to separate the 597 proteins. Elution fractions were concentrated (Amicon Ultra-15, 3 kDa MWCO) (Millipore) and a 598 volume containing 1 to $5 \mu \mathrm{g}$ of protein was analyzed by SDS-PAGE followed by silver staining.

\section{Protein cross-linking and mass spectrometry}

601 Protein cross-linking using chemical crosslinkers. $180 \mu \mathrm{g}$ of purified bipartite SC at a 602 concentration of $1 \mathrm{mg} / \mathrm{mL}$ in PBS buffer (50 mM Na-Phosphate, $\mathrm{pH} 7.4,150 \mathrm{mM} \mathrm{NaCl}$ and $0.01 \%$ 603 DDM) mixed with 5-fold molar excess $(90 \mu \mathrm{g})$ of purified cyt $c_{\mathrm{y}}{ }^{71}$ were supplemented with $6 \mathrm{mM}$ 604 disuccinimidyl dibutyric urea (DSBU) (Thermo Fisher Scientific), and incubated on ice for $2 \mathrm{~h}$. The 605 reaction was quenched by adding $50 \mathrm{mM}$ of ammonium bicarbonate, and the mixture analyzed by 606 mass spectrometry. $300 \mu \mathrm{g}$ of purified $c b b_{3}$-type CIV ${ }^{31}$ were mixed with a 2-fold molar excess of 607 purified cyt $c_{2}{ }^{72}$ at a final protein concentration of $200 \mu \mathrm{g} / \mathrm{mL}$ under low-salt conditions (20 mM Na608 phosphate, $\mathrm{pH} 7.4,1 \mathrm{mM} \mathrm{NaCl}$ and $0.01 \% \mathrm{DDM})$, and incubated with $20 \mathrm{mM}$ 4-(4,6-Dimethoxy609 1,3,5-triazin-2-yl)-4-methylmorpholinium chloride (DMTMM) (Sigma) for $1 \mathrm{~h}$ at room temperature. 
610 Similarly, $300 \mu \mathrm{g}$ of purified $b c_{1}$-type $\mathrm{CIII}_{2}$ were mixed with 2-fold molar excess of purified cyt $c_{2}$,

611 and treated with DMTMM as above. In both cases, the reaction was stopped by removing excess

612 DMTMM with a PD MiniTrap G-25 desalting column (GE Healthcare), and crosslinked proteins

613 were precipitated with $20 \%(\mathrm{w} / \mathrm{v})$ trichloroacetic acid (TCA, Sigma) at $4^{\circ} \mathrm{C}$ for $1 \mathrm{~h}$. Proteins were

614 pelleted by centrifugation at 21,000 $\mathrm{x}$ g for $15 \mathrm{~min}$ and washed with $10 \% \mathrm{TCA}$ in $0.1 \mathrm{M}$ Tris- $\mathrm{HCl}$ and

615 then with acetone (Fisher). The solvent was discarded, the pellet air-dried and then stored at $-80^{\circ} \mathrm{C}$

616 for analysis by mass spectrometry.

617 Mass spectrometry of crosslinked proteins. Crosslinked proteins were resuspended in an 618 appropriate volume of solution A $(2.5 \%$ TCA, $50 \mathrm{mM}$ SDS and $50 \mathrm{mM}$ triethylammonium 619 bicarbonate (TEAB) final concentrations) and reduced with $10 \mathrm{mM}$ DTT (US Biological) for $30 \mathrm{~min}$ 620 at $30^{\circ} \mathrm{C}$, followed by alkylation with $50 \mathrm{mM}$ iodoacetamide (Sigma Aldrich) for $30 \mathrm{~min}$ at $30^{\circ} \mathrm{C}$. The 621 proteins were processed using an S-Trap ${ }^{\mathrm{TM}}$ according to the protocol recommended by the supplier 622 (Protifi, C02-mini), and digested with trypsin (Thermo Fisher Scientific) in 1:10 (w/w) 623 enzyme/protein ratio for $1 \mathrm{~h}$ at $30^{\circ} \mathrm{C}$. Peptides eluted from this column were vacuum-dried and 624 resuspended with the peptide fractionation-elution buffer for LC-MS [(70\% (v/v) LC-MS grade water 625 (Thermo Fisher Scientific), 30\% (v/v) acetonitrile (ACN, Thermo Fisher Scientific) and $0.1 \%$ (v/v) 626 trifluoroacetic acid (TFA, Thermo Fisher Scientific)]. Peptides were first fractionated using AKTA 627 Pure 25 with Superdex 30 Increase 3.2/300 (GE Life Science) at a flow rate of $30 \mu \mathrm{L} \mathrm{min}^{-1}$ of the 628 elution buffer, and $100 \mu \mathrm{L}$ fractions were collected. Based on the elution profile, fractions containing 629 enriched crosslinked peptides of higher molecular masses, were vacuum-dried and resuspended with 630 LC-MS grade water containing $0.1 \%$ (v/v) TFA for mass spectrometry analysis. One half of each 631 fraction was analyzed by a Q-Exactive HF mass spectrometer (Thermo Fisher Scientific) coupled to 632 a Dionex Ultimate 3000 UHPLC system (Thermo Fischer Scientific) equipped with an in-house made $63315 \mathrm{~cm}$ long fused silica capillary column (75 $\mu \mathrm{m}$ ID), packed with reversed-phase Repro-Sil Pur C18- 
634 AQ 2.4 $\mu \mathrm{m}$ resin (Dr. Maisch GmbH, Ammerbuch, Germany). Elution was performed using a 635 gradient from 5\% to $45 \%$ B (90 min), followed by $90 \%$ B (5 min), and re-equilibration from $90 \%$ to $6365 \% \mathrm{~B}(5 \mathrm{~min})$ with a flow rate of $400 \mathrm{~nL} / \mathrm{min}$ (mobile phase A: water with $0.1 \%$ formic acid; mobile

637 phase B: $80 \%$ acetonitrile with $0.1 \%$ formic acid). Data were acquired in data-dependent MS/MS 638 mode. Full scan MS settings were: mass range 300-1800 m/z, resolution 120,000; MS1 AGC target 639 1E6; MS1 Maximum IT 200. MS/MS settings were: resolution 30,000; AGC target 2E5; MS2 640 Maximum IT $300 \mathrm{~ms}$; fragmentation was enforced by higher-energy collisional dissociation with 641 stepped collision energy of 25,27,30; loop count top 12; isolation window 1.5; fixed first mass 130; 642 MS2 Minimum AGC target 800; charge exclusion: unassigned, 1, 2, 3, 8 and >8; peptide match off; 643 exclude isotope on; dynamic exclusion $45 \mathrm{sec}^{39}$. Raw files were converted to mgf format with 644 TurboRawToMGF 2.0.8 ${ }^{73}$.

645 Crosslinked peptide searches. MeroX 2.0.0.5 ${ }^{40}$, FindXL ${ }^{42}$ and MassAI 19.07 646 (http://www.massai.dk) were used to identify and validate crosslinked peptides, and only those that 647 were consistently identified by two different search engines (MeroX and FindXL for DMTMM, and 648 MeroX and MassAI for DSBU) were used for method validation and docking experiments. MeroX 649 was run in RISEUP mode, with default crosslinker mass and fragmentation parameters for DSBU, 650 and in Quadratic mode with default crosslinker mass parameters for DMTMM; precursor mass range, $651300-10,000 \mathrm{Da}$; minimum precursor charge 4; precursor and fragment ion precisions 5.0 and 10.0 652 ppm, respectively; maximum number of missed cleavages 3; carbamidomethylation of cysteine and 653 oxidation of methionine, as fixed and variable modifications, respectively; results were filtered for 654 score $(>10)$ and false discovery rate, FDR $(<1 \%)$. FindXL was used to analyze and validate MeroX 655 results for DMTMM crosslinks. The default FindXL parameters were used as described before ${ }^{42}$ with 656 the possible crosslink amino acids adjustments for K, Y, S, or T on one peptide, and E or D on the 657 other peptide. MassAI was used to validate crosslinks identified by MeroX for DSBU with standard 
settings, except: 5 ppm MS accuracy, 0.05 Da MS/MS accuracy, 3 allowed missed cleavages, and

659 carbamidomethylation of cysteine and oxidation of methionine, as fixed and variable modifications, 660 respectively. Visualization of the crosslinks in the structures used Chimera ${ }^{74}$ with the Xlink Analyzer 661 plug-in ${ }^{75}$.

662

\section{Negative staining and cryo-EM sample preparation}

664 For negative staining, purified SCs were diluted to $0.01-0.05 \mathrm{mg} / \mathrm{mL}$ concentrations in TBS (50 mM 665 Tris-HCl, $\mathrm{pH} 7.4,150 \mathrm{mM} \mathrm{NaCl}, 0.01 \%$ DDM and $1 \mathrm{mM}$ AEBSF) buffer, $5 \mu \mathrm{L}$ applied to glow666 discharged (20 sec, 25 mA, Pelco easiGlow) carbon-coated Cu grids (CF300-CU, EMS), incubated 667 for 1 min and stained with $2 \%$ uranyl acetate. Grids were imaged on FEI Tecnai 12 transmission 668 electron microscope operating at $120 \mathrm{kV}$, with a CCD camera (Gatan BM-Ultrascan).

669 For cryo-EM, the tripartite SC was used as purified, whereas the purified bipartite SC was mixed 670 with a 5 -fold molar excess of purified cyt $c_{\mathrm{y}}{ }^{71} .2 .5 \mu \mathrm{L}$ of the mixture, containing $3-5 \mathrm{mg} / \mathrm{mL}$ proteins, 671 were applied to CFlat holey carbon grids (1.2/1.3-400 mesh or 2/2-300 mesh) (EMS), which were 672 glow discharged (2 min, $25 \mathrm{~mA}$, Pelco easiGlow) before sample application. Grids were blotted for $6739 \mathrm{sec}$ at force 0 (CFlat-1.2/1.3), or for $3 \mathrm{sec}$ at force -5 (CFlat-2/2), and flash-frozen in liquid ethane 674 cooled with liquid nitrogen using FEI Vitrobot Mark IV $\left(25^{\circ} \mathrm{C}, 100 \%\right.$ humidity). Plunge freezing 675 conditions were optimized using FEI Tecnai TF20 TEM operating at $200 \mathrm{kV}$, equipped with a FEI 676 Falcon II camera.

\section{Cryo-EM data acquisition and processing}

679 All cryo-EM grids were imaged using FEI Titan Krios electron microscope operating at $300 \mathrm{kV}$. 680 Images of the tripartite $\mathrm{SC}\left(\mathrm{SC} \sim c_{\mathrm{y}}\right)$ were recorded using a Gatan K2 Summit direct electron detector, 681 equipped with an energy quantum filter $(20 \mathrm{eV})$, and operated in super-resolution mode at a nominal 
682 magnification of $105,000 x$, resulting in a binned pixel size of $1.32 \AA$. Images were dose-fractionated

683 to 40 frames with a total exposure time of $10 \mathrm{sec}$, and a total dose of $40 \mathrm{e}^{-} / \mathrm{A}^{2}$. Automated data 684 acquisition was carried out using Latitude software (Gatan), and nominal defocus values varied from 6851 to $2.5 \mu \mathrm{m}$. Movies were motion corrected using MotionCor2 ${ }^{76}$ and CTF parameters were 686 determined with CTFFIND 4.1 ${ }^{77}$. About 10,000 particles were manually picked and subjected to an 687 initial reference-free 2D classification using Relion $3.0^{78}$. Representative classes were selected and 688 used as a template for auto-picking. After sorting and two rounds of 2D classification, $\sim 30,000$ 689 particles were retained from the dataset. For 3D classification, an initial model was created using $R$. 690 capsulatus $\mathrm{CIII}_{2}$ structure (PDB: 1ZRT) and low-pass filtered to $60 \AA$ using EMAN2 ${ }^{79}$. A 3D map 691 with a nominal resolution of $\sim 11 \AA$ containing a total of $\sim 12,000$ particles (40\%) was obtained, 692 showing a $\mathrm{CIII}_{2}$ associated on one side with a single copy of CIV. For further analyses, additional 693 datasets were collected using samples from the same batch and identical imaging conditions. From a 694 total of $\sim 17,000$ images, $\sim 1,000,000$ particles were automatically picked using the template obtained 695 from the first dataset, and after sorting and two rounds of $2 \mathrm{D}$ classification, $~ 500,000$ particles were 696 retained. Various subsets of all images were processed separately, and yielded the same overall results 697 with slightly different versions of individual maps. For clarity, only the paths to the best representative 698 of each of the final maps are shown (Fig. S2A, B and C). For 3D classification, the map obtained 699 from the first dataset (Fig. S2, Box 3) was low-pass filtered to $60 \AA$ and used as the initial model. Of 700 the five classes obtained, one showed clear features of a $\mathrm{CIII}_{2}$ dimer associated with a single monomer 701 of CIV (Fig. S2, class005 in A, class002 in B and C). The remaining classes showed the same overall 702 shape, but lacked density or resolution in different parts of the structure. Only two of the five maps 703 contained a second monomer of CIV associated with the other monomer of $\mathrm{CIII}_{2}$ (Fig. S2C). These 704 two classes showing this feature were combined, yielding a subset of $\sim 220,000$ particles. These 705 particles were further processed by another round of $2 \mathrm{D}$ classification, and subjected to 3D 
706 classification, yielding three classes (Fig. S2C). Only one of the maps obtained in this second round

707 of 3D classification clearly showed density for a second monomer of CIV. Aligned particles from this

708 group were subclassified into six classes in another round of 3D classification using a soft mask and

709 no image alignment. The low number of particles $(\sim 5,000)$ per class limited the resolution of the maps

$710(<10 \AA)$, and could not be further improved through 3D refinement.

711 Refinement of the individual maps (Fig. $\mathbf{S 2 A}, \mathbf{B})$ led to relatively low resolution reconstructions,

712 particularly in the CIV portion of the map, probably due to greater structural heterogeneity in the

713 sample with respect to this portion of the tripartite SC. The highest resolution was obtained with a

714 map containing a single copy of CIV, by combining all classes showing well defined features

715 corresponding to it and subjecting them to a second round of $3 \mathrm{D}$ classification, focused on CIV

716 portion of the map. For this purpose, a soft mask around CIV was created by fitting the closely related

717 structure of $P$. stutzeri (PDB: 3MK7) into the map and low-pass filtering it to $10 \AA$. All information

718 outside of this mask was subtracted from the aligned particles, and the remaining particles were

719 subjected to a masked classification without image alignment, to yield six classes (Fig. S2A).

720 Alternatively, a cylindrical mask was wrapped around the CIV portion of the map and used in a

721 similar procedure (Fig. S2B). In each case, classes showing the highest level of details were retained,

722 and the entire unsubtracted particles dataset subjected to 3D auto-refinement followed by per-particle

723 CTF refinement, Bayesian polishing and post-processing using Relion 3.0. The two extreme

724 conformations of the orientation of CIV relative to $\mathrm{CIII}_{2}$ were represented by the maps $\mathrm{SC}-1 \mathrm{~A}$ and

725 SC-1B (Fig. S2A,B), which were refined to $6.1 \AA$ and $7.2 \AA$ resolutions, respectively (Table S3).

726 Images of the bipartite $\mathrm{SC}$ supplemented with cyt $c_{\mathrm{y}}\left(\mathrm{SC}+c_{\mathrm{y}}\right)$ were recorded by a Gatan $\mathrm{K} 3$ direct

727 electron detector equipped with an energy quantum filter $(20 \mathrm{eV})$ and operated in counting mode at a

728 nominal magnification of $64,000 x$, corresponding to a pixel size of $1.36 \AA$. Images were dose-

729 fractionated to 80 frames with a total exposure time of $3.1 \mathrm{sec}$ and a total dose of $40 \mathrm{e} / \mathrm{A}^{2}$. Nominal 
730 defocus values varied from 1 to $2.5 \mu \mathrm{m}$. Automated data acquisition was carried out using Latitude

731 software (Gatan) and image shift $(\sim 2 \mu \mathrm{m}, 4$ images per stage position) was used for accelerated data

732 collection. For this sample 5,480 images were collected in the first session, motion corrected using

733 MotionCor $2{ }^{76}$, and CTF parameters were determined with CTFFIND $4.1^{77}$. For auto-picking, the

734 template previously obtained with the tripartite SC was used, with this and all subsequent steps done

735 in Relion $3.0^{78}$. After sorting and two rounds of 2D classification, $\sim 228,000$ particles were retained

736 (Fig. S5A). The same initial model as for the tripartite SC was used for 3D classification into five

737 classes. Two classes (001 and 002) resembled more to the overall shape of a $\mathrm{CIII}_{2}$ without CIV, while

738 the other three classes showed the same overall shape of a $\mathrm{CIII}_{2} \mathrm{CIV} \mathrm{SC}$, as seen with the tripartite

739 sample. 37,460 particles corresponding to the SC class with the highest level of detail were combined

740 with SC particles from the second dataset (Fig. S5B) to yield the best final map of the bipartite SC.

741 The second dataset consisted of 12,200 images which were collected and processed under the same

742 conditions as the first dataset. 1.6 million particles were auto-picked and after two rounds of 2D

743 classification, classes were split into $\sim 340,000$ large particles likely representing $\mathrm{CIII}_{2} \mathrm{CIV}$ and

$744 \sim 465,000$ particles resembling $\mathrm{CIII}_{2}$ (Fig. S5B,D). The former particles were subjected to 3D

745 classification into three classes, and the class most similar to the overall shape of $\mathrm{CIII}_{2} \mathrm{CIV}$ was

746 identified. It contained $\sim 118.000$ particles, but due to its low level of detail, it was subjected to another

747 round of 2D classification. The 34,819 particles thus retained were combined with 37,460 SC particles

748 from the first dataset (Fig. S5A). After 3D classification into five classes, the class with the highest

749 level of detail contained $56 \%$ of particles, and clearly showed the overall shape of $\mathrm{CIII}_{2} \mathrm{CIV} .41,017$

750 aligned particles corresponding to this class were extracted and subjected to a second round of 3D

751 classification without image alignment using a soft mask. Of the six 3D classes, the one with the

752 highest nominal resolution contained 14,978 particles (36\%) and was subjected to 3D auto-refinement

753 and post processing, followed by per-particle CTF refinement and Bayesian polishing. After a second 
754 round of 3D auto-refinement and post processing, the final map (SC-2A) of the bipartite $\mathrm{CIII}_{2} \mathrm{CIV}$ 755 was obtained at a nominal resolution of $5.18 \AA$ A (Table S3). This map was very similar to that (SC-

756 1A) of the tripartite $\mathrm{CIII}_{2} \mathrm{CIV}$, except for its higher resolution and strongly improved density and

757 resolution of the extra TMHs at the interface. Unlike the tripartite samples, no major class 758 corresponding to the conformation seen in SC-1B was identified.

759 As the bipartite $\mathrm{SC}$ samples contained a significant amount of $\mathrm{CIII}_{2}$ particles without CIV, subsets

760 of smaller 2D classes consistent with a $\mathrm{CIII}_{2}$ dimer were selected and processed separately. Of the

$761 \sim 376,000$ total particles retained from the dataset 1, 267,000 were identified as resembling $\mathrm{CIII}_{2}$.

762 After a second round of 2D classification (Fig. S5C), 213,000 particles were retained and used to

763 generate an $a b$ initio 3D model. This model was lowpass filtered to $60 \AA$ and used as initial model

764 for the 3D classification of $\sim 465,000 \mathrm{CIII}_{2}$ particles from dataset 2 (Fig. S5D). Particles corresponding

765 to the class with the highest resolution and level of detail $(\sim 185,000)$ were combined with the $\mathrm{CIII}_{2}$

766 particles from dataset 1 to yield $\sim 400,000$ particles, and subjected to $3 \mathrm{D}$ classification. The $\sim 170,000$

767 particles corresponding to the best class were further processed following two different strategies.

$768 \mathrm{CIII}_{2}$ being a homodimer based on X-ray structures, $\mathrm{C} 2$ symmetry was applied in the next round of

769 3D classification and in all subsequent steps (Fig. S5E). The best class contained $\sim 120,000$ particles

770 (70\%) which were extracted and, similar to $\mathrm{CIII}_{2} \mathrm{CIV}$, subjected to another round of 3D classification

771 without image alignment using a soft mask. Of the six classes obtained, some showed the external

772 domain (ED) of both monomers of the FeS protein close to heme $b_{\mathrm{L}}$ ("b position", b-b) while other

773 classes showed both EDs close to heme $c_{1}$ (“c position", c-c). Individual classes were subjected to 3D

774 auto-refinement and post processing, followed by two rounds of per-particle CTF refinement,

775 Bayesian polishing, 3D auto-refinement and post processing. Only the final map with the highest

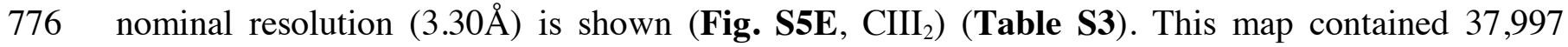

777 particles and showed both FeS-EDs close to $b$ position, but at a lower local resolution and occupancy 
than the rest of the map, indicating structural flexibility and conformational heterogeneity. A similar

779 approach was used in Fig. S5F, except that no C2 symmetry was applied in the two rounds of 3D

780 classifications. Of the three best classes obtained in the second round, one showed both EDs in the b

781 position, one showed both in the c position, and one showed a heterodimeric conformation with one

782 monomer in the $\mathrm{b}$ and the other in the $\mathbf{c}$ position. Each of the maps was further processed as in Fig.

783 S5E, but C2 symmetry was only applied in case of the homodimeric structures. The final maps were

$784 \mathrm{CIII}_{2} \mathrm{c}-\mathrm{c}$ with both ED's in the c position at $3.8 \AA \mathrm{AIII}_{2} \mathrm{~b}$-b with both ED's in the b position at $3.5 \AA$,

785 and $\mathrm{CIII}_{2}$ b-c with one ED in the $\mathrm{b}$ and the second in the c position at $4.2 \AA$ (Table S3). The nominal

786 resolutions thus obtained were slightly lower than in map $\mathrm{CIII}_{2}$ (Fig. S5E). However, by omitting C2

787 symmetry application to the $3 \mathrm{D}$ classifications, the conformational heterogeneity of the FeS protein

788 EDs was resolved, and a subset of particles showing a heterodimeric conformation was identified.

789 The different locations (i.e., b or c positions) of the ED's were clear in the three maps (Fig. S5F) but

790 their occupancy and local resolutions was lower than the rest of the structure.

791 A third dataset was also collected and yielded $\sim 303,000$ particles after the first 2D classification.

792 Adding these particles to the first two datasets did not improve the maps shown in Fig. S5A,B, but it

793 turned out to be informative. As shown in Fig. S5G, particles from dataset 3 were subjected to two

794 rounds of 3D classification. Interestingly, an extra density near the periplasmic domain of CcoP, could

795 be seen in some classes. This was not observed in any subclass obtained from the datasets 1 and 2 ,

796 and tentatively thought to correspond to the cyt $c$ domain of cyt $c_{\mathrm{y}}$. Focused classification followed

797 by $3 \mathrm{D}$ auto-refinement and post processing, using wider soft masks around the periplasmic domain

798 of CcoP to avoid cutting-off any of the extra density, led to a final map (SC-2B) with limited

799 resolution $(10.5 \AA)$, and could not be further improved by sub-classification due to the low number of 800 particles in each subclass.

801 
803 The X-ray based structure of $R$. capsulatus CIII $_{2}$ (PDB: 1ZRT) was fitted into map CIII (EMD- $^{\text {(EM) }}$

804 22189) which had the highest nominal resolution of all maps obtained (Fig. S5E), and refined using

805 Phenix $1.16^{80}$. The real space refinement approach included four rounds of global minimization, local

806 grid search, morphing and simulated annealing, with the final round also including ADP (B-factor)

807 refinement. Each round included 5 cycles using default settings, and morphing and annealing were

808 performed in each cycle. Secondary structures were determined by Phenix1.16, using default search

809 settings and restrictions were applied during real space refinement. Due to the low occupancy and

810 limited local resolution, the FeS-ED proteins (residues 50-191) were only subjected to rigid body

811 fitting followed by two rounds of global minimization and local grid search (5 cycles each), but not

812 to morphing and simulated annealing. To ensure the correct cofactor geometry, hemes and [2Fe-2S]

813 clusters including their coordinating residues were copied from the high-resolution X-ray structure of

814 the homologous $\mathrm{CIII}_{2}$ (cyt $b c_{1}$ ) from $R$. sphaeroides (PDB: 6NHH). Validation was performed using

815 MolProbity ${ }^{81}$ (http://molprobity.biochem.duke.edu/), and outliers (Ramachandran, rotamer, bonds,

816 angles) were manually corrected in $\operatorname{Coot}^{82}$, using real space refinement and regularization. The model

817 that was refined in map $\mathrm{CIII}_{2}$ (EMD-22189) was subsequently used for rigid body fitting into the

818 maps $\mathrm{CIII}_{2}$ b-b, $\mathrm{CIII}_{2}$ c-c and $\mathrm{CIII}_{2}$ b-c (Fig. S5F) (Table S3). Each chain was treated as one separate

819 body, except the FeS protein, which was split into its TMH (11 to 49) and ED (50 to 191) residues.

820 After the procedure, the linker region between the ED and TMH (residues 40-50) was remodeled in

821 Coot using real space refinement and regularization.

822

823 Structural modeling of $R$. capsulatus cbb $_{3}$-type CIV subunits, CcoH and cyt $c_{\mathrm{y}}$

824 Modeling of $\boldsymbol{R}$. capsulatus $\boldsymbol{c} \boldsymbol{b} \boldsymbol{b}_{3}$-type CIV. The Pseudomonas stutzeri c $_{\text {- }} b_{3}$-type CIV structure (PDB:

825 5DJQ) (sequence identities for $R$. capsulatus CcoN: 68\%, CcoO: $55 \%$, CcoP: 34\%) was used as a 
826 template, and comparative models were computed using MODELLER v9.18 ${ }^{83}$ with defined

827 secondary structure and crosslinking restraints. The secondary structures for the regions without any 828 template coverage (CcoP residues 104 to 112,176 to 178,184 to 197 and 204 to 215; CcoO residues

829172 to 185 and 192 to 199$)$, due to the insertion sequences that are present only in $R$. capsulatus CIV,

830 were estimated using PsiPred Protein Sequence Analysis Workbench ${ }^{84,85}$, and added as restraints to

831 MODELLER. Cross-link distances were added as Gaussian restraints to MODELLER with a mean

832 of $18.0 \AA$ and a standard deviation of $1.0 \AA$. Problematic loop regions (a total of ten loops longer than

833 four amino acids) were detected by MolProbity ${ }^{81,86}$ and remodeled using MODELLER "slow"

834 refinement method (Table S3). As $R$. capsulatus $\mathrm{CcoN}$ is longer than that of $P$. stutzeri with a

835 predicted extra N-ter TMH, whereas CcoP is shorter with only one predicted N-ter TMH instead of

836 two, these regions were not included into the model. The regions CcoO 179-214 as well as CcoP 1-

$83712,53-59,161-173$ and 272-280 without template coverage that were not supported by the cryo-EM

838 maps were omitted.

839 Modeling of $\mathbf{C c o H}$ and cyt $\boldsymbol{c}$ domain of $\boldsymbol{c}_{\mathbf{y}}$. For $\mathrm{CcoH}$ (residues Met 1 to Thr35) five ab initio 840 models were obtained using I-TASSER server ${ }^{87}$. All models were almost identical, and one with the

841 lowest energy score was retained. For $R$. capsulatus cyt $c_{\mathrm{y}}$, its homologs with known structures were 842 detected using HHpred ${ }^{88}$, and the structure of cyt $c$ domain of cyt $c_{552}$ from Paracoccus denitirificans

843 (PDB: 3M97; sequence identity: 61\%) was used as a template for the soluble cyt $c$ domain of $c_{\mathrm{y}}$ and 844 comparative models were computed using MODELLER ${ }^{83}$.

845 Modeling of the extra N-ter TMH of CcoN (TMH0). A 29-residue model consisting of CcoN 846 residues Arg25 to Asp53, which includes the predicted transmembrane region (Met30-Leu48, 847 UniProtKB, D5ARP4), was obtained from the I-TASSER server ${ }^{89}$. The alpha helical region included 848 residues Leu27 to Thr50, and the model including the residues Arg25 to Leu48 was manually fitted 849 into the density map for visualization purpose in Fig. 2. Corresponding coordinates were not 
850 deposited in the PDB because the registration could not be determined due to the lack of side chain 851 density.

852 Modeling of cyt $\boldsymbol{c}_{\mathbf{y}}$ and CcoH TMHs interactions. The 30 residues long cyt $c_{\mathrm{y}} \mathrm{TMH}$ was

853 manually docked into the density map by positioning Phe15 and Tyr21 into the corresponding 854 densities, and refined by real-space refinement and regularization using Coot. The I-TASSER model 855 of $\mathrm{CcoH}$ was docked into the map by moving it along the corresponding density, retaining its close 856 association with cyt $c_{y}$ TMH. The best fit was found when Ala23 and Val24 of CcoH were located at 857 the interface with cyt $c_{y}$ TMH. The interaction of the two helices was optimized by GalaxyWEB ${ }^{90}$, 858 and the model with the lowest energy profile was refined in map SC-2A using Phenix 1.17. The 859 refinement strategy included 5 cycles of global minimization, rigid body fitting (where cyt $c_{\mathrm{y}}$ and

$860 \mathrm{CcoH}$ chains were treated as separate bodies), local grid search and ADP (B-factor) refinement with 861 default settings for all steps. Secondary structure restrictions were applied to the $\alpha$-helical parts of $862 \mathrm{CcoH}$ as predicted by I-TASSER (residues 12 to 34) and to cyt $c_{\mathrm{y}}$ as predicted by GalaxyWEB 863 (residues 2 to 9 and 12 to 29). $\mathrm{CIII}_{2}$ and $\mathrm{CIV}$ models were present during the refinement to keep the 864 rest of the map occupied, but changes made during this procedure were discarded as these models 865 were refined separately.

866 Integrative modeling and docking $\mathbf{C c o H}$, cyt $\boldsymbol{c}_{\mathbf{y}}$ and cyt $\boldsymbol{c}_{2}$ to bipartite SC. The entire SC was 867 assembled by an integrative modeling approach using the cryo-EM map, XL-MS, co-evolutionary 868 analysis, and subunit models described above. $\mathrm{CIII}_{2}$ and CIV models were fitted into the cryo-EM 869 maps in UCSF Chimera ${ }^{74}$. Additional data about the interaction interfaces between the different 870 subunits were obtained using RaptorX-ComplexContact ${ }^{33}$ for each pair of the SC subunits. Based on 871 co-evolution and machine learning, this method predicted the pairs of residues that are in contact. The 872 PatchDock, which is an efficient rigid docking method that maximizes geometric shape 873 complementarity ${ }^{34}$, was used to generate docked configurations of $\mathrm{CcoH}$, cyt $c_{2}$ and cyt $c_{\mathrm{y}}$ to $\mathrm{CIII}_{2}$ 
874 and CIV , as appropriate. The different subunits were docked in parallel and independently from each 875 other.

876 Docking of $\mathbf{C c o H}$ to CIV. A total of 16 contacts predicted by RaptorX-ComplexContact, with 877 probabilities higher than 0.5 , were used as distance restraints for protein-protein docking ${ }^{34}$. The 878 models satisfied 13 of the contacts, and a single cluster of docked models evidenced by the 879 convergence of the top 100 results, was obtained (Fig. S7). This cluster coincided with the additional 880 feature seen at the SC interface, and CcoH TMH was modelled into this feature.

881 Docking of cyt $\boldsymbol{c}_{2}$ to $\mathbf{C I I I}_{2}$ and CIV. $R$. capsulatus cyt $c_{2}$ of known structure (PDB: $1 \mathrm{C} 2 \mathrm{~N}$ ) was 882 docked to $\mathrm{CIII}_{2}$ using the three distance restraints between cyt $c_{2}$ and cyt $c_{1}$ derived from the protein 883 cross-linking data (Table S4). Similarly, cyt $c_{2}$ was docked to CIV using nine distance restraints 884 provided byXLs ( 1 crosslink to $\mathrm{CcoP}$ and 8 to $\mathrm{CcoO}$, Table S4). The models yielded one main cluster 885 in each case, and covered $100 \%$ and $89 \%$ of the data for $\mathrm{CIII}_{2}$ and CIV, respectively.

886 Docking of cyt $\boldsymbol{c}_{\mathrm{y}}$ to CIII $_{2}$ CIV SC. The model of cyt $c$ domain of $c_{\mathrm{y}}$ was docked by PatchDock 887 to bipartite $\mathrm{CIII}_{2} \mathrm{CIV}$, which has both $\mathrm{FeS}$-EDs of $\mathrm{CIII}_{2}$ in the b position. Ten distance restraints 888 derived from the XL-MS data (6 with DMTMM and 4 with DSBU, Table S4) were used, yielding 889 two main clusters (1 and 2) of the docked models on the $p$ side of $\mathrm{CIII}_{2}$ (Fig. S4), and satisfied 100\% 890 of the restraints. As the cryo-EM data revealed that $\mathrm{CIII}_{2}$ particles could have their FeS-EDs in the c 891 position, the corresponding $\mathrm{CIII}_{2} \mathrm{c}-\mathrm{c}, \mathrm{CIII}_{2}$ b-b and $\mathrm{CIII}_{2}$ b-c models were used for docking via 892 Patchdock the cyt $c$ domain of $c_{\mathrm{y}}$ onto $\mathrm{CIII}_{2}$.

893 All the models were ranked using statistically optimized atomic potentials (SOAP) ${ }^{91}$, and those 894 that have low SOAP scores were retained. 


\section{References}

8971 Nicholls, D. G. \& Ferguson, S. J. Bioenergetics 4. (Elsevier, 2013).

8982 Darrouzet, E., Moser, C. C., Dutton, P. L. \& Daldal, F. Large scale domain movement in $899 \quad$ cytochrome $b c_{1}$ : a new device for electron transfer in proteins. Trends Biochem Sci 26, 445-451, doi:10.1016/s0968-0004(01)01897-7 (2001).

3 Esser, L. et al. Surface-modulated motion switch: capture and release of iron-sulfur protein in the cytochrome $b c_{1}$ complex. Proc Natl Acad Sci $U \quad S \quad A \quad \mathbf{1 0 3 ,}$ 13045-13050, doi:10.1073/pnas.0601149103 (2006).

4 Khalfaoui-Hassani, B. et al. in Cytochrome Complexes: Evolution, Structures, Energy Transduction, and Signaling. (eds W. Cramer \& T. Kallas) 527-555 (Springer, 2016).

5 Smith, M. A., Finel, M., Korolik, V. \& Mendz, G. L. Characteristics of the aerobic respiratory chains of the microaerophiles Campylobacter jejuni and Helicobacter pylori. Arch Microbiol 174, 1-10, doi:10.1007/s002030000174 (2000).

6 Aspholm, M. et al. Structural alterations in a component of cytochrome $c$ oxidase and molecular evolution of pathogenic Neisseria in humans. PLoS Pathog 6, e1001055, doi:10.1371/journal.ppat.1001055 (2010).

7 Ekici, S., Pawlik, G., Lohmeyer, E., Koch, H. G. \& Daldal, F. Biogenesis of cbb(3)-type cytochrome c oxidase in Rhodobacter capsulatus. Biochim Biophys Acta 1817, 898-910, doi:10.1016/j.bbabio.2011.10.011 (2012).

8 Jenney, F. E., Jr. \& Daldal, F. A novel membrane-associated $c$-type cytochrome, cyt $c_{\mathrm{y}}$, can mediate the photosynthetic growth of Rhodobacter capsulatus and Rhodobacter sphaeroides. EMBO J 12, 1283-1292 (1993).

9 Turba, A., Jetzek, M. \& Ludwig, B. Purification of Paracoccus denitrificans cytochrome $c_{552}$ and sequence analysis of the gene. Eur J Biochem 231, 259-265 (1995).

10 Albert, I., Rutherford, A. W., Grav, H., Kellermann, J. \& Michel, H. The 18 kDa cytochrome $c_{553}$ from Heliobacterium gestii: gene sequence and characterization of the mature protein. Biochemistry 37, 9001-9008, doi:10.1021/bi9731347 (1998).

11 Kim, M. S. et al. Isolation and Characterization of a Hybrid Respiratory Supercomplex Consisting of Mycobacterium tuberculosis Cytochrome bcc and Mycobacterium smegmatis Cytochrome $a a_{3}$. J Biol Chem 290, 14350-14360, doi:10.1074/jbc.M114.624312 (2015).

12 Winstedt, L. \& von Wachenfeldt, C. Terminal oxidases of Bacillus subtilis strain 168: one quinol oxidase, cytochrome $a a_{3}$ or cytochrome $b d$, is required for aerobic growth. $J$ Bacteriol 182, 65576564, doi:10.1128/jb.182.23.6557-6564.2000 (2000).

13 Hochkoeppler, A., Jenney, F. E., Jr., Lang, S. E., Zannoni, D. \& Daldal, F. Membrane-associated cytochrome $c_{y}$ of Rhodobacter capsulatus is an electron carrier from the cytochrome $b c_{1}$ complex to the cytochrome $c$ oxidase during respiration. $J$ Bacteriol 177, 608-613, doi:10.1128/jb.177.3.608-613.1995 (1995).

14 Enriquez, J. A. Supramolecular Organization of Respiratory Complexes. Annu Rev Physiol 78, 533-561, doi:10.1146/annurev-physiol-021115-105031 (2016).

15 Melo, A. M. \& Teixeira, M. Supramolecular organization of bacterial aerobic respiratory chains: From cells and back. Biochim Biophys Acta 1857, 190-197, doi:10.1016/j.bbabio.2015.11.001 (2016).

16 Brzezinski, P. New Structures Reveal Interaction Dynamics in Respiratory Supercomplexes. Trends Biochem Sci, doi:10.1016/j.tibs.2019.10.011 (2019). 
940

941

942

943

944

945

946

947

948

949

950

951

952

953

954

955

956

957

958

959

960

961

962

963

964

965

966

967

968

969

970

971

972

973

974

975

976

977

978

979

980

981

982

983

984

985

986

17 Letts, J. A., Fiedorczuk, K., Degliesposti, G., Skehel, M. \& Sazanov, L. A. Structures of Respiratory Supercomplex I+III2 Reveal Functional and Conformational Crosstalk. Mol Cell 75, 1131-1146 e1136, doi:10.1016/j.molcel.2019.07.022 (2019).

18 Quintana-Cabrera, R. \& Soriano, M. E. ER Stress Priming of Mitochondrial Respiratory suPERKomplex Assembly. Trends Endocrinol Metab 30, 685-687, doi:10.1016/j.tem.2019.08.003 (2019).

19 Letts, J. A., Fiedorczuk, K. \& Sazanov, L. A. The architecture of respiratory supercomplexes. Nature 537, 644-648, doi:10.1038/nature19774 (2016).

$20 \mathrm{Gu}$, J. et al. The architecture of the mammalian respirasome. Nature 537, 639-643, doi:10.1038/nature19359 (2016).

21 Hartley, A. M. et al. Structure of yeast cytochrome $c$ oxidase in a supercomplex with cytochrome $b c_{1}$. Nat Struct Mol Biol 26, 78-83, doi:10.1038/s41594-018-0172-z (2019).

22 Berry, E. A. \& Trumpower, B. L. Isolation of ubiquinol oxidase from Paracoccus denitrificans and resolution into cytochrome $b c_{1}$ and cytochrome $c-a a_{3}$ complexes. J Biol Chem 260, 24582467 (1985).

23 Kao, W. C. et al. The obligate respiratory supercomplex from Actinobacteria. Biochim Biophys Acta 1857, 1705-1714, doi:10.1016/j.bbabio.2016.07.009 (2016).

24 Gong, H. et al. An electron transfer path connects subunits of a mycobacterial respiratory supercomplex. Science 362, doi:10.1126/science.aat8923 (2018).

25 Wiseman, B. et al. Structure of a functional obligate complex $\mathrm{III}_{2} \mathrm{IV}_{2}$ respiratory supercomplex from Mycobacterium smegmatis. Nat Struct Mol Biol 25, 1128-1136, doi:10.1038/s41594-0180160-3 (2018).

26 Ducluzeau, A. L., Ouchane, S. \& Nitschke, W. The $c b b_{3}$ oxidases are an ancient innovation of the domain bacteria. Mol Biol Evol 25, 1158-1166, doi:10.1093/molbev/msn062 (2008).

27 Myllykallio, H., Drepper, F., Mathis, P. \& Daldal, F. Electron-transfer supercomplexes in photosynthesis and respiration. Trends Microbiol 8, 493-494, doi:10.1016/s0966-842x(00)01831$\mathrm{x}(2000)$.

28 Davidson, E., Ohnishi, T., Tokito, M. \& Daldal, F. Rhodobacter capsulatus mutants lacking the Rieske FeS protein form a stable cytochrome $b c_{1}$ subcomplex with an intact quinone reduction site. Biochemistry 31, 3351-3358, doi:10.1021/bi00128a007 (1992).

29 Kulajta, C., Thumfart, J. O., Haid, S., Daldal, F. \& Koch, H. G. Multi-step assembly pathway of the $c b b_{3}$-type cytochrome $c$ oxidase complex. J Mol Biol 355, 989-1004, doi:10.1016/j.jmb.2005.11.039 (2006).

30 Valkova-Valchanova, M. B., Saribas, A. S., Gibney, B. R., Dutton, P. L. \& Daldal, F. Isolation and characterization of a two-subunit cytochrome $b$ - $c_{1}$ subcomplex from Rhodobacter capsulatus and reconstitution of its ubihydroquinone oxidation $\left(\mathrm{Q}_{\mathrm{o}}\right)$ site with purified Fe-S protein subunit. Biochemistry 37, 16242-16251, doi:10.1021/bi981651z (1998).

31 Gray, K. A. et al. Rhodobacter capsulatus contains a novel $c b$-type cytochrome $c$ oxidase without $\mathrm{a} \mathrm{Cu}_{\mathrm{A}}$ center. Biochemistry 33, 3120-3127, doi:10.1021/bi00176a047 (1994).

32 Ozturk, Y. et al. Soluble variants of Rhodobacter capsulatus membrane-anchored cytochrome $c_{\mathrm{y}}$ are efficient photosynthetic electron carriers. J Biol Chem 283, 13964-13972, doi:10.1074/jbc.M800090200 (2008).

33 Zeng, H. et al. ComplexContact: a web server for inter-protein contact prediction using deep learning. Nucleic Acids Res 46, W432-W437, doi:10.1093/nar/gky420 (2018).

34 Schneidman-Duhovny, D., Inbar, Y., Nussinov, R. \& Wolfson, H. J. PatchDock and SymmDock: servers for rigid and symmetric docking. Nucleic Acids Res 33, W363-367, doi:10.1093/nar/gki481 (2005). 
987

988

989

990

991

992

993

994

995

996

997

998

999

1000

1001

1002

1003

1004

1005

1006

1007

1008

1009

1010

1011

1012

1013

1014

1015

1016

1017

1018

1019

1020

1021

1022

1023

1024

1025

1026

1027

1028

1029

1030

1031

1032

1033
35 Pawlik, G. et al. The putative assembly factor $\mathrm{CcoH}$ is stably associated with the $c b b_{3}$-type cytochrome oxidase. J Bacteriol 192, 6378-6389, doi:10.1128/JB.00988-10 (2010).

36 Covian, R. \& Trumpower, B. L. Rapid electron transfer between monomers when the cytochrome $b c_{1}$ complex dimer is reduced through center N. J Biol Chem 280, 22732-22740, doi:10.1074/jbc.M413592200 (2005).

37 Castellani, M. et al. Direct demonstration of half-of-the-sites reactivity in the dimeric cytochrome $b c_{1}$ complex: enzyme with one inactive monomer is fully active but unable to activate the second ubiquinol oxidation site in response to ligand binding at the ubiquinone reduction site. $J$ Biol Chem 285, 502-510, doi:10.1074/jbc.M109.072959 (2010).

38 Cooley, J. W., Lee, D. W. \& Daldal, F. Across membrane communication between the $\mathrm{Q}_{o}$ and $\mathrm{Q}_{\mathrm{i}}$ active sites of cytochrome $b c_{1}$. Biochemistry 48, 1888-1899, doi:10.1021/bi802216h (2009).

39 Slavin, M. \& Kalisman, N. Structural Analysis of Protein Complexes by Cross-Linking and Mass Spectrometry. Methods Mol Biol 1764, 173-183, doi:10.1007/978-1-4939-7759-8_11 (2018).

40 Gotze, M. et al. Automated assignment of MS/MS cleavable cross-links in protein 3D-structure analysis. J Am Soc Mass Spectrom 26, 83-97, doi:10.1007/s13361-014-1001-1 (2015).

41 Solmaz, S. R. \& Hunte, C. Structure of complex III with bound cytochrome $c$ in reduced state and definition of a minimal core interface for electron transfer. J Biol Chem 283, 17542-17549, doi:10.1074/jbc.M710126200 (2008).

42 Kalisman, N., Adams, C. M. \& Levitt, M. Subunit order of eukaryotic TRiC/CCT chaperonin by cross-linking, mass spectrometry, and combinatorial homology modeling. Proc Natl Acad Sci U S A 109, 2884-2889, doi:10.1073/pnas.1119472109 (2012).

43 Iacobucci, C. et al. A cross-linking/mass spectrometry workflow based on MS-cleavable crosslinkers and the MeroX software for studying protein structures and protein-protein interactions. Nat Protoc 13, 2864-2889, doi:10.1038/s41596-018-0068-8 (2018).

44 Osyczka, A., Moser, C. C. \& Dutton, P. L. Fixing the Q cycle. Trends Biochem Sci 30, 176-182, doi:10.1016/j.tibs.2005.02.001 (2005).

45 Crofts, A. R. et al. The Q-cycle reviewed: How well does a monomeric mechanism of the bc(1) complex account for the function of a dimeric complex? Biochim Biophys Acta 1777, 1001-1019, doi:10.1016/j.bbabio.2008.04.037 (2008).

46 Buschmann, S. et al. The structure of $c b b_{3}$ cytochrome oxidase provides insights into proton pumping. Science 329, 327-330, doi:10.1126/science.1187303 (2010).

47 Myllykallio, H., Drepper, F., Mathis, P. \& Daldal, F. Membrane-anchored cytochrome $c_{\mathrm{y}}$ mediated microsecond time range electron transfer from the cytochrome $b c_{1}$ complex to the reaction center in Rhodobacter capsulatus. Biochemistry 37, 5501-5510, doi:10.1021/bi973123d (1998).

48 Lee, D. W., Ozturk, Y., Osyczka, A., Cooley, J. W. \& Daldal, F. Cytochrome $b c_{1}-c_{y}$ fusion complexes reveal the distance constraints for functional electron transfer between photosynthesis components. J Biol Chem 283, 13973-13982, doi:10.1074/jbc.M800091200 (2008).

49 Berry, E. A. et al. X-Ray Structure of Rhodobacter capsulatus Cytochrome $b c_{1}$ : Comparison with its Mitochondrial and Chloroplast Counterparts. Photosynth Res 81, 251-275, doi:10.1023/B:PRES.0000036888.18223.0e (2004).

$50 \mathrm{Xia}$, D. et al. The road to the crystal structure of the cytochrome $\mathrm{bc}_{1}$ complex from the anoxigenic, photosynthetic bacterium Rhodobacter sphaeroides. J Bioenerg Biomembr 40, 485-492, doi:10.1007/s10863-008-9180-8 (2008).

51 Esser, L. et al. Inhibitor-complexed structures of the cytochrome $b c_{1}$ from the photosynthetic bacterium Rhodobacter sphaeroides. J Biol Chem 283, 2846-2857, doi:10.1074/jbc.M708608200 (2008). 
1034

1035

1036

1037

1038

1039

1040

1041

1042

1043

1044

1045

1046

1047

1048

1049

1050

1051

1052

1053

1054

1055

1056

1057

1058

1059

1060

1061

1062

1063

1064

1065

1066

1067

1068

1069

1070

1071

1072

1073

1074

1075

1076

1077

1078

52 Sousa, J. S., Mills, D. J., Vonck, J. \& Kuhlbrandt, W. Functional asymmetry and electron flow in the bovine respirasome. Elife 5, doi:10.7554/eLife.21290 (2016).

53 Swierczek, M. et al. An electronic bus bar lies in the core of cytochrome $b c_{1}$. Science 329, 451454, doi:10.1126/science.1190899 (2010).

54 Lanciano, P., Lee, D. W., Yang, H., Darrouzet, E. \& Daldal, F. Intermonomer electron transfer between the low-potential $b$ hemes of cytochrome $b c_{1}$. Biochemistry 50, 1651-1663, doi:10.1021/bi101736v (2011).

55 Moser, C. C., Keske, J. M., Warncke, K., Farid, R. S. \& Dutton, P. L. Nature of biological electron transfer. Nature 355, 796-802, doi:10.1038/355796a0 (1992).

56 Wikstrom, M., Krab, K. \& Sharma, V. Oxygen Activation and Energy Conservation by Cytochrome $c$ Oxidase. Chem Rev 118, 2469-2490, doi:10.1021/acs.chemrev.7b00664 (2018).

57 Brzezinski, P. \& Gennis, R. B. Cytochrome $c$ oxidase: exciting progress and remaining mysteries. J Bioenerg Biomembr 40, 521-531, doi:10.1007/s10863-008-9181-7 (2008).

58 Verissimo, A. F., Sousa, F. L., Baptista, A. M., Teixeira, M. \& Pereira, M. M. Thermodynamic redox behavior of the heme centers of $c b b_{3}$ heme-copper oxygen reductase from Bradyrhizobium japonicum. Biochemistry 46, 13245-13253, doi:10.1021/bi700733g (2007).

59 Otten, M. F. et al. Cytochromes $c_{550}, c_{552}$, and $c_{1}$ in the electron transport network of Paracoccus denitrificans: redundant or subtly different in function? J Bacteriol 183, 7017-7026, doi:10.1128/JB.183.24.7017-7026.2001 (2001).

60 Kappler, U. \& Dahl, C. Enzymology and molecular biology of prokaryotic sulfite oxidation. FEMS Microbiol Lett 203, 1-9, doi:10.1111/j.1574-6968.2001.tb10813.x (2001).

61 Khalfaoui-Hassani, B. et al. Widespread Distribution and Functional Specificity of the Copper Importer CcoA: Distinct $\mathrm{Cu}$ Uptake Routes for Bacterial Cytochrome $c$ Oxidases. MBio 9, doi:10.1128/mBio.00065-18 (2018).

62 Wittig, I., Braun, H. P. \& Schagger, H. Blue native PAGE. Nat Protoc 1, 418-428, doi:10.1038/nprot.2006.62 (2006).

63 Laemmli, U. K. Cleavage of structural proteins during the assembly of the head of bacteriophage T4. Nature 227, 680-685, doi:10.1038/227680a0 (1970).

64 Bartsch, H., Arndt, C., Koristka, S., Cartellieri, M. \& Bachmann, M. Silver staining techniques of polyacrylamide gels. Methods Mol Biol 869, 481-486, doi:10.1007/978-1-61779-821-4_42 (2012).

65 Yan, L. J. \& Forster, M. J. Resolving mitochondrial protein complexes using nongradient blue native polyacrylamide gel electrophoresis. Anal Biochem 389, 143-149, doi:10.1016/j.ab.2009.03.043 (2009).

66 Thomas, P. E., Ryan, D. \& Levin, W. An improved staining procedure for the detection of the peroxidase activity of cytochrome P-450 on sodium dodecyl sulfate polyacrylamide gels. Anal Biochem 75, 168-176, doi:10.1016/0003-2697(76)90067-1 (1976).

67 Gill, S. C. \& von Hippel, P. H. Calculation of protein extinction coefficients from amino acid sequence data. Anal. Biochem. 182, 319-326 (1989).

68 Atta-Asafo-Adjei, E. \& Daldal, F. Size of the amino acid side chain at position 158 of cytochrome $b$ is critical for an active cytochrome $b c_{1}$ complex and for photosynthetic growth of Rhodobacter capsulatus. Proc Natl Acad Sci U S A 88, 492-496, doi:10.1073/pnas.88.2.492 (1991).

69 Peters, A., Kulajta, C., Pawlik, G., Daldal, F. \& Koch, H. G. Stability of the $c b b_{3}$-type cytochrome oxidase requires specific CcoQ-CcoP interactions. $J$ Bacteriol 190, 5576-5586, doi:10.1128/JB.00534-08 (2008). 
1079

1080

1081

1082

1083

1084

1085

1086

1087

1088

1089

1090

1091

1092

1093

1094

1095

1096

1097

1098

1099

1100

1101

1102

1103

1104

1105

1106

1107

1108

1109

1110

1111

1112

1113

1114

1115

1116

1117

1118

1119

1120

1121

1122

1123

1124

1125

70 Selamoglu, N. et al. Comparative differential cuproproteomes of Rhodobacter capsulatus reveal novel copper homeostasis related proteins. Metallomics 12, 572-591, doi:10.1039/c9mt00314b (2020).

71 Myllykallio, H., Jenney, F. E., Jr., Moomaw, C. R., Slaughter, C. A. \& Daldal, F. Cytochrome $c_{\mathrm{y}}$ of Rhodobacter capsulatus is attached to the cytoplasmic membrane by an uncleaved signal sequence-like anchor. J Bacteriol 179, 2623-2631, doi:10.1128/jb.179.8.2623-2631.1997 (1997).

72 Holden, H. M., Meyer, T. E., Cusanovich, M. A., Daldal, F. \& Rayment, I. Crystallization and preliminary analysis of crystals of cytochrome $c_{2}$ from Rhodopseudomonas capsulata.J Mol Biol 195, 229-231, doi:10.1016/0022-2836(87)90341-x (1987).

73 Sheng, Q. et al. Preprocessing significantly improves the peptide/protein identification sensitivity of high-resolution isobarically labeled tandem mass spectrometry data. Mol Cell Proteomics 14, 405-417, doi:10.1074/mcp.O114.041376 (2015).

74 Pettersen, E. F. et al. UCSF Chimera--a visualization system for exploratory research and analysis. J Comput Chem 25, 1605-1612, doi:10.1002/jcc.20084 (2004).

75 Kosinski, J. et al. Xlink Analyzer: software for analysis and visualization of cross-linking data in the context of three-dimensional structures. J Struct Biol 189, 177-183, doi:10.1016/j.jsb.2015.01.014 (2015).

76 Zheng, S. et al. MotionCor2: anisotropic correction of beam-induced motion for improved cryoelectron microscopy. Nat Methods 14, 331-332, doi:10.1038/nmeth.4193 (2017).

77 Rohou, A. \& Grigorieff, N. CTFFIND4: fast and accurate defocus estimation from electron micrographs. J. Struct. Biol. 192, 216-221 (2015).

78 Zivanov, J. et al. New tools for automated high-resolution cryo-EM structure determination in RELION-3. eLife 7:e42166, doi:10.7554/eLife.42166 (2018).

79 Tang, G. et al. EMAN2: An extensible image processing suite for electron microscopy. J. Struct. Biol. 157, 38-46 (2007).

80 Liebschner, D. et al. Macromolecular structure determination using X-rays, neutrons and electrons: recent developments in Phenix. Acta Crystallogr D Struct Biol 75, 861-877, doi:10.1107/S2059798319011471 (2019).

81 Williams, C. J. et al. MolProbity: More and better reference data for improved all-atom structure validation. Protein Sci 27, 293-315, doi:10.1002/pro.3330 (2018).

82 Emsley, P., Lohkamp, B., Scott, W. G. \& Cowtan, K. Features and development of Coot. Acta Crystallogr D Biol Crystallogr 66, 486-501, doi:10.1107/S0907444910007493 (2010).

83 Webb, B. \& Sali, A. Comparative Protein Structure Modeling Using MODELLER. Curr Protoc Bioinformatics 47, 56 1-32, doi:10.1002/0471250953.bi0506s47 (2014).

84 Buchan, D. W. A. \& Jones, D. T. The PSIPRED Protein Analysis Workbench: 20 years on. Nucleic Acids Res 47, W402-W407, doi:10.1093/nar/gkz297 (2019).

85 Jones, D. T., Tress, M., Bryson, K. \& Hadley, C. Successful recognition of protein folds using threading methods biased by sequence similarity and predicted secondary structure. Proteins Suppl 3, 104-111, doi:10.1002/(sici)1097-0134(1999)37:3+<104::aid-prot14>3.3 .co;2-g (1999).

86 Chen, V. B. et al. MolProbity: all-atom structure validation for macromolecular crystallography. Acta Crystallogr D Biol Crystallogr 66, 12-21, doi:10.1107/S0907444909042073 (2010).

87 Yang, J. et al. The I-TASSER Suite: protein structure and function prediction. Nat Methods 12, 7-8, doi:10.1038/nmeth.3213 (2015).

88 Soding, J. Protein homology detection by HMM-HMM comparison. Bioinformatics 21, 951-960, doi:10.1093/bioinformatics/bti125 (2005).

89 Zhang, W. et al. Integration of QUARK and I-TASSER for Ab Initio Protein Structure Prediction in CASP11. Proteins 84 Suppl 1, 76-86, doi:10.1002/prot.24930 (2016). 
112690 Ko, J., Park, H., Heo, L. \& Seok, C. GalaxyWEB server for protein structure prediction and refinement. Nucleic Acids Res 40, W294-297, doi:10.1093/nar/gks493 (2012).

112891 Dong, G. Q., Fan, H., Schneidman-Duhovny, D., Webb, B. \& Sali, A. Optimized atomic statistical

1129 potentials: assessment of protein interfaces and loops. Bioinformatics 29, 3158-3166, doi:10.1093/bioinformatics/btt560 (2013). 
A e- transfer from CIII to CIV

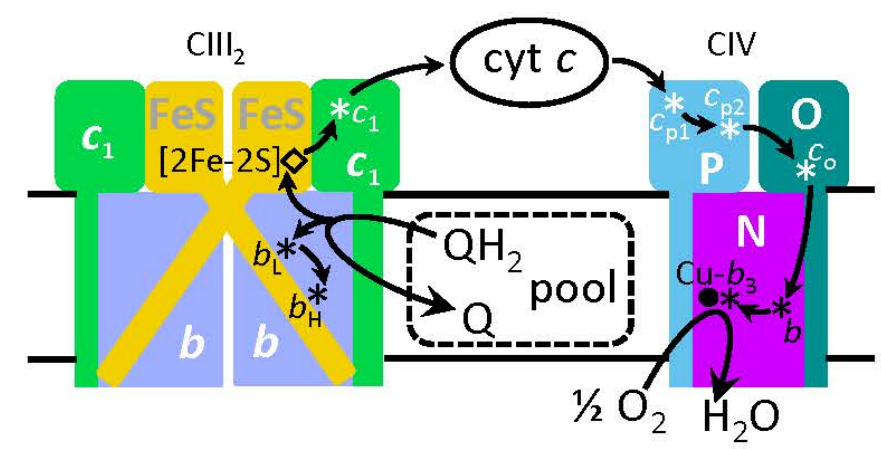

C Gene fusions on plasmid

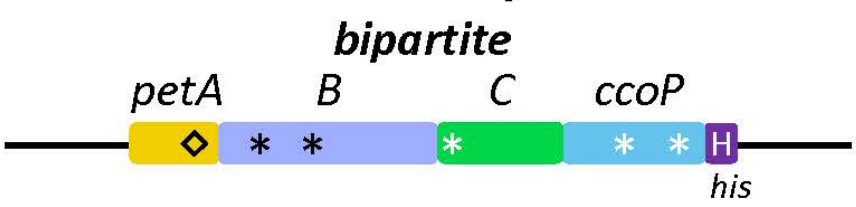

D CIII-CIV super-complexes (SC)

Bipartite SC

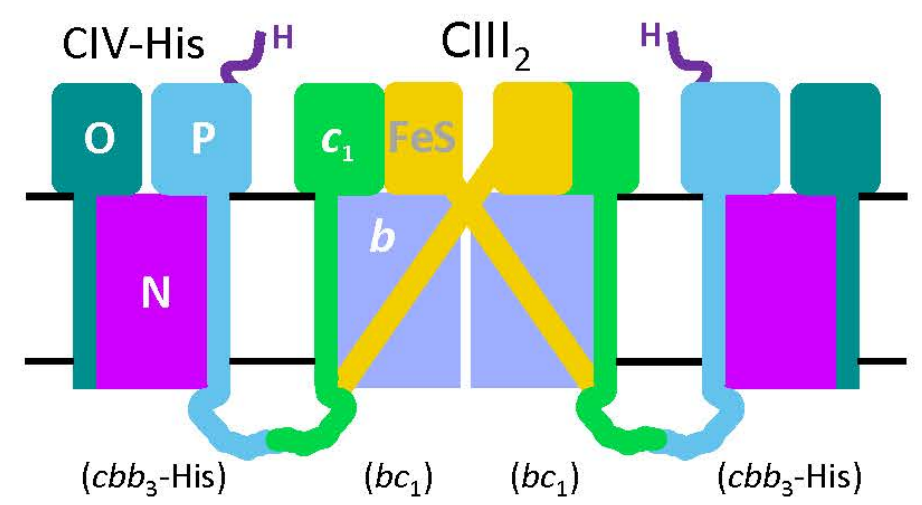

B Genes on chromosome

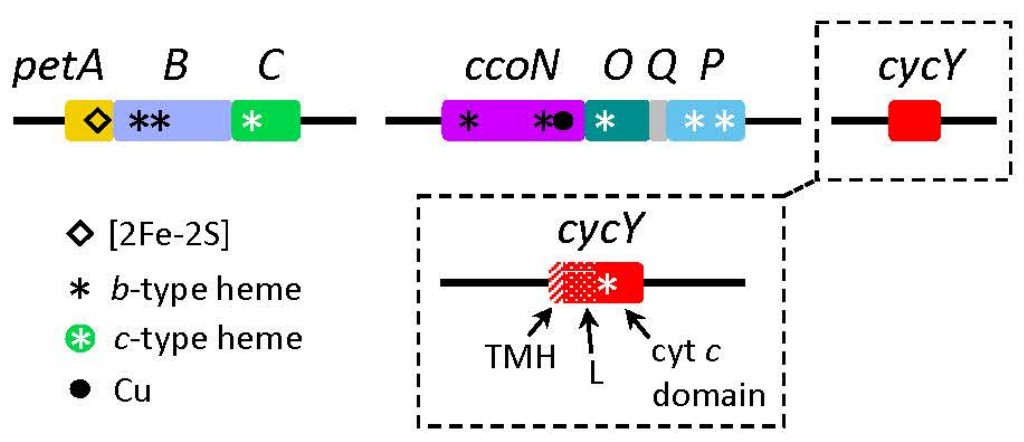


1133 Figure 1. $\mathrm{QH}_{2}$ oxidation and fused SCs. A. Oxidation of $\mathrm{QH}_{2}$ to $\mathrm{Q}$ by $\mathrm{CIII}_{2}$ and reduction of $\mathrm{O}_{2}$ to $\mathrm{H}_{2} \mathrm{O}$ by CIV. A bifurcated electron 1134 transfer reaction conveys one electron from $\mathrm{QH}_{2}$ to the $[2 \mathrm{Fe}-2 \mathrm{~S}]$ cluster of the FeS protein (FeS, yellow), and another electron to hemes $b_{\mathrm{L}}$ 1135 and $b_{\mathrm{H}}$ of cyt $b$ (periwinkle). Reduced [2Fe-2S] cluster transfers the electron to heme $c_{1}$ on cyt $c_{1}$ (green), via the movement of the FeS 1136 protein from near heme $b_{\mathrm{L}}$ to near heme $c_{1}$. The subsequent electron transfer step from heme $b_{\mathrm{H}}$ to $\mathrm{Q}$ from the pool to form a SQ 1137 (semiquinone) is not shown for clarity. A cyt $c\left(c_{2}\right.$ or $\left.c_{\mathrm{y}}\right)$ carries electrons from heme $c_{1}$ to $\mathrm{CIV}$, where electrons reach the heme-Cu (Cu$\left.1138 b_{3}\right)$ site, where $\mathrm{O}_{2}$ is reduced to $\mathrm{H}_{2} \mathrm{O}$, via the hemes $c_{\mathrm{p} 1}$ and $c_{\mathrm{p} 2}$ of $\mathrm{CcoP}(\mathrm{P}$, light blue $), c_{\mathrm{o}}$ of CcoO $(\mathrm{O}$, dark green $)$ and heme $b$ of CcoN $(\mathrm{N}$, 1139 purple). B. R. capsulatus genes used for fused SCs. pet $A B C$ encodes the structural genes of the $b c_{1}$-type $\mathrm{CIII}_{2}$ subunits, the FeS protein 1140 (petA, yellow), cyt $b\left(B\right.$, periwinkle) and cyt $c_{1}\left(C\right.$, green). $c c o N O Q P$ encodes the structural genes of the $c b b_{3}$-type CIV subunits, the CcoN 1141 ( $c c o N$, purple), $\mathrm{CcoO}\left(O\right.$, dark green), $\mathrm{CcoQ}\left(Q\right.$, grey) and $\mathrm{CcoP}\left(P\right.$, light blue). $c y c Y$ gene (red) encodes the membrane-anchored cyt $c_{\mathrm{y}}$, 1142 and its 30-residue transmembrane helix (TMH), 69-residue linker (L) and 100-residue cyt $c$ (cyt $c$ ) domain are indicated. Heme cofactors 1143 of $b$ - and $c$-type cyts are indicated by black and white asterisks, respectively, whereas diamond and dot designate the [2Fe-2S] cluster and $1144 \mathrm{Cu}$ atom, respectively. C. Plasmid-borne genetic fusions. The bipartite fusion (left) is formed by in-frame linking the 3'-end of petC to the 11455 '-end of $c c o P$, and the tripartite fusion (right) by adding the linker and cyt $c$ domain of $c y c Y$ to the 3'-end of $c c o P$. Colors and cofactor 1146 symbols are as in $\mathbf{A}$, and the His (H) and Flag (F) affinity tags (dark purple) at the 3'-end of the bipartite and tripartite fusion subunits are 1147 shown. D. Schematic depiction of bipartite (left) and tripartite (right) super-complexes (SC). The bipartite $\mathrm{SC}_{\text {encodes a CIII }}$ dimer fused 1148 on each side to a His-tagged CIV. The tripartite SC also contains the Flag-tagged cyt domain of $c_{\mathrm{y}}$ (red) at the end of CcoP (blue). 


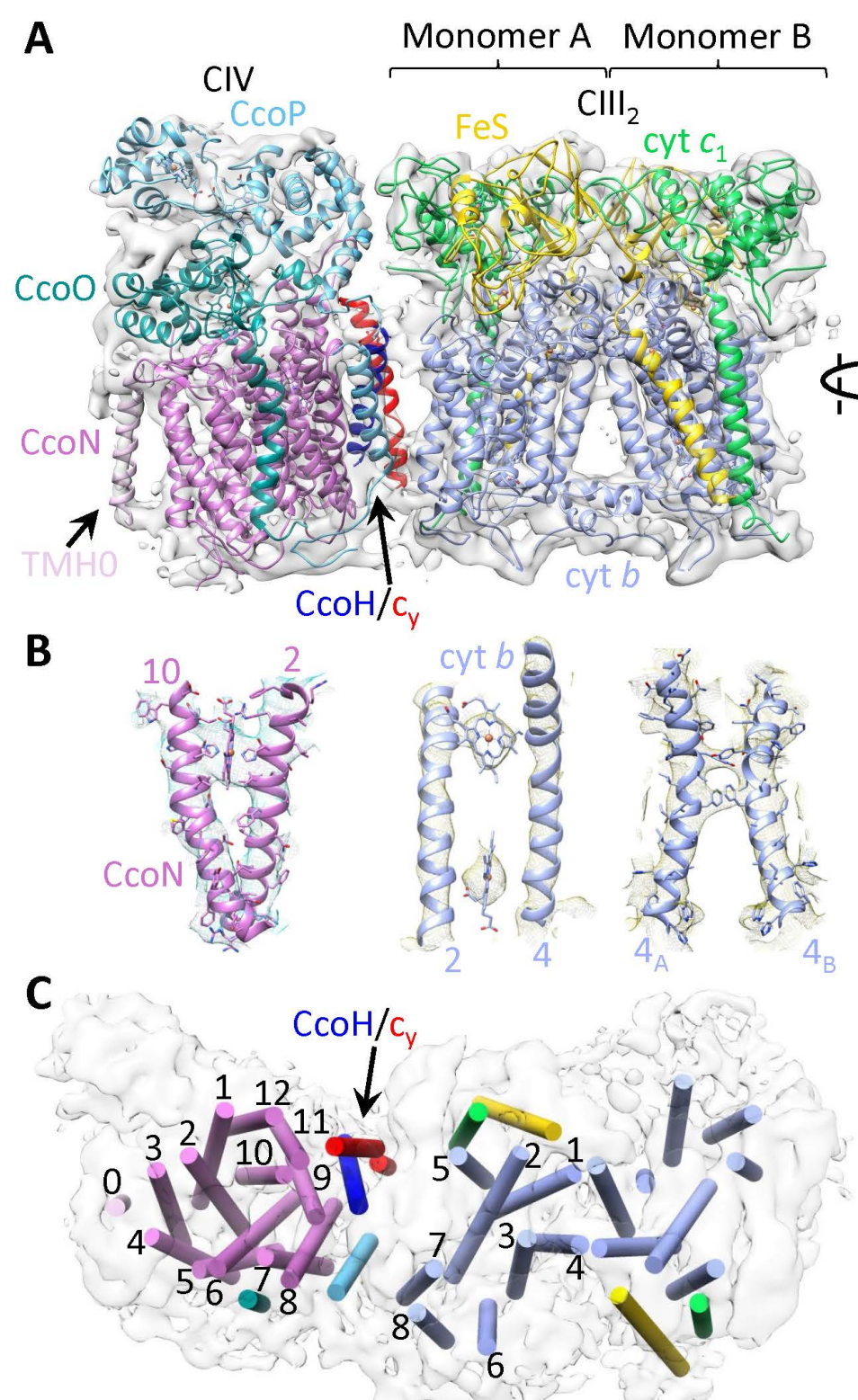

1150
D $\mathrm{ClII}_{2} \quad \mathrm{CIV}$
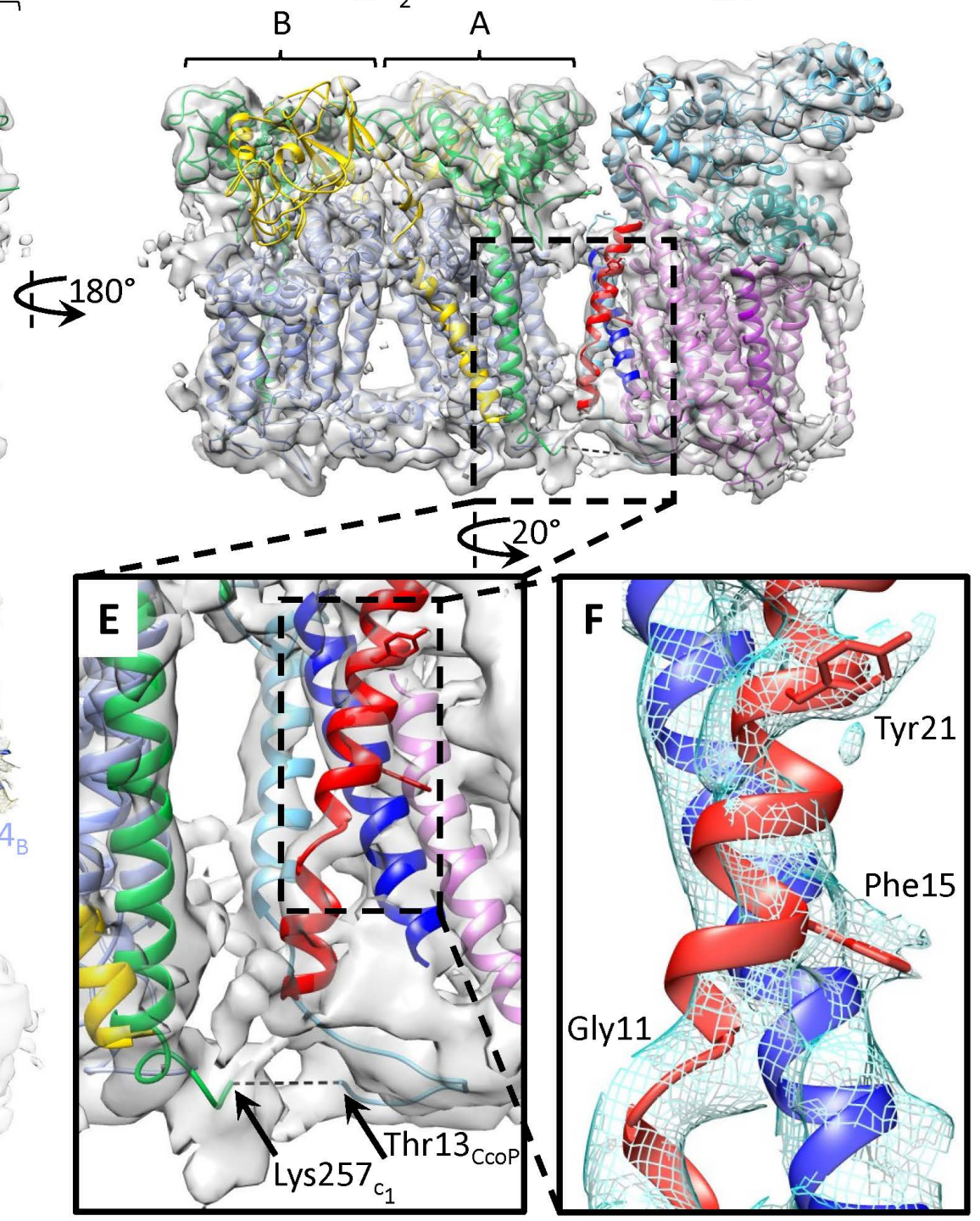

Figure 2. Steimle et al., 
1151 Figure 2. Cryo-EM structure of $\mathrm{CIII}_{2} \mathrm{CIV}$. A. Side view of $\mathrm{CIII}_{2} \mathrm{CIV}$. The structures of CIII 2 (PDB: 6XI0), and the homology model of $1152 R$. capsulatus $c b b_{3}$-type CIV were fitted into the cryo-EM map SC-2A depicted in transparent grey. All subunits are colored and labelled 1153 as indicated, and the additional feature indicated by an arrow at the edge of CcoN subunit of CIV corresponds to the extra N-ter TMH 1154 (TMH0, light purple) specific to $R$. capsulatus. The large arrow points out the $\mathrm{CcoH} / c_{\mathrm{y}}$ helices in red/blue. B. Representative regions of 1155 the cryo-EM map showing the map quality and model fitting. The TMH2 and TMH10 of CcoN (left) show clearly heme $b$ and some bulky 1156 side chains. Only the protein backbone and hemes $b_{\mathrm{L}}$ and $b_{\mathrm{H}}$ are resolved between the TMH2 and TMH4 of cyt $b$ (center) (see Fig. 3B for 1157 comparison with $\mathrm{CIII}_{2}$ map at $3.3 \AA$ A). Large side chains are clearly visible between the TMH4 of monomer A and TMH4 of monomer B of $1158 \mathrm{CIII}_{2}\left(4_{\mathrm{A}}\right.$ and $4_{\mathrm{B}}$, respectively; right). C. Top view of CIII ${ }_{2} \mathrm{CIV}$ with TMHs depicted as cylinders and colored as in A. The TMHs of cyt $b$ 1159 (only $\mathrm{CIII}_{2}$ monomer A) and $\mathrm{CcoN}$ of CIV are numbered, and the TMHs of the FeS protein (yellow), cyt $c_{1}$ (green), CcoO (dark green), $1160 \mathrm{CcoP}$ (light blue), $\mathrm{CcoH} / c_{\mathrm{y}}$ (blue/red with an arrow) and CcoN TMH0 (light purple) are shown. D. $180^{\circ}$ rotated view for the back view of $1161 \mathrm{CIII}_{2} \mathrm{CIV}$ interface. The two extra TMHs at the interface are attributed to those of CcoH (blue) and cyt $c_{\mathrm{y}}$ (red). An additional TMH at the 1162 edge of CIV is attributed to the predicted N-ter TMH of CcoN (TMH0, light purple), and depicted as an ab initio model (Methods). E. 1163 Enlarged view of $\mathrm{CIII}_{2} \mathrm{CIV}$ interface. The view is slightly rotated relative to $\mathbf{A}$ for better visibility of CcoP TMH in the background (light 1164 blue). Only CcoN TMH9 is shown next to CcoH (blue) and cyt $c_{\mathrm{y}}$ (red) TMHs. The fusion region between cyt $c_{1}$ and CcoP is indicated at 1165 the bottom, with the C-ter of cyt $c_{1}$ (green) and the N-ter (resolved portion in the map) of CcoP (light blue) and their respective terminal 1166 residues (Lys257 1 and $\left.\mathrm{Thr} 13_{\mathrm{CcoP}}\right)$. The $12 \mathrm{~N}$-ter CcoP residues connecting these two chains (dashed line) are not clearly resolved. F. 1167 Enlarged view showing close interaction between the $\mathrm{CcoH}$ and cyt $c_{\mathrm{y}} \mathrm{TMHs}$. Characteristic features of cyt $c_{\mathrm{y}} \mathrm{TMH}\left(\mathrm{NH}_{2}-\right.$ 
1168 Gly11xxxPhe15xxxx Tyr21-COOH) are used to determine the registration. The helix break induced by Gly11, and the bulky sidechains 1169 for Phe15 and Tyr21 are clearly visible.

1170

1171 
A $\mathrm{ClII}_{2}$

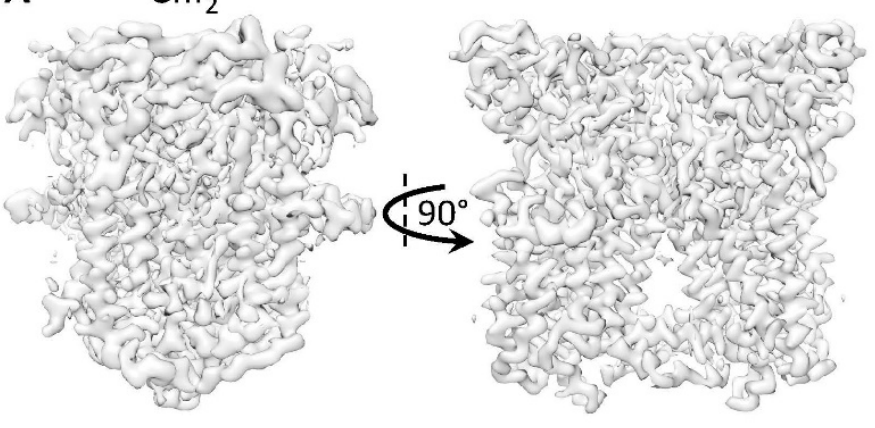

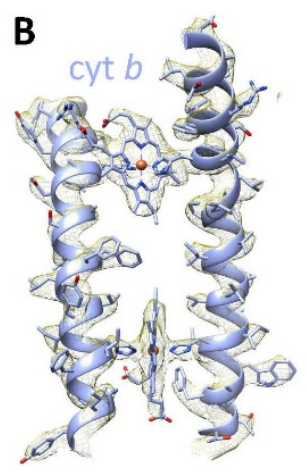

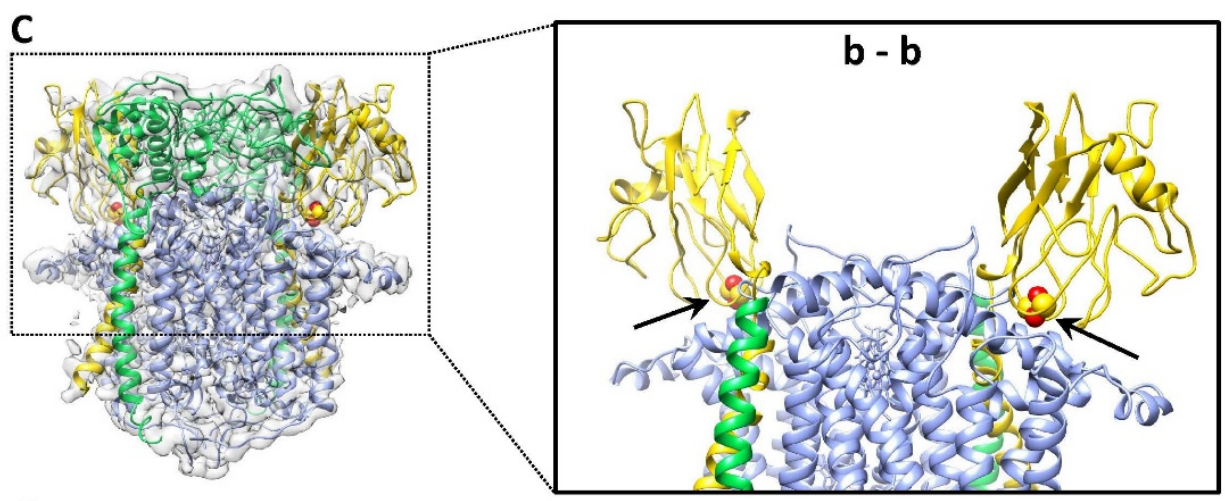

D
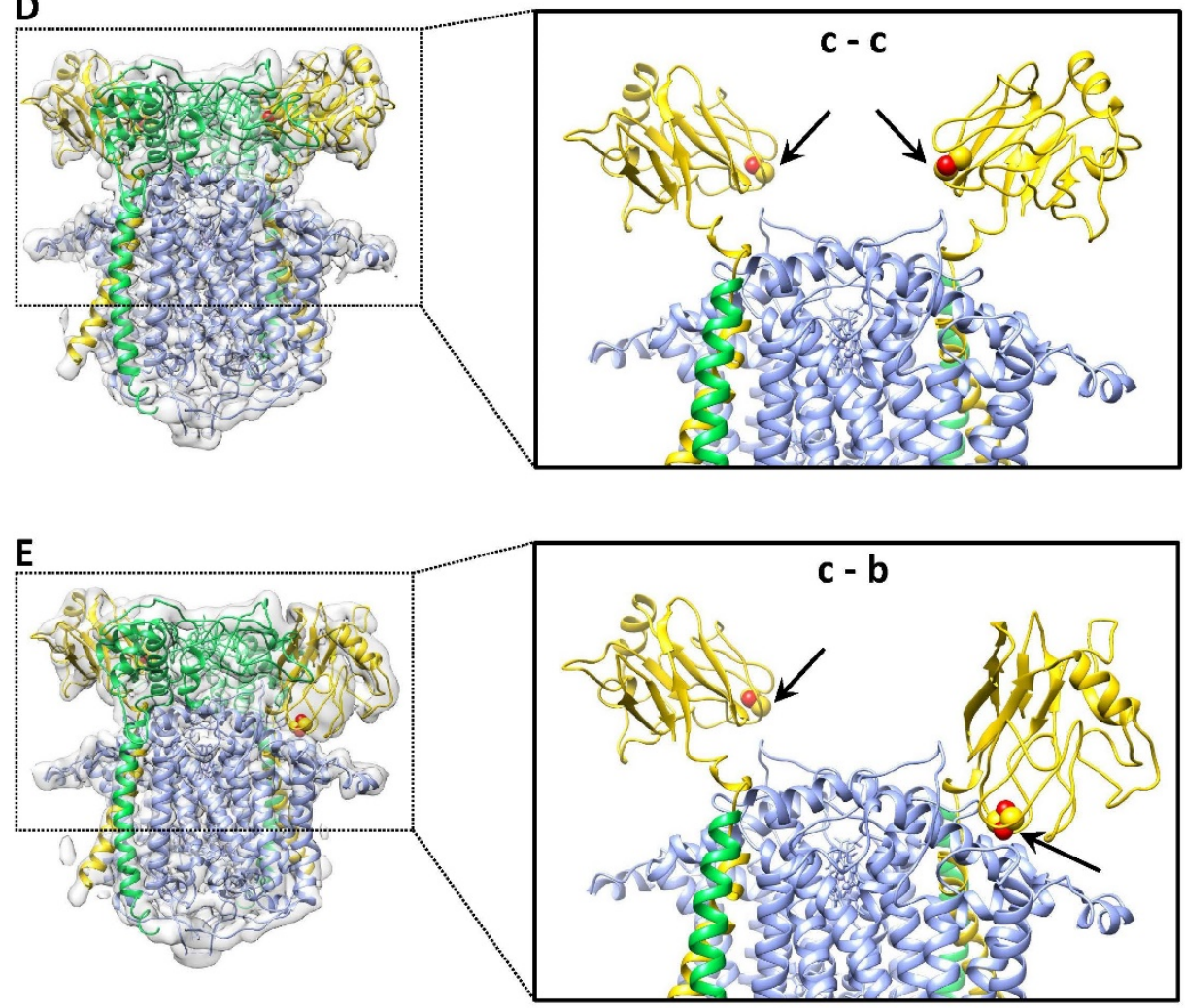

Figure 3. Steimle et al., 
1173 Figure 3: Structures of native CIII $_{2}$ conformers. A. Cryo-EM map CIII 2 b-b (EMD-22226) with

1174 both FeS proteins in b position. B. Representative region of $\mathrm{CIII}_{2}$ b-b demonstrating map quality and 1175 model fitting. TMH2 and TMH4 of cyt $b$ with hemes $b_{\mathrm{L}}$ and $b_{\mathrm{H}}$ are shown. C-E. Maps and models 1176 showing different conformations of the FeS proteins. The left panels show the $\mathrm{CIII}_{2}$ structure fitted 1177 into the different maps with the subunit colorings (cyt $b$ in periwinkle, cyt $c_{1}$ in green, and the FeS 1178 protein in yellow) as in Fig. 2. The right panels show the top half of the models with the membrane1179 external domain of cyt $c_{1}$ omitted to better visualize the $\mathrm{b}-\mathrm{b}, \mathrm{c}-\mathrm{c}$ and $\mathrm{c}-\mathrm{b}$ positions of FeS-EDs.

1180 The $[2 \mathrm{Fe}-2 \mathrm{~S}]$ clusters are shown as yellow-red spheres and indicated by arrows. C. Structure of native $1181 \mathrm{CIII}_{2}$ with both FeS-EDs in b position (map $\mathrm{CII}_{2}$ b-b, EMD-22226; PDB: 6XKV). D. Structure of 1182 native $\mathrm{CIII}_{2}$ with both FeS-EDs in c position (map $\mathrm{CIII}_{2}$ c-c, EMD-22224; PDB: 6XKT). and E. 1183 Structure of native $\mathrm{CIII}_{2}$ with one FeS-ED in c and one in b position (map CIII ${ }_{2}$ b-c, EMD-22225; 1184 PDB: 6XKU). 

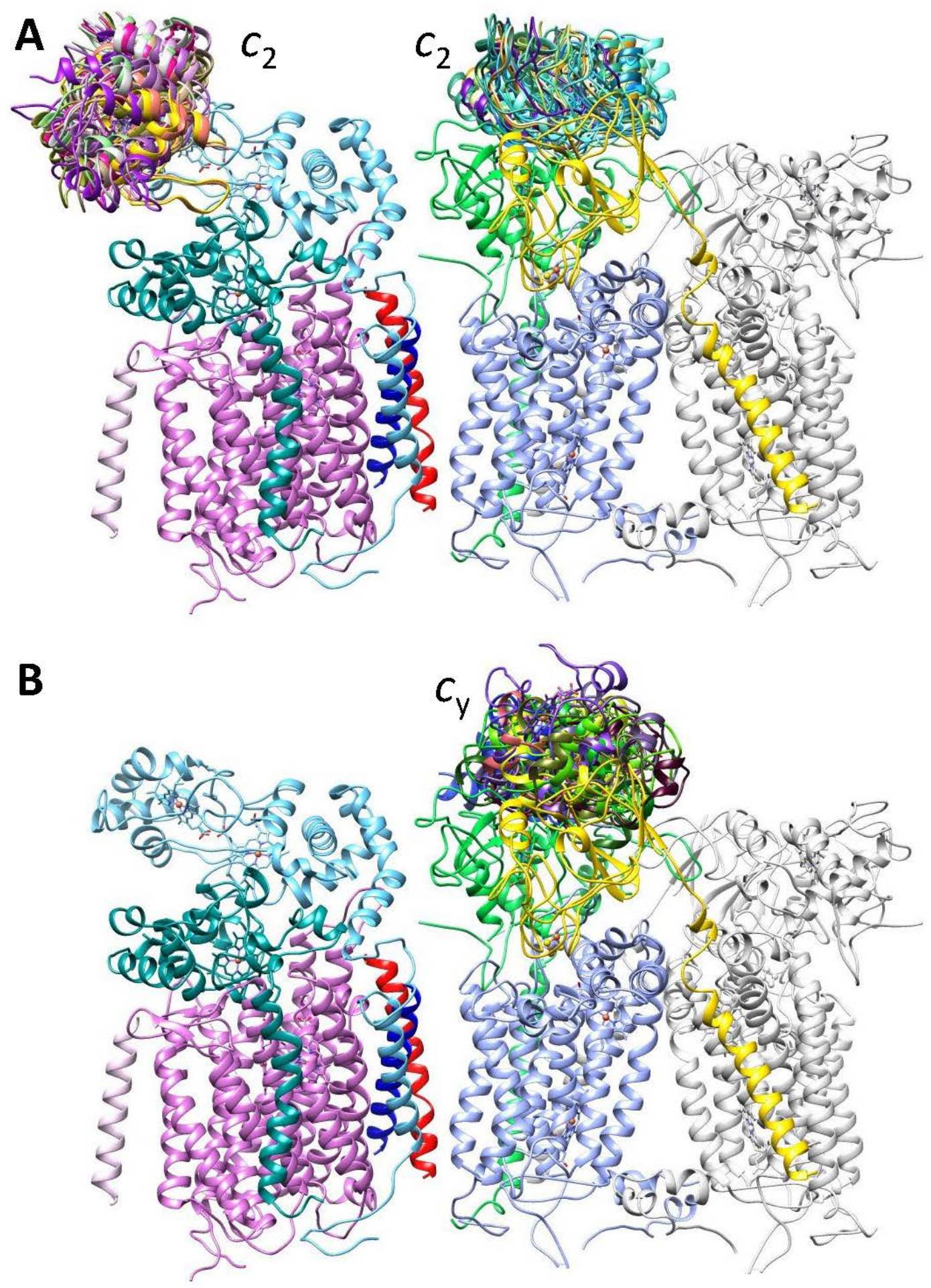

Figure 4. Steimle et al, 
1186 Figure 4: Binding regions of cyt $c_{2}$ and cyt $c_{\mathbf{y}}$ on $\mathbf{C I I I}_{2} \mathbf{C I V}$. The binding regions were defined by

1187 XL-MS guided docking, and the subunits of $\mathrm{CIII}_{2} \mathrm{CIV}$ are colored as in Fig. 2 except the monomer

$1188 \mathrm{~B}$ of $\mathrm{CIII}_{2}$ shown in light grey. Only the binding regions on monomer A are shown. A. Cyt $c_{2}$ (PDB:

1189 1C2N) was docked onto $\mathrm{CIII}_{2}$ and CIV using Patchdock guided by DMTMM generated XLs as

1190 distance restraints. This yielded one cluster of models on CIV and one per monomer of CIII 2 . B. A

1191 model of cyt domain of $c_{\mathrm{y}}$, generated using $P$. denitrificans cyt $c_{552}$ structure (PDB: 3M97) as a

1192 template (RMSD between template and model: $0.2 \AA$ ) was docked on $\mathrm{CIII}_{2}$ as in $\mathbf{A}$, except that both

1193 DMTMM and DSBU generated XLs provided distance restraints. Two binding clusters for cyt

1194 domain of $c_{\mathrm{y}}$ per monomer of $\mathrm{CIII}_{2}$ were found. The two clusters are located behind each other on

1195 a side view, but are clearly visible on top views (Fig. S4F-H, labeled 1 and 2). Only cluster 1 which

1196 is closer to cyt $c_{1}$ and overlapping with the binding region of cyt $c_{2}$ is shown. In all cases, 10

1197 representative models are shown to visualize the clusters of binding models. No binding region for

1198 cyt $c_{\mathrm{y}}$ on CIV could be defined since no XL was found between the proteins.

1199 


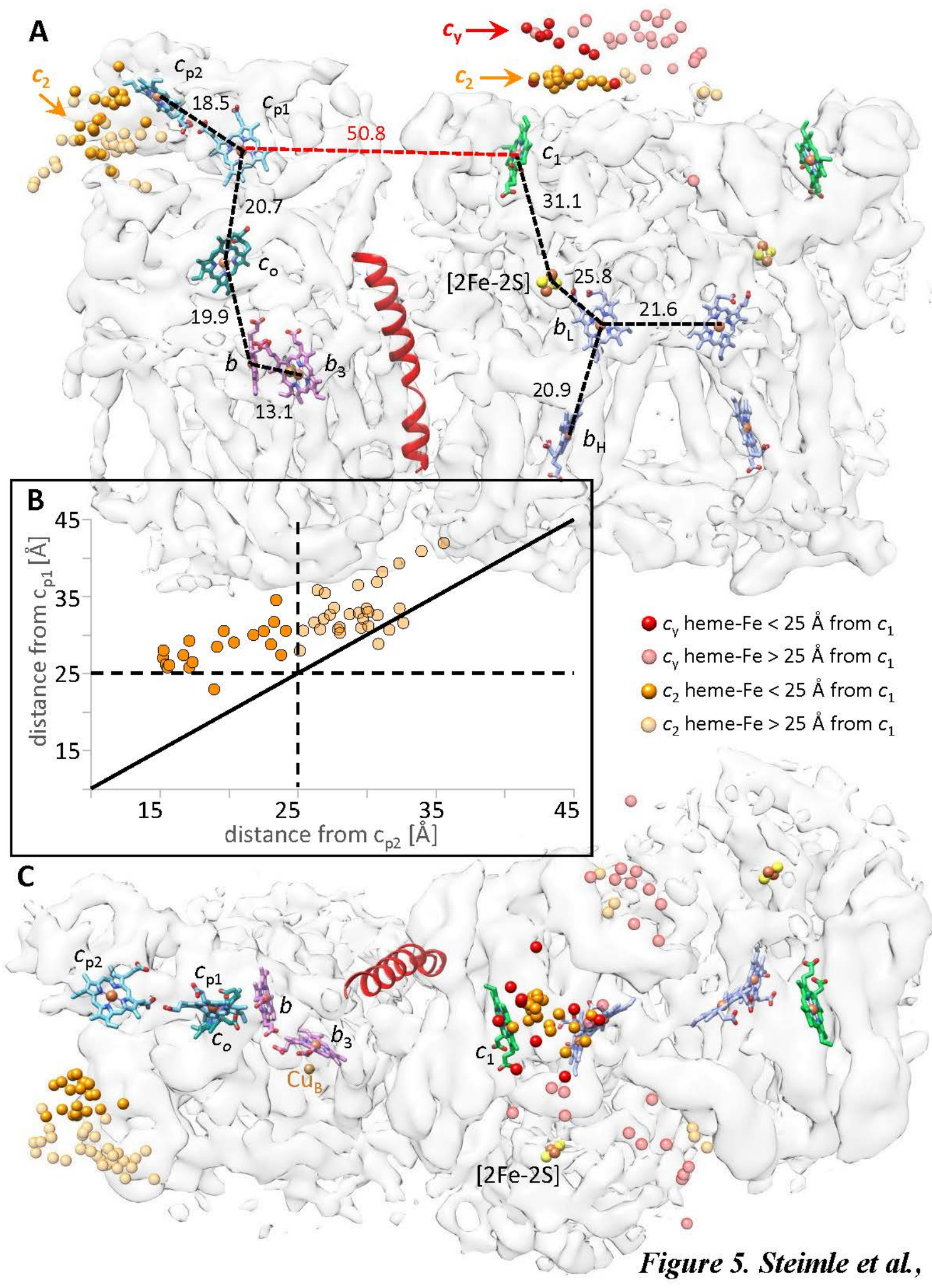


1201 Figure 5. Organization of $\mathbf{C I I I}_{2} \mathbf{C I V}$ cofactors and redox partners. A. The cofactors are shown

1202 inside the transparent map SC-2A of $\mathrm{CIII}_{2} \mathrm{CIV}$ with the same subunit colors as in Fig. 2: hemes $b_{\mathrm{L}}$

1203 and $b_{\mathrm{H}}$ (periwinkle), heme $c_{1}$ (green), hemes $c_{\mathrm{p} 1}$ and $c_{\mathrm{p} 2}$ (light blue), heme $c_{\mathrm{o}}$ (dark green), hemes $b$

1204 and $b_{3}$ (purple). The [2Fe-2S] clusters are shown as yellow-red spheres. In all cases the distances

1205 (heme-Fe to heme-Fe) between the heme cofactors are indicated. The positions of docked cyt $c_{2}$ and

1206 cyt domain of $c_{\mathrm{y}}$ are indicated as orange (heme $c_{2}$ ) and red (heme $c_{\mathrm{y}}$ ) spheres, respectively,

1207 representing their heme-Fe atoms. All heme-Fe atoms corresponding to the top 50 docking positions

1208 for cyt $c_{2}$ on CIV are shown as solid $(<25 \AA)$ or transparent $(>25 \AA)$ spheres, depending on their

1209 distances to heme $c_{\mathrm{p} 2}$. In the case of $\mathrm{CIII}_{2}$, only the docking positions of cyt $c_{2}$ and cyt $c_{\mathrm{y}}$ on monomer

1210 A and between the monomers A and B are shown, omitting those located on monomer B. The TMH

1211 of cyt $c_{\mathrm{y}}$ is shown in red at $\mathrm{CIII}_{2} \mathrm{CIV}$ interface. $\mathbf{B}$. The heme-Fe atoms of all 50 cyt $c_{2}$ models docked

1212 onto CIV are plotted in function of their distances from heme $c_{\mathrm{p} 1}$ and heme $c_{\mathrm{p} 2}$, with the $\mathrm{Fe}$ atoms

1213 within $25 \AA ̊$ shown as solid spheres, and those beyond $25 \AA$ as transparent spheres. The vast majority

1214 of heme-Fe atoms of docked cyt $c_{2}$ models are closer to heme $c_{\mathrm{p} 2}$ than heme $c_{\mathrm{p} 1}$ of CIV. C. Top view

1215 of the map shown in $\mathbf{A}$ is presented to better visualize the distribution of the docked cyt domain of

$1216 c_{\mathrm{y}}$ on monomer A and between the monomers A and B. In all cases, the heme-Fe atoms are depicted

1217 by spheres and colored as indicated above and on the figure.

1218 


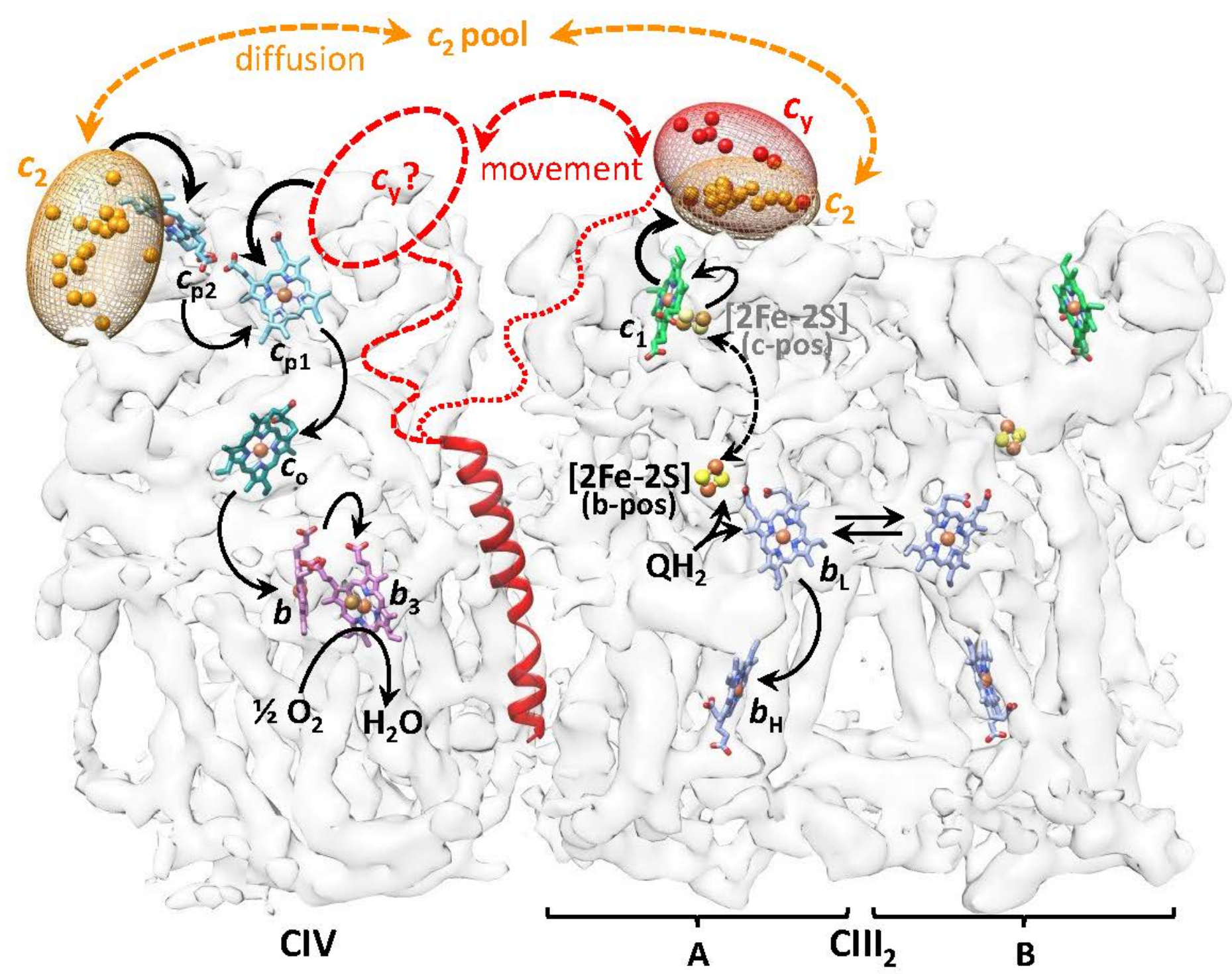

Figure 6. Steimle et al., 

and cyt $c$ domains of $c_{\mathrm{y}}$ (orange and red ellipsoids, respectively), defined by XL-MS guided docking, are depicted by the distributions of their heme-Fe atoms on the transparent map SC-2A of CIII ${ }_{2} \mathrm{CIV}$. Only the positions that are within $25 \AA$ of heme $c_{1}$ of CIII ${ }_{2}$ or heme $c_{\mathrm{p} 2}$ of CIV are indicated. The cofactors together with the TMH of cyt $c_{\mathrm{y}}$ are shown as in Fig. 5. The linker region (indicated by dotted or dashed lines) between the TMH and the cyt domain of $c_{\mathrm{y}}$ is not resolved in the cryo-EM map. The proposed electron transport pathways are shown by thicker black arrows: upon $\mathrm{QH}_{2}$ oxidation by $\mathrm{CIII}_{2}$, cyt $c_{\mathrm{y}}$ which is integral to $\mathrm{CIII}_{2} \mathrm{CIV}$ receives an electron from heme $c_{1}$. It then moves (double-headed dashed red arrow) to an undefined binding region (dashed oval with $c_{\mathrm{y}}$ ?) on CIV, where it delivers the electron to the nearest heme $c_{\mathrm{p} 1}$ of CIV. Similarly, cyt $c_{2}$ which is peripheral to $\mathrm{CIII}_{2} \mathrm{CIV}$ also receives an electron from heme $c_{1}$, diffuses away to reach are indicated by thinner arrows. The double headed dashed black arrow depicts the movement of the $[2 \mathrm{Fe}-2 \mathrm{~S}]$ of FeS protein from the $b$ position (b-pos, black, solid spheres) to the c position (c-pos, grey, transparent spheres) in $\mathrm{CIII}_{2}$ during $\mathrm{QH}_{2}$ oxidation. A possible electron equilibration between the two heme $b_{\mathrm{L}}$ of $\mathrm{CIII}_{2}$ is indicated by double arrows, and the electron transfer steps subsequent to heme $b_{\mathrm{H}}$ reduction are not shown. 


\section{Figures}

A e- transfer from CIII to CIV

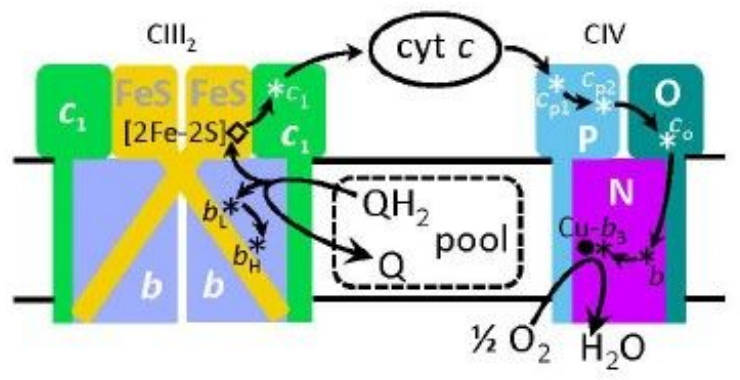

C Gene fusions on plasmid bipartite

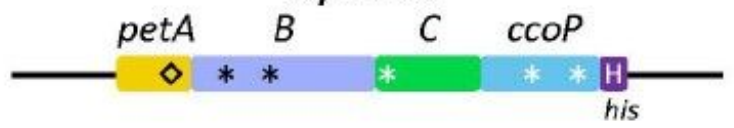

D CIII-CIV super-complexes (SC) Bipartite SC

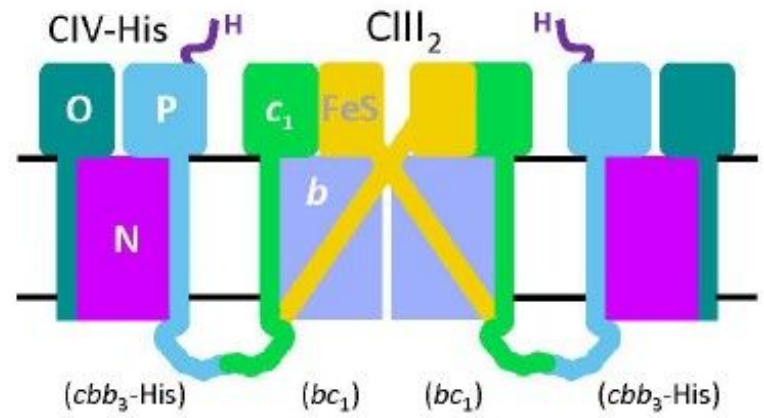

B Genes on chromosome
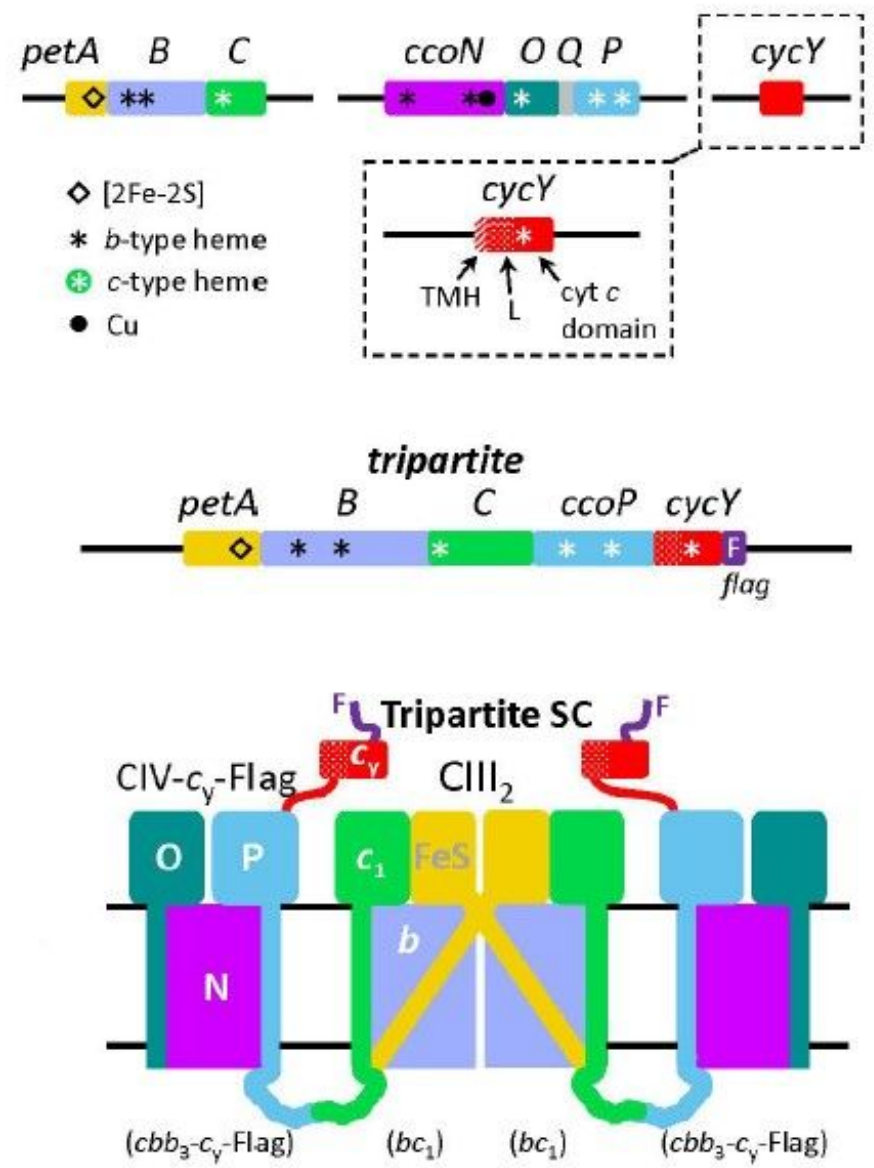

\section{Figure 1}

$\mathrm{QH} 2$ oxidation and fused SCs. A. Oxidation of $\mathrm{QH} 2$ to $\mathrm{Q}$ by $\mathrm{Clll} 2$ and reduction of $\mathrm{O} 2$ to $\mathrm{H} 2 \mathrm{O}$ by $\mathrm{CIV}$. 1133 A bifurcated electron transfer reaction conveys one electron from $\mathrm{QH} 2$ to the [2Fe-2S] cluster of the FeS protein (FeS, yellow), and another electron to hemes bL and bH of cyt b (periwinkle). Reduced [2Fe-2S] cluster transfers the electron to heme $\mathrm{c} 1$ on cyt $\mathrm{c} 1$ (green), via the movement of the FeS protein from near heme $b L$ to near heme $c 1$. The subsequent electron transfer step from heme $b H$ to $Q$ from the pool to form a SQ (semiquinone) is not shown for clarity. A cyt c (c2 or cy) carries electrons from heme c1 to CIV, where electrons reach the heme- $\mathrm{Cu}(\mathrm{Cu}$ b3) site, where $\mathrm{O} 2$ is reduced to $\mathrm{H} 2 \mathrm{O}$, via the hemes $\mathrm{cp} 1$ and cp2 of $\mathrm{CcoP}$ (P, light blue), $\mathrm{co}$ of $\mathrm{CcoO}(\mathrm{O}$, dark green) and heme b of $\mathrm{CcoN}(\mathrm{N}$, purple). B. R. capsulatus genes used for fused SCs. petABC encodes the structural genes of the bc1-type CIII2 subunits, the FeS protein (petA, yellow), cyt b (B, periwinkle) and cyt $\mathrm{c} 1$ ( $C$, green). ccoNOQP encodes the structural genes of the cbb3-type CIV subunits, the $\mathrm{CcoN}$ (ccoN, purple), $\mathrm{CcoO}(\mathrm{O}$, dark green), $\mathrm{CcoQ}(\mathrm{Q}$, grey) and $\mathrm{CcoP}$ (P, light blue). cycY gene (red) encodes the membrane-anchored cyt cy, and its 30-residue transmembrane helix $(\mathrm{TMH}), 69$-residue linker $(\mathrm{L})$ and 100-residue cyt c (cyt $\mathrm{c}$ ) domain are indicated. Heme cofactors of b-and 
c-type cyts are indicated by black and white asterisks, respectively, whereas diamond and dot designate the [2Fe-2S] cluster and $\mathrm{Cu}$ atom, respectively. C. Plasmid-borne genetic fusions. The bipartite fusion (left) is formed by in-frame linking the 3 '-end of petC to the $5^{\prime}$-end of ccoP, and the tripartite fusion (right) by adding the linker and cyt $\mathrm{c}$ domain of cycY to the $3^{\prime}$-end of ccoP. Colors and cofactor symbols are as in A, and the His $(\mathrm{H})$ and Flag $(\mathrm{F})$ affinity tags (dark purple) at the 3 '-end of the bipartite and tripartite fusion subunits are shown. D. Schematic depiction of bipartite (left) and tripartite (right) super-complexes (SC). The bipartite SC encodes a CIII2 dimer fused on each side to a His-tagged CIV. The tripartite SC also contains the Flag-tagged cyt domain of cy (red) at the end of CcoP (blue).

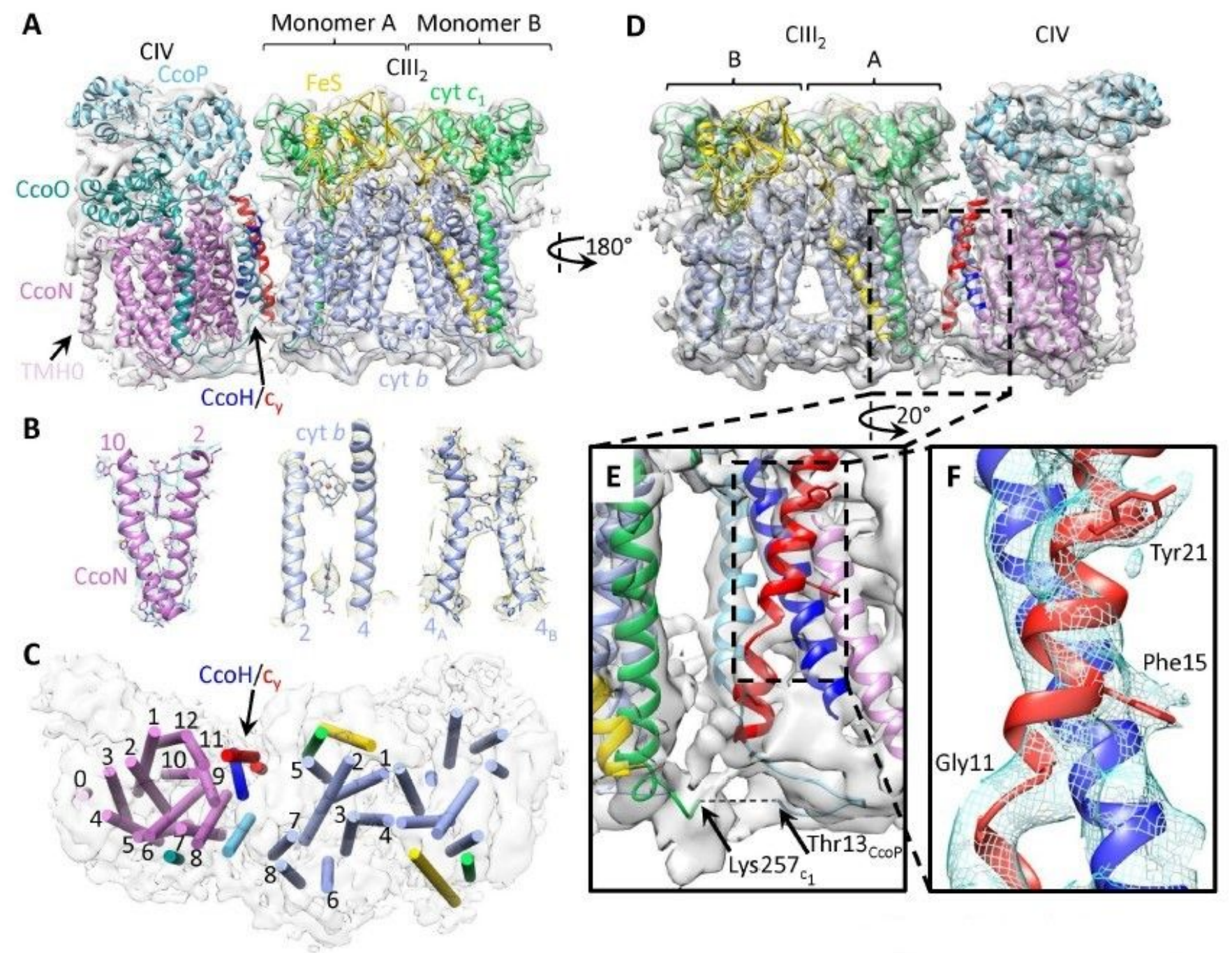

Figure 2

Cryo-EM structure of CIII2CIV. A. Side view of CIII2CIV. The structures of CIII2 (PDB: 6XI0), 1151 and the homology model of R. capsulatus cbb3-type CIV were fitted into the cryo-EM map SC-2A depicted in transparent grey. All subunits are colored and labelled as indicated, and the additional feature indicated by an arrow at the edge of $\mathrm{CcoN}$ subunit of CIV corresponds to the extra N-ter TMH (TMHO, light purple) specific to R. capsulatus. The large arrow points out the $\mathrm{CcoH} /$ cy helices in red/blue. B. Representative 
regions of the cryo-EM map showing the map quality and model fitting. The TMH2 and TMH10 of CcoN (left) show clearly heme $b$ and some bulky side chains. Only the protein backbone and hemes bL and bH are resolved between the TMH2 and TMH4 of cyt b (center) (see Fig. 3B for comparison with CIII2 map at $3.3 \AA$ ). Large side chains are clearly visible between the TMH4 of monomer A and TMH4 of monomer $\mathrm{B}$ of CIII2 (4A and 4B, respectively; right). C. Top view of CIII2CIV with TMHs depicted as cylinders and colored as in A. The TMHs of cyt b (only CIII2 monomer A) and CcoN of CIV are numbered, and the TMHs of the FeS protein (yellow), cyt c1 (green), $\mathrm{CcoO}$ (dark green), $\mathrm{CcoP}$ (light blue), $\mathrm{CcoH} / \mathrm{cy}$ (blue/red with an arrow) and CcoN TMHO (light purple) are shown. D. $180^{\circ}$ rotated view for the back view of CIII2CIV interface. The two extra TMHs at the interface are attributed to those of $\mathrm{CcoH}$ (blue) and cyt cy (red). An additional TMH at the edge of CIV is attributed to the predicted N-ter TMH of CcoN (TMHO, light purple), and depicted as an ab initio model (Methods). E. Enlarged view of CIII2CIV interface. The view is slightly rotated relative to $\mathrm{A}$ for better visibility of $\mathrm{CcOP}$ TMH in the background (light blue). Only CcoN TMH9 is shown next to $\mathrm{CcoH}$ (blue) and cyt cy (red) TMHs. The fusion region between cyt $\mathrm{c} 1$ and CcoP is indicated at the bottom, with the C-ter of cyt $\mathrm{c} 1$ (green) and the N-ter (resolved portion in the map) of CcoP (light blue) and their respective terminal residues (Lys257c1 and Thr13CcoP). The $12 \mathrm{~N}$-ter CcoP residues connecting these two chains (dashed line) are not clearly resolved. F. Enlarged view showing close interaction between the $\mathrm{CcoH}$ and cyt cy TMHs. Characteristic features of cyt cy TMH (NH2-51 Gly11xxxPhe15xxxxxTyr21- $\mathrm{COOH}$ ) are used to determine the registration. The helix break induced by Gly11, and the bulky sidechains for Phe15 and Tyr21 are clearly visible. 

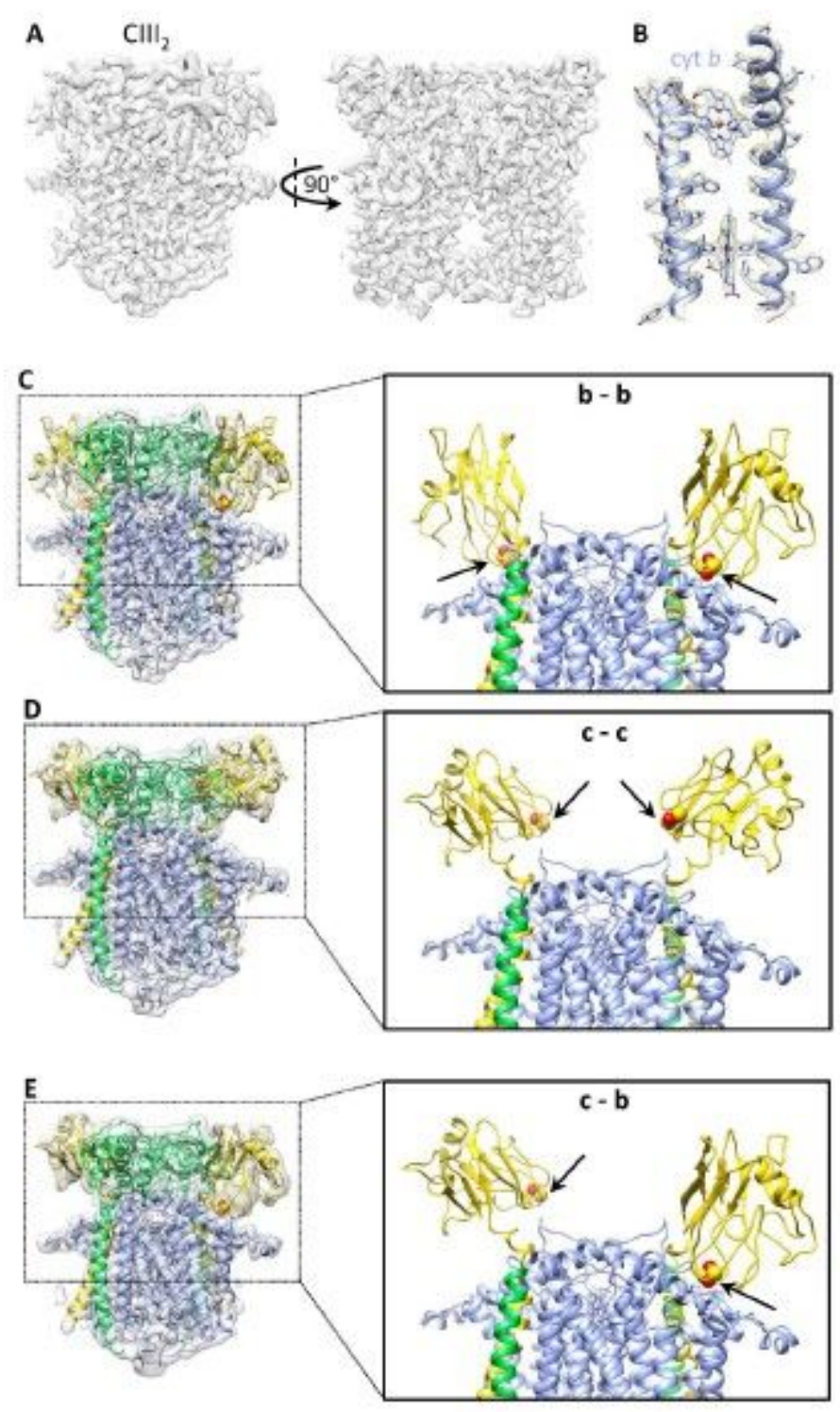

Figure 3

Structures of native CIII2 conformers. A. Cryo-EM map 1173 CIII2 b-b (EMD-22226) with both FeS proteins in $\mathrm{b}$ position. $\mathrm{B}$. Representative region of $\mathrm{ClII} 2 \mathrm{~b}-\mathrm{b}$ demonstrating map quality and model fitting. TMH2 and $\mathrm{TMH} 4$ of cyt $\mathrm{b}$ with hemes $\mathrm{bL}$ and $\mathrm{bH}$ are shown. C-E. Maps and models showing different conformations of the FeS proteins. The left panels show the CIII2 structure fitted into the different maps with the subunit colorings (cyt b in periwinkle, cyt c1 in green, and the FeS protein in yellow) as in Fig. 2. The right panels show the top half of the models with the membrane external domain of cyt $\mathrm{c} 1$ omitted to better visualize the $b-b, c-c$ and $c-b$ positions of FeS-EDs. The [2Fe-2S] clusters are shown as yellowred spheres and indicated by arrows. C. Structure of native CIII2 with both FeS-EDs in b position (map CIII2 b-b, EMD-22226; PDB: 6XKV). D. Structure of native CIII2 with both FeS-EDs in c position (map CIII2 C-c, EMD-22224; PDB: 6XKT). and E. Structure of native CIII2 with one FeS-ED in $c$ and one in b position (map CIII2 b-c, EMD-22225; PDB: 6XKU). 

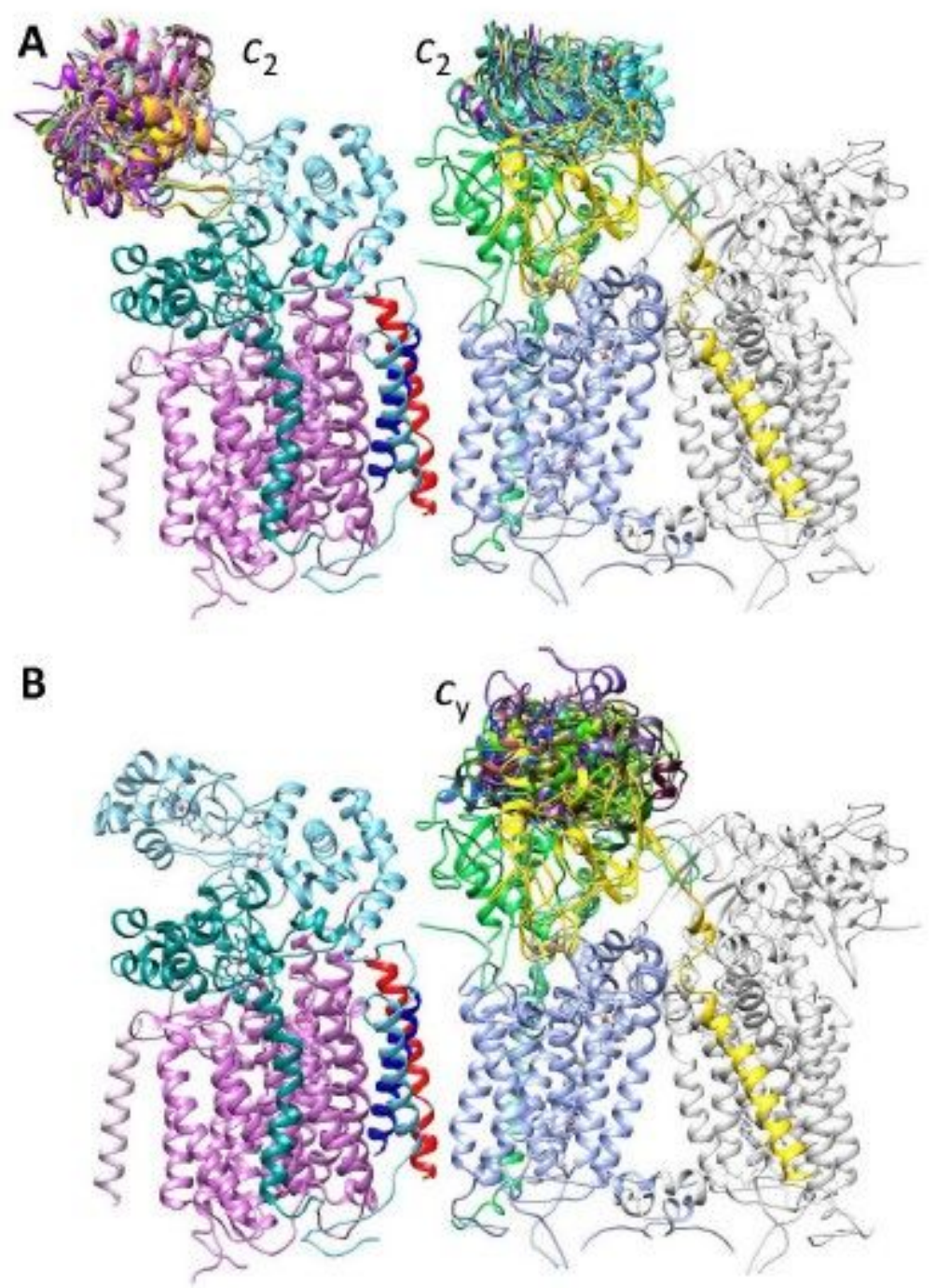

\section{Figure 4}

Binding regions of cyt $\mathrm{c} 2$ and cyt cy on CIII2CIV. The binding 1186 regions were defined by XL-MS guided docking, and the subunits of CIII2CIV are colored as in Fig. 2 except the monomer B of CIII2 shown in light grey. Only the binding regions on monomer A are shown. A. Cyt C2 (PDB: 1C2N) was docked onto CIII2 and CIV using Patchdock guided by DMTMM generated XLs as distance restraints. This yielded one cluster of models on CIV and one per monomer of CIII2. B. Amodel of cyt domain of cy, generated using P. denitrificans cyt c552 structure (PDB: 3M97) as a template (RMSD between template and model: $0.2 \AA$ ) was docked on CIII2 as in A, except that both DMTMM and DSBU generated XLs provided distance restraints. Two binding clusters for cytdomain of cy per monomer of CIII2 were found. The two clusters are located behind each other on a side view, but are clearly visible on top views (Fig. S4F-H, labeled 1 and 2). Only cluster 1 which is closer to cyt $\mathrm{c} 1$ and overlapping with the binding region of cyt $\mathrm{c} 2$ is shown. In all cases, 10 representative models are shown to visualize the clusters of binding models. No binding region for cyt cy on CIV could be defined since no XL was found between the proteins. 


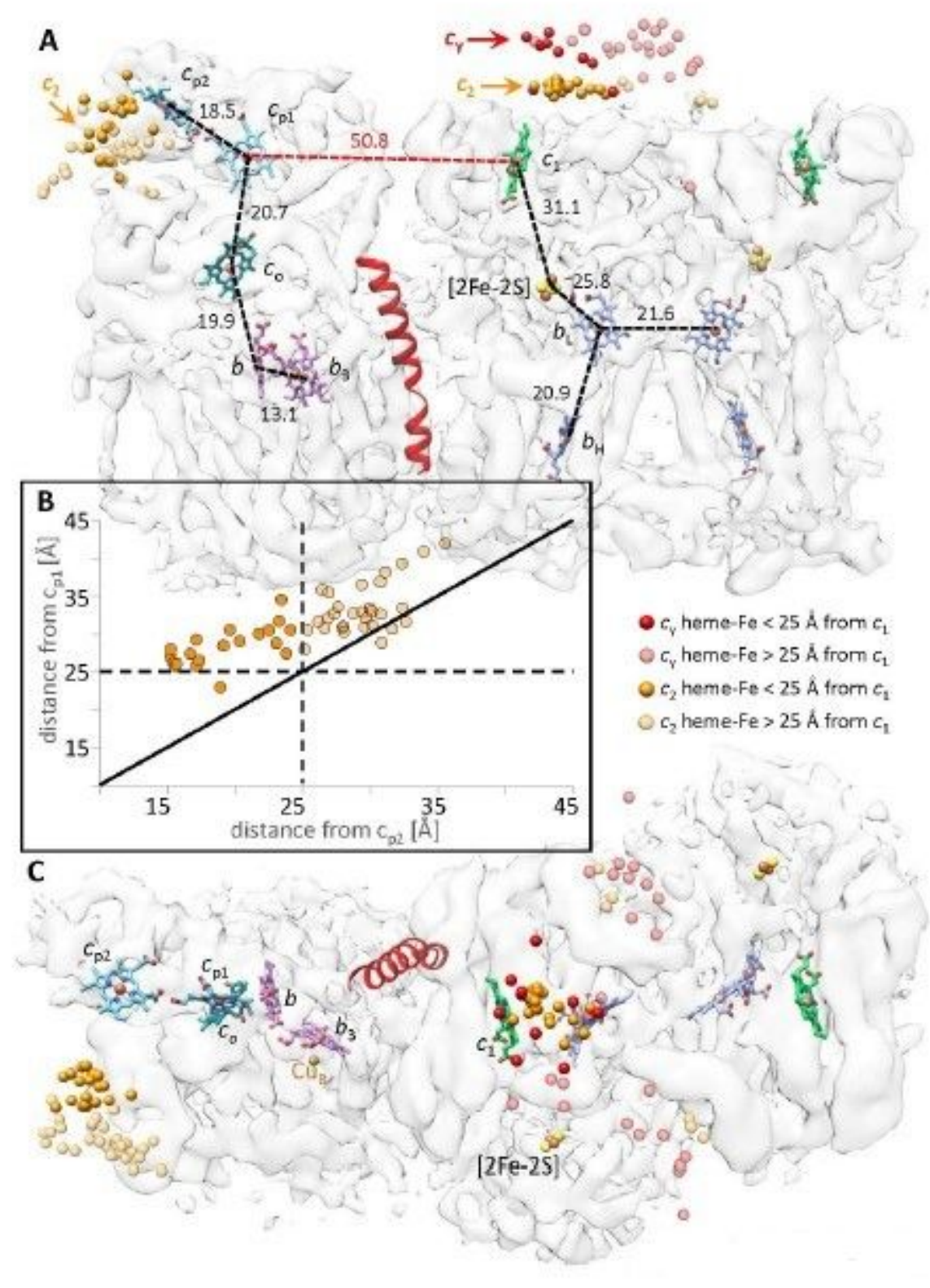

Figure 5

Organization of CIII2CIV cofactors and redox partners. A. The 1201 cofactors are shown inside the transparent map SC-2A of CIII2CIV with the same subunit colors as in Fig. 2: hemes bL and bH (periwinkle), heme $\mathrm{c} 1$ (green), hemes cp1 and cp2 (light blue), heme co (dark green), hemes b and b3 (purple). The [2Fe-2S] clusters are shown as yellow-red spheres. In all cases the distances (heme-Fe to heme-Fe) between the heme cofactors are indicated. The positions of docked cyt c2 and cyt domain of cy are indicated as orange (heme c2) and red (heme cy) spheres, respectively, representing their heme-Fe atoms. All heme-Fe atoms corresponding to the top 50 docking positions for cyt c2 on CIV are shown as solid $(<25 \AA)$ or transparent $(>25 \AA)$ spheres, depending on their distances to heme cp2. In the case of CIII2, only the docking positions of cyt $\mathrm{c} 2$ and cyt cy on monomer $A$ and between the monomers $A$ and $B$ are shown, omitting those located on monomer $\mathrm{B}$. The TMH of cyt cy is shown in red at CIII2CIV interface. B. The heme-Fe atoms of all 50 cyt c2 models docked onto CIV are plotted in function of their distances 
from heme cp1 and heme cp2, with the Fe atoms within $25 \AA$ shown as solid spheres, and those beyond $25 \AA ̊$ as transparent spheres. The vast majority of heme-Fe atoms of docked cyt c2 models are closer to heme $\mathrm{cp} 2$ than heme $\mathrm{cp} 1$ of CIV. C. Top view of the map shown in A is presented to better visualize the distribution of the docked cyt domain of cy on monomer $A$ and between the monomers $A$ and $B$. In all cases, the heme-Fe atoms are depicted by spheres and colored as indicated above and on the figure.

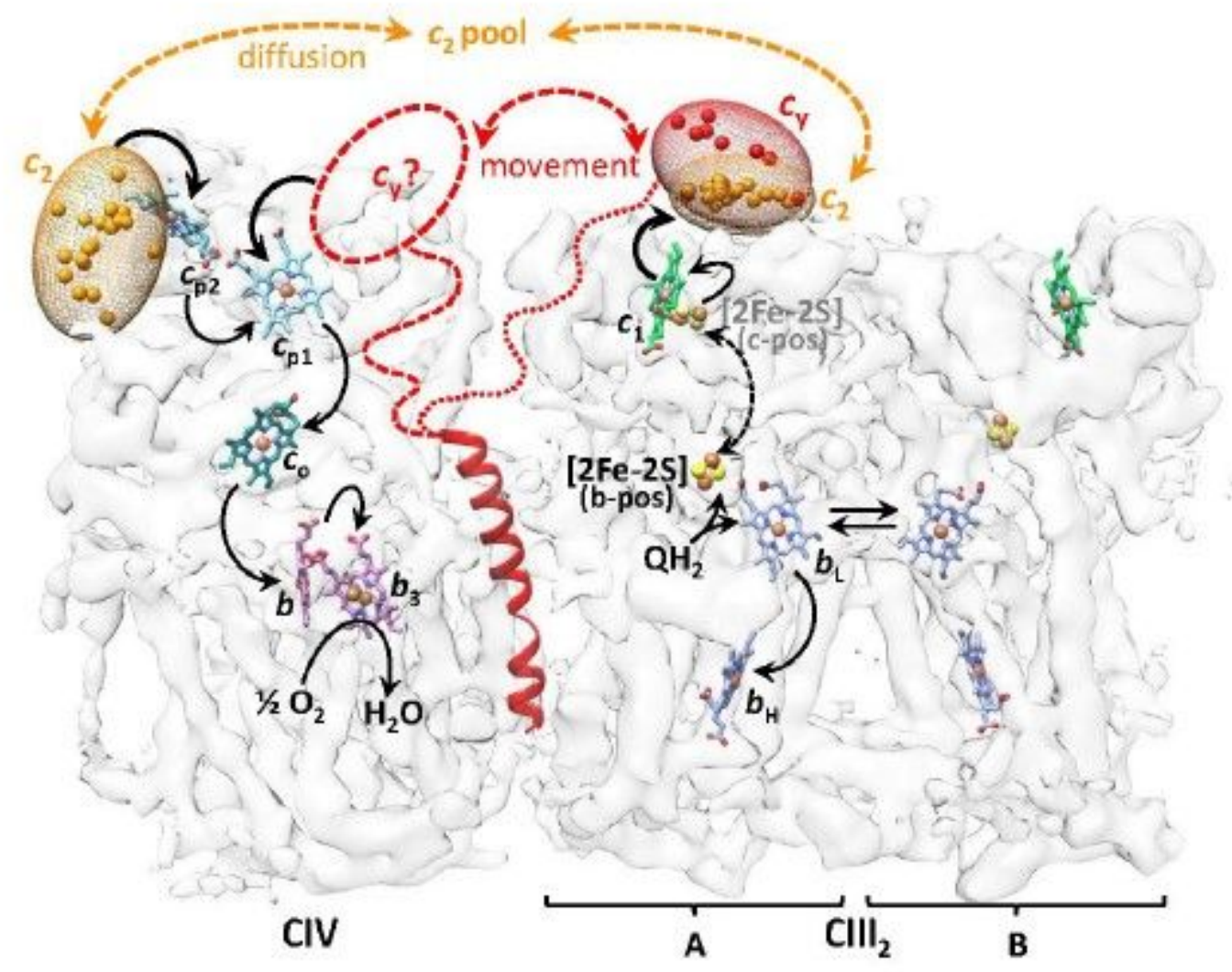

Figure 6

Proposed cyt c2 and cyt cy binding regions of CIII2CIV and electron transfer pathways. The likely 1220 binding regions of cyt $\mathrm{c} 2$ and cyt $\mathrm{c}$ domains of cy (orange and red ellipsoids, respectively), defined by XLMS guided docking, are depicted by the distributions of their heme-Fe atoms on the transparent map SC$2 \mathrm{~A}$ of CIII2CIV. Only the positions that are within $25 \AA$ of heme $\mathrm{c} 1$ of CIII2 or heme cp2 of CIV are indicated. The cofactors together with the TMH of cyt cy are shown as in Fig. 5. The linker region (indicated by dotted or dashed lines) between the TMH and the cyt domain of cy is not resolved in the cryo-EM map. The proposed electron transport pathways are shown by thicker black arrows: upon QH2 oxidation by CIII2, cyt cy which is integral to CIII2CIV receives an electron from heme c1. It then moves (double-headed dashed red arrow) to an undefined binding region (dashed oval with cy?) on CIV, where it delivers the electron to the nearest heme $\mathrm{cp} 1$ of CIV. Similarly, cyt c2 which is peripheral to CIII2CIV also receives an electron from heme $\mathrm{c} 1$, diffuses away to reach CIV and conveys it to heme cp2. The canonical electron transfers occurring from $\mathrm{QH} 2$ to heme $\mathrm{c} 1$ in $\mathrm{ClI} 2$, and from heme $\mathrm{cp} 1$ to $\mathrm{O} 2$ in $\mathrm{CIV}$, are indicated by thinner arrows. The double headed dashed black arrow depicts the movement of the [2Fe-2S] of FeS protein from the $b$ position (b-pos, black, solid spheres) to the $c$ position (c-pos, grey, transparent spheres) in CIII2 
during $\mathrm{QH} 2$ oxidation. A possible electron equilibration between the two heme bL of $\mathrm{ClI} 2$ is indicated by double arrows, and the electron transfer steps subsequent to heme bH reduction are not shown.

\section{Supplementary Files}

This is a list of supplementary files associated with this preprint. Click to download.

- SCSupplementfiguresinserted.pdf

- nrreportingsummaryDaldal.pdf

- validationreportsDaldal.zip 